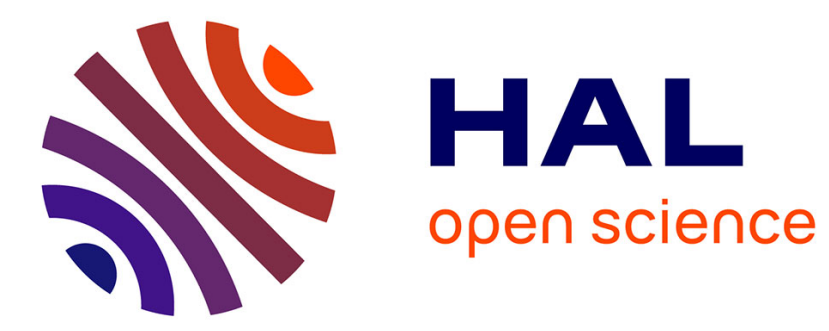

\title{
MSH Paris-Saclay, 5 ans d'interdisciplinarité sur un plateau
}

André Torre

\section{To cite this version:}

André Torre. MSH Paris-Saclay, 5 ans d'interdisciplinarité sur un plateau: Colloque du 20 novembre 2019. MSH Paris-Saclay, 5 ans d'interdisciplinarité sur un plateau, Nov 2019, Paris, France. Hors-série (5), MSH Paris-Saclay Éditions, pp.184, 2019, Actes, 978-2-490369-04-1. hal-03257118

\section{HAL Id: hal-03257118 \\ https://hal-rnmsh.archives-ouvertes.fr/hal-03257118}

Submitted on 10 Jun 2021

HAL is a multi-disciplinary open access archive for the deposit and dissemination of scientific research documents, whether they are published or not. The documents may come from teaching and research institutions in France or abroad, or from public or private research centers.
L'archive ouverte pluridisciplinaire HAL, est destinée au dépôt et à la diffusion de documents scientifiques de niveau recherche, publiés ou non, émanant des établissements d'enseignement et de recherche français ou étrangers, des laboratoires publics ou privés. 


\section{$\dot{-} \equiv \mid$ MSH PARIS-SACLAY}

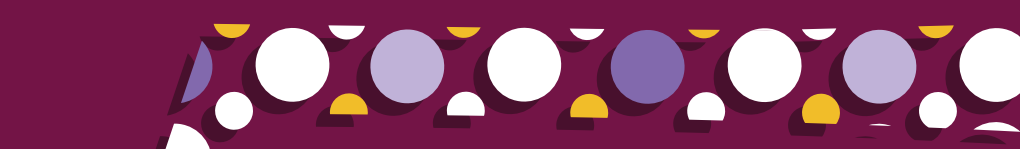

5 ANS

d'interdisciplinarité

sur un Plateau

DIRECTION : André Torre

COLLOQUE du 20 Novembre 2019 


\section{ÉDITION}

André Torre

Directeur de la MSH Paris-Saclay

\section{COORDINATION}

Éric Valdenaire

Chargé de communication, MSH Paris-Saclay

\section{SECRÉTARIAT DE RÉDACTION}

Anne-Sophie Décriaud

Éditrice, MSH Paris-Saclay

\section{ILLUSTRATIONS ET MAQUETTE}

Léa Avril

Graphiste, MSH Paris-Saclay

\section{ENTRETIENS}

Propos recueillis par Sylvain Allemand

Journaliste, rédacteur en chef de Paris-Saclay Le Média 


\section{$\stackrel{0}{-1} \begin{aligned} & \text { Maison des } \\ & \text { sciences de } \\ & \text { I'Homme } \\ & \text { paris-saclay }\end{aligned}$}

\section{MSH PARIS-SACLAY 5 ANS D'INTERDISCIPLINARITÉ SUR UN PLATEAU}


CMSH Paris-Saclay Éditions, 2019.

61 avenue du Président Wilson, 94230 Cachan www.msh-paris-saclay.fr

ISBN 978-2-490369-04-1 


\section{LA MSH PARIS-SACLAY \\ 5 ans d'interdisciplinarité sur un Plateau}

\section{SOMMAIRE}

André Torre, La MSH Paris-Saclay

Itinéraire scientifique et méthode de travail pour une ambition territoriale

9

\section{ENTRETIENS AVEC LES TROIS DIRECTEURS DE LA MSH PARIS-SACLAY}

Un « travail » d'accoucheur... Rencontre avec Claude Didry 53

Entre bottom up et top down. Rencontre avec Stefano Bosi 65

Le temps de la maturité. Rencontre avec André Torre 75

\section{PORTRAITS DE CHERCHEURS}

Antonio Casilli - Sous les plateformes numériques, le digital labor

89

Nathalie Delprat - Quand l'art et la science inventent ensemble d'autres explorations de soi

Jean-Philippe Denis - Entre économie et sociologie, les sciences de gestion voient au-delà de l'entreprise

Anaiis Fléchet - Relations internationales et globalisation culturelle au rythme de la musique

Catherine Geel - Une approche historique et comparative du design

Florent Le Bot - De l'interdisciplinarité en compagnie de Durkheim

Romain Melot - TerriBio ou comment valoriser les productions alimentaires locales

Antoine Nebout - Quand des économistes se penchent sur nos assiettes

loana Vasilescu - La linguistique à I'heure de l'IA et des systèmes automatiques 

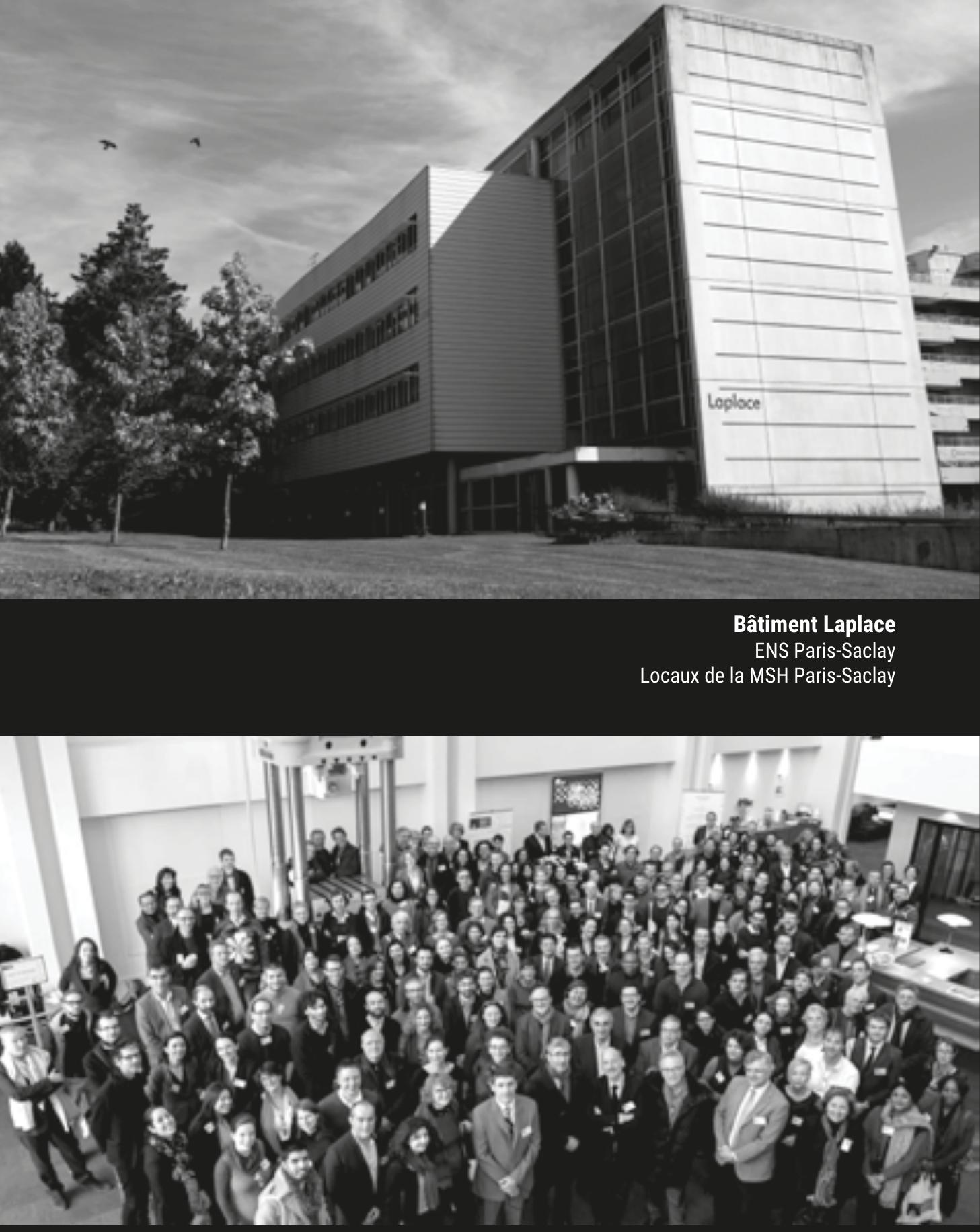

Colloque Inaugural 2017 


\section{LA MSH PARIS-SACLAY :}

ITINÉRAIRE SCIENTIFIQUE ET MÉTHODE DE TRAVAIL POUR UNE AMBITION

\section{TERRITORIALE}





\section{La MSH Paris-Saclay \\ Itinéraire scientifique et méthode de travail pour une ambition territoriale}

\section{André TORRE}

Directeur de la MSH Paris-Saclay

La construction de l'Université Paris-Saclay (UPSaclay) et son insertion dans l'ambitieux projet d'écosystème d'innovation local ont dû, dès leur origine, se confronter à un double enjeu :

- rassembler et faire travailler ensemble des scientifiques provenant d'horizons différents (disciplines variées, mais aussi universités, écoles, instituts, etc.) ;

- renforcer de manière décisive la présence d'activités scientifiques sur un territoire où coexistent d'importantes forces de recherche, des activités d'innovation et de développement industriels, et des populations locales pas toujours convaincues.

L'Université Paris-Saclay est avant tout considérée comme une entité à fort potentiel technologique. Pourtant, les SHS (Sciences de l'Homme et de la Société) ont un rôle majeur à y jouer. Tout d'abord, parce que le nombre de chercheurs et d'enseignants chercheurs dans ces disciplines s'avère très important (environ 1200 personnes), mais encore davantage 
parce que les SHS sont appelées à occuper une place essentielle dans les activités et dispositifs d'innovation et d'implantation des activités sur le plateau de Saclay.

La MSH Paris-Saclay, créée en 2015, a une histoire récente, mais animée, avec déjà trois directeurs et une croissance importante de ses effectifs et de ses actions. Elle est en mesure d'apporter sa contribution à ce défi, par son engagement au service des équipes SHS présentes dans le périmètre de Paris-Saclay, et de conforter ainsi sa place dans le Réseau national des $\mathrm{MSH}$, garant d'ouverture nationale et internationale de la recherche. Elle a également vocation, par la politique de site du CNRS, à s'intéresser à l'ensemble des recherches réalisées au sein du périmètre saclaysien, en particulier par celles des équipes SHS.

Le travail réalisé au cours de ses cinq premières années d'existence, sur la période 2015-2019, s'est avéré à la fois important et fécond. Il a placé la MSH sur de très bons rails, en lui permettant d'occuper une place centrale dans la promotion et l'organisation des recherches réalisées par les équipes SHS du site. Il a également contribué à donner une visibilité aux SHS dans l'UPSaclay, à accroître leur interconnaissance et à les fédérer au sein de collectifs de travail. La MSH a ainsi fait la preuve de sa capacité à s'insérer et à exister au sein de la communauté de recherche de Paris-Saclay en développant une position intéressante d'interface entre les SHS, mais aussi d'ouverture aux autres disciplines présentes sur le site (sciences de la vie, sciences exactes, sciences de l'ingénieur).

Au-delà de l'interaction avec les chercheurs, la MSH Paris-Saclay peut également développer les interactions avec les mondes socioéconomiques et la société civile. Elle se trouve en effet placée au cœur d'un écosystème qui implique tant de grandes entreprises que des start-ups, au centre d'un cluster scientifique et technique de haut prestige et de très grande technicité. Par ailleurs, l'existence d'une Opération d'intérêt national (OIN) sur le plateau de Saclay, avec les dimensions d'aménagement du territoire, de mobilités et de biodiversité que cela implique, indique la nécessité de se pencher sur les demandes et le bien-être des populations locales. 


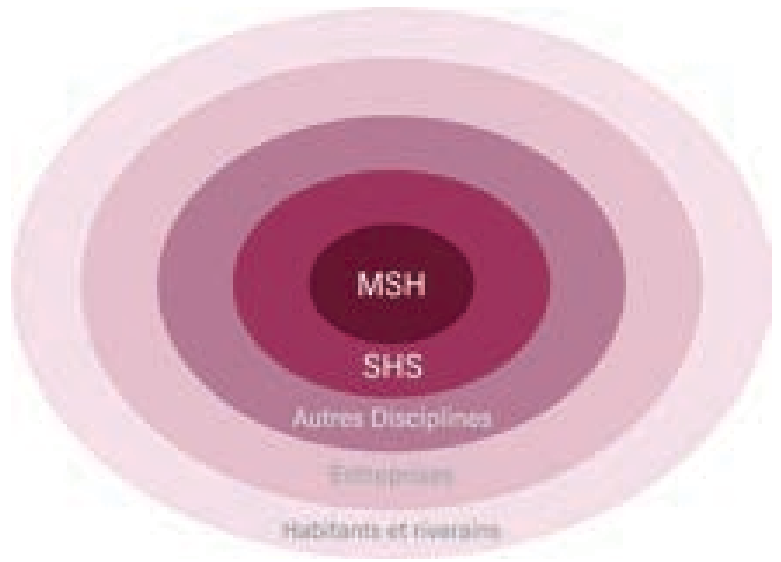

La MSH au cœur de l'écosystème Paris-Saclay

\section{Historique de la MSH Paris-Saclay}

La Maison des sciences de l'Homme (MSH) Paris-Saclay a été créée le 1er janvier 2015, sur décision de l'Institut des sciences humaines et sociales (INSHS) du CNRS, après une phase intense de concertation et de discussions, réalisée sous la responsabilité de son premier directeur : Claude Didry. L'idée de sa création, déjà ancienne, a pris corps durant les années 2010, suite à différentes initiatives visant à une première tentative de repérage et de rassemblement des SHS au sein de l'ambitieux ensemble saclaysien en constitution.

Les débuts ont été lents, en raison de l'immersion dans l'univers mouvant, et lui aussi en voie de définition, de l'Université Paris-Saclay, et plus généralement du cluster scientifique et technique du plateau de Saclay et de ses alentours. Aux enjeux du territoire s'ajoutaient l'hétérogénéité des forces en présence, ainsi que la volonté de construction d'une MSH parallèlement au département SHS de l'Université Paris-Saclay. Aujourd'hui, la MSH Paris-Saclay est devenue un point de référence pour les SHS du site et leurs interactions, ainsi qu'une actrice incontournable de l'interface avec les autres disciplines scientifiques. 


\subsection{Les prémisses : MSH et Département SHS}

Sous l'impulsion de plusieurs institutions de recherche et d'enseignement supérieur comme l'ENS Paris-Saclay, ${ }^{1}$ l'Université Paris-Sud (UPSud), l'École Polytechnique, et de différents grands organismes (CNRS, CEA, Inra, etc.), naît, dès 2011, le projet d'un recensement des forces en sciences humaines et sociales (SHS), aux contours encore incertains mais où émergent de grands groupes disciplinaires (économie, droit, sociologie en particulier). Apparaît ainsi rapidement l'idée de les identifier et de les organiser au sein d'un ensemble aux frontières épousant celles de l'Université Paris-Saclay en préfiguration.

Après une phase d'instruction, dès juillet 2013 s'impose l'idée de la création de deux structures aux fonctions différentes mais complémentaires : le Département de sciences sociales et la MSH - le premier ayant vocation à représenter l'Université Paris-Saclay (UPSaclay) dans sa composante SHS, alors que la seconde doit incarner l'intérêt manifesté par le CNRS pour l'opération inédite en cours sur le plateau et pour l'interface avec les disciplines des sciences techniques, du vivant et de lingénieur. Toutes deux ont la prétention de réunir l'ensemble des SHS de Paris-Saclay sans exclusive, mais leurs missions et domaines d'actions respectifs demandent à être définis.

La tâche initiale, confiée à Claude Didry, alors Directeur de recherche au CNRS au sein de l'ENS Paris-Saclay, comporte la mise en préfiguration des deux structures, assortie d'une réflexion sur leurs rôles respectifs. À travers les réunions de l'Assemblée des directeurs d'unité et suivant les injonctions de la COMUE Paris-Saclay se dessinent alors les complémentarités et synergies à créer. D'une part, un département SHS centré sur des prérogatives disciplinaires et ayant pour vocation à rassembler et donner vie et identité aux différents groupes de disciplines des SHS, sans oublier les questions d'enseignement. D'autre part, la $\mathrm{MSH}$, laboratoire du CNRS, qui doit se consacrer aux problématiques de recherche et a vocation à favoriser l'interdisciplinarité, entre les différentes disciplines des SHS mais également vers les autres disciplines scientifiques présentes dans l'ensemble saclaysien.

Anciennement ENS Cachan. 


\subsection{Le projet AERES}

Le projet de demande de création d'une MSH sous statut d'USR (Unité de services et de recherche) est déposé par l'ENS Paris-Saclay à l'automne 2013, sous l'intitulé : « Maison des sciences de l'Homme Paris-Saclay », avec deux tutelles : le CNRS et l'UPSaclay, pas encore créée à ce moment.

La structure définie dans cette proposition initiale se propose de fédérer les compétences en sciences de l'Homme et de la Société présentes dans le périmètre Paris-Saclay. Elle entend également lier les sciences de l'Homme et les sciences sur l'Homme, et mettre en place des interfaces et des synergies entre ces deux blocs disciplinaires, en mobilisant le potentiel d'expertise et de réflexivité des sciences de la nature regroupées dans ce territoire depuis plusieurs décennies. La MSH Paris-Saclay vise ainsi à contribuer à la promotion d'un humanisme scientifique et technologique autour de cinq axes majeurs, correspondant aux thématiques suivantes :

- la constitution de bases de données scientifiques et la modélisation ;

- les relations entre recherche, entreprise et travail ;

- l'environnement, le développement territorial et les mobilités ;

- les cultures et les patrimoines, avec une dimension historique et artistique ;

- le droit, la régulation et les dynamiques institutionnelles.

Son activité principale doit consister à stimuler la transversalité des recherches à travers l'élaboration d'un appel à projets annuel financé par ses ressources récurrentes. Elle a également vocation à proposer un appui aux projets répondant à des programmes européens, ANR ou autre grand programme, ainsi qu'à accueillir des séminaires communs et des manifestations scientifiques. Sont également évoquées la création d'un pôle documentaire d'excellence, intégrant l'accès à des équipements de pointe, ainsi que celle d'un pôle d'édition fédérant la réalisation des revues portées par les laboratoires, avec une ouverture sur l'édition numérique. Il est enfin proposé que la cellule de préfiguration soit abritée à Cachan par l'ENS Paris-Saclay, avant l'affectation d'un bâtiment répondant à ses besoins. 
Ce dossier, qui présente le premier effort important de définition des grandes thématiques des SHS de Paris-Saclay et de leur organisation en fonction d'axes cohérents, est une ébauche, marquée par la dynamique et le volontarisme d'un certain nombre de personnes et d'institutions, au premier chef desquelles figure son futur directeur. Le projet reçoit un avis favorable du CNRS, qui décide de la création de la MSH ParisSaclay. La préfiguration de l'UPSaclay, la mise en place d'une OIN, la volonté de concentration de forces de recherche dans un environnement favorable à l'innovation et au partage des connaissances ont pesé en faveur du lancement d'une structure susceptible de fédérer et de promouvoir les forces de recherche en sciences de l'Homme et de la Société.

\subsection{La mise en place de l'unité - Acte 1}

L'année 2015 est celle du démarrage institutionnel de la MSH ParisSaclay. Elle est surtout consacrée à une réflexion sur les modes de structuration et d'organisation internes, ainsi qu'à un repérage plus précis des forces en présence en SHS. L'organisation repose sur quatre tutelles principales - CNRS, ENS Paris-Saclay, Université Paris-Sud (UPSud) et Université Versailles Saint-Quentin-en-Yvelines (UVSQ) -, le reste des intervenants de Paris-Saclay participant en tant que "partenaires » (CEA, HEC, ENSAE, ENSTA, Mines ParisTech, École Polytechnique, AgroparisTech, Institut d'Optique Graduate School (IOGS), Inra, Télécom Sud Paris, Télécom ParisTech, Université d'Évry-Val-d'Essonne (UEVE), CentraleSupélec et FCS Campus Paris-Saclay). Différentes possibilités d'installation dans des locaux propres sont envisagées, avant que le choix ne se porte sur la solution de l'hébergement dans les locaux actuels de l'ENS Paris-Saclay, ainsi que son intégration future dans ses nouveaux bâtiments, en construction sur le plateau de Saclay.

Un groupe de préfiguration des structures SHS est désigné par l'Assemblée des directeurs d'unités ${ }^{2}$, avec pour mission de définir les futurs équilibres et de donner vie et sens aux recherches, ainsi que de procéder au premier recensement des chercheurs et enseignants-chercheurs du

2 Ce groupe était composé de Claude Didry, Hélène Aubry, Hélène Gispert, Stefano Bosi, Hervé Dumez, André Torre et Laurent Willemez. 
domaine. L'enquête révèle que leur nombre avoisine les 1 200, rendant ainsi objective la nécessité de structurer et d'organiser cette communauté. Sans doute plus importante qu'on ne l'imaginait, elle apparait également très morcelée et fragmentée, $y$ compris géographiquement. Les recherches s'effectuent parfois dans des laboratoires de petite taille, relativement isolés, et les chercheurs ne se connaissent pas encore pour l'essentiel.

Au cours de l'année 2015, les tâches et fonctions se précisent. Laurent Willemez, Professeur à l'UVSQ, prend la charge du Département SHS, dont il va devenir le premier directeur en 2016. Du côté de la MSH, Stefano Bosi, Professeur à l'UEVE, et André Torre, Directeur de recherche à l'Inra, deviennent Directeurs adjoints et contribuent à la réflexion sur le développement de la structure et de ses axes de travail, ainsi qu'aux modalités de mise en ouvre des appels à projets et des workshops. À l'automne 2015 est recrutée la Secrétaire générale, appelée à prendre ses fonctions en janvier 2016.

Le premier semestre 2016 voit la MSH Paris-Saclay trouver sa place au sein des locaux de l'ENS Paris-Saclay. Plusieurs réunions avec la présidence de la COMUE et la totalité des tutelles permettent de poser les besoins et les modalités de gestion d'un financement suffisant pour proposer un programme d'activités.

\subsection{La mise en place de l'unité - Acte 2}

En septembre 2016, Stefano Bosi est nommé Directeur de la MSH Paris-Saclay. À sa prise de fonction, il s'entoure d'un bureau ${ }^{3}$ de six personnes, représentant pour parties les tutelles de l'unité mais aussi la diversité des disciplines, et s'attache à finaliser la mise en place du Conseil scientifique, ainsi que la programmation d'appels à projets et d'événements scientifiques.

Un nouveau projet de recherche est défini, s'articulant autour de quatre grands axes, respectivement intitulés :

3 La composition du bureau était la suivante : Sandra Charreire-Petit (UPSud), Éric Godelier (École Polytechnique), Stéphanie Lacour (ENS Paris-Saclay/ CNRS), André Torre (Inra-AgroParisTech), Laurent Willemez en qualité de Directeur du département SHS (UVSQ) et Célia Zolynski (UVSQ). 
- le Pouvoir des algorithmes, autour des thématiques Big Data, réseaux, théorie de la décision, modélisation ;

- la Culture de la nature, autour des thématiques de transition énergétique, climat, biodiversité, ville, usage des sols, alimentation ;

- la Nature de la culture, autour des questions culturelles et patrimoniales, artistiques et historiques ;

- la Santé de demain, autour des nouvelles technologies (biotechnologies et e-santé), des styles de vie, des systèmes de santé et des questions éthiques.

Sont également proposés deux axes transversaux :

- la Mondialisation (mal) heureuse, sur la mondialisation économique et culturelle ;

- l'Innovation permanente, s'articulant avec les sciences et techniques dans le contexte du cluster de l'Université Paris-Saclay.

Afin de proposer des services aux chercheurs et aux équipes du site deux leviers de programmation scientifique sont mobilisés pour 2016 : un appel à projets, autour de thématiques interdisciplinaires et interinstitutionnelles, ainsi que la labélisation d'événements scientifiques "au coup par coup".

La composition du Conseil scientifique est finalisée fin 2016. Il comprend 24 membres, dont 8 étrangers, et s'efforce de représenter une pluralité de disciplines des SHS, mais aussi des sciences du vivant. Réuni pour la première fois la veille du Colloque inaugural de la MSH ParisSaclay, qui rassemble, le 27 janvier 2017, 250 participants autour d'une réflexion sur ses axes, à Saint-Rémy-lès-Chevreuse (78), il recommande une réduction du nombre d'axes scientifiques et un resserrement des thématiques autour de domaines ciblés et prioritaires. Il en résulte une reformulation en trois grands axes de recherches, actuellement utilisée $e^{4}$.

Sous la direction de Stefano Bosi, une programmation scientifique est proposée à la communauté SHS de Paris-Saclay en 2017. Elle repose sur deux

4 Voir leur présentation infra. 
types d'appels à projets (appels à projets «Émergence » et « Maturation ») et deux types d'appels à événements (appels à projets « Workshops » et «Séminaires »), avec deux ou trois vagues de retours de dossiers sur l'année, ce qui permet une veille permanente sur les activités de recherche et une réactivité forte aux propositions des collectifs de chercheurs.

$\mathrm{Au}$ total, plus de 60 événements sont labellisés et/ou organisés entre 2016 et 2017. Ils permettent un début de structuration de la communauté des SHS, ainsi que sa reconnaissance et sa visibilité à l'égard des autres disciplines de l'UPSaclay, et au-delà, sur le périmètre saclaysien.

C'est avant tout la mise en place d'une équipe de personnels qui a permis la réalisation de ce programme. En effet, quatre collaborateurs « permanents » ont rejoint la Secrétaire générale :

- un Gestionnaire administratif et financier, en juin 2016 ;

- une Éditrice (secrétaire de rédaction), en mars avril 2017 ;

- un Chargé de communication, en juin 2017 ;

- un Responsable de la coordination scientifique, en juillet 2017.

L'arrivée de ces collaborateurs a contribué, étape par étape, à proposer de nouveaux services, qu'il s'agisse de la gestion administrative et financière des projets, de la communication sur les événements scientifiques, de l'accompagnement des chercheurs du périmètre dans l'identification des ressources et le montage de projets, ou de l'accueil de revues scientifiques, etc.

\subsection{La consolidation et le décollage}

En mars 2018, Stefano Bosi est nommé Vice-Chancelier des Universités de Paris et doit donc abandonner sa position à la tête de la MSH. Suite à un concours organisé par le CNRS avec l'appui des autres tutelles, André Torre, Directeur de recherches à l'Inra et rattaché à AgroParisTech, est nommé Directeur de la MSH en mai 2018, sur la base de son programme de développement des activités de recherche. Il inscrit résolument son action dans la continuité de celle de ses prédécesseurs et s'entoure d'un bureau renouvelé et élargi ${ }^{5}$.

5 Le nouveau bureau est composé de Sandra Charreire-Petit (UPSud), 
La première tâche est celle de la rédaction du rapport et de la préparation des éléments nécessaires à la visite du Comité du HCÉRES (Haut Conseil de l'évaluation de la recherche et de l'enseignement supérieur), qui doit évaluer le bilan des opérations réalisées depuis la création de la structure. Le Comité de visite, qui se déroule en novembre 2018 sous la présidence de Serge Wolikow (historien, Université de Bourgogne), pointe les grands progrès réalisés par la MSH Paris-Saclay. Il lui accorde un satisfecit général quant à son action, ainsi qu'une appréciation extrêmement positive sur les opérations réalisées et sur le projet de développement de ses activités.

L'opinion générale qui ressort du rapport du Comité est très favorable : "On ne peut qu'être impressionné favorablement par le travail de structuration réalisé par la direction de la MSH Paris-Saclay qui a réussi à associer, selon des modalités différenciées et efficaces, séminaires, rencontres, ateliers, journées d'études, le plus grand nombre

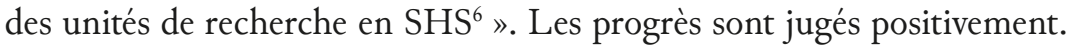
"Ces développements ont reposé sur l'activité de la direction épaulée par un conseil scientifique étoffé ». La gouvernance d'ensemble est confortée. «L'implication forte des tutelles est un élément essentiel pour permettre à la MSH Paris-Saclay de consolider son ancrage et d'engager une nouvelle étape de son évolution, nonobstant les incertitudes qui affectent encore l'ensemble du site. »

Suite au départ inattendu de la Secrétaire générale, l'équipe se resserre alors autour de ses objectifs et de quelques grandes opérations de développement :

- mettre en place et lancer l'annuaire des chercheurs en SHS ;

- définir et formaliser une méthode de travail pour la MSH et ses activités ;

Mélanie Clément-Fontaine (UVSQ), Éric Godelier (École Polytechnique), Stéphanie Lacour (ENS Paris-Saclay/CNRS), Jean-Paul Markus en qualité de Directeur du département SHS (UVSQ), François Sarfati (Université d'Évry) et Paola Tubaro (ENS/CNRS).

6 Évaluation de la structure fédérative Maison des sciences de l'Homme ParisSaclay, HCÉRES, mars 2019. 
- poursuivre et développer les activités liées aux différents types d'appels à projets ;

- modifier et améliorer les procédures des appels ;

- mettre en place une nouvelle catégorie d'appel à projets de recherche, plus ambitieuse.

L'annuaire des chercheurs est mis en place en décembre 2018, suite à une campagne de recensement auprès de l'ensemble des chercheurs SHS Paris-Saclay. Près de 500 chercheurs y figurent désormais, après avoir défini leurs objets et modes de recherche. Cet outil en accès libre permet à tous les chercheurs du périmètre de trouver, via un moteur de recherche multi-critères, des partenaires pour monter des projets interdisciplinaires et répondre ainsi plus facilement aux appels à projets de la MSH Paris-Saclay.

La méthode de travail mise en place vise à rationaliser les différentes opérations menées, à inscrire chaque projet dans une logique d'ensemble et à rendre l'activité de la MSH Paris-Saclay lisible à l'extérieur. Elle repose sur l'idée que l'exigence scientifique peut se réaliser par la mise en œuvre d'une politique dédiée, associant des démarches d'appels à projets de type bottom-up et top-down, au service des recherches menées entre différentes disciplines des SHS et au-delà.

\begin{tabular}{|c|c|c|}
\hline & Interactions SHS & $\begin{array}{c}\text { Interface disciplines } \\
\text { hors SHS }\end{array}$ \\
\hline Bottom-up & $\mathrm{X}$ & \\
\hline Top-down & & $\mathrm{X}$ \\
\hline
\end{tabular}

Pour les recherches en SHS, dont les sollicitations sont avant tout de type bottom-up, il s'agit surtout de poursuivre et d'améliorer l'effort entrepris, qui a procuré une large satisfaction au collectif des chercheurs en SHS. 
Les appels à projets, cœur de l'activité de la MSH Paris-Saclay, subissent différentes améliorations ou corrections. Lancés dans l'urgence et de manière exploratoire, ils ont connu un grand succès. Le moment est venu d'en faire un bilan et de tirer des enseignements des premières années de fonctionnement afin de les améliorer et de les rendre encore plus efficaces et adaptés aux besoins de leurs utilisateurs.

L'ensemble du système est revu et amélioré. Après évaluation, il est décidé une mise en cadence des appels, au cours des trois campagnes annuelles, un affinement des critères (en particulier le refus absolu des projets monodisciplinaires ou mono-institutionnels), la mise en place de nouveaux critères budgétaires et de dates de clôture, ainsi qu'une modification de la procédure d'évaluation des projets "Émergences », séminaires et workshops. Est ainsi constitué un groupe d'experts disciplinaires de la MSH Paris-Saclay, issus des anciens porteurs de projets labellisés. Les projets courants sont désormais évalués conjointement par un de ces experts disciplinaires et un membre du bureau de la $\mathrm{MSH}$ Paris-Saclay. Par ailleurs, sont créées des Journées de restitution des projets "Émergence » et «Maturation », qui permettent à la fois de revenir sur le travail réalisé et de le populariser auprès de la communauté des chercheurs SHS du site par un rendu public et médiatisé.

En ce qui concerne les relations avec les disciplines hors SHS, une action plus volontariste est décidée, avec un financement adéquat et une logique davantage top-down. Il s'agit de la mise en place d'un nouvel appel à projets de recherche, intitulés " Excellence ». L'objectif est de donner naissance et de financer une famille de projets plus ambitieux, de taille plus importante, et axés sur l'interdisciplinarité élargie en dehors des SHS. Si chaque projet est avant tout SHS, il doit impliquer également des disciplines autres et faire l'objet d'un co-portage entre des chercheurs des deux univers. Il s'agit d'identifier des équipes susceptibles d'élaborer des réponses à des projets internationaux type H2020 et de les accompagner dans cette démarche, par des soutiens adaptés. Les thématiques proposées visent à créer ou identifier des groupes d'excellence interdisciplinaires, pilotés par des équipes SHS, au sein de l'UPSaclay.

Lancé en décembre 2018, cet appel rencontre à son tour un grand succès avec de nombreux dépôts de candidature. Après examen par des 
experts extérieurs et classement par le Bureau, il fait l'objet d'un choix final par le Conseil Scientifique en mars 2019, qui désigne un lauréat, le projet TerriBio, à l'interface des SHS et des sciences du vivant.

À cette occasion, le Conseil scientifique, qui termine son mandat, adresse à la MSH Paris-Saclay et à son équipe les plus chaleureuses félicitations et donne des conseils pour les futurs développements des activités.

«Le Conseil scientifique (CS) tient tout d'abord à féliciter l'équipe de la MSH et son directeur pour le travail réalisé pendant l'année écoulée. La quantité d'activités soutenues et accompagnées par la MSH est toujours aussi élevée. Par ailleurs, le travail de maillage et d'inscription sur le territoire des activités de la MSH s'est poursuivi notamment avec des rencontres dans presque chacun des 37 laboratoires de SHS du site, et la constitution d'un annuaire en ligne des chercheurs. La nouvelle maison d'édition a publié ses premiers actes de colloques. Comme le souligne le rapport du HCÉRES, le bilan est très positif. ${ }^{7}$

Ainsi se clôt une période de cinq ans de travail intense. Aujourd'hui, la MSH Paris-Saclay est en très bon état de marche et présente des réalisations nombreuses et parfois déterminantes, après des années marquées par une activité exponentielle et une reconnaissance accrue. Son dynamisme repose sur une structuration des appuis à la recherche en fonction de trois grands axes clairement définis, sur des appels à projets et à événements fructueux et générant un dynamisme reconnu, ainsi que sur des services de qualité.

\section{Les axes de recherche}

La MSH Paris-Saclay a vocation à impulser et soutenir les recherches entre les équipes et les disciplines des SHS, ainsi qu'avec les autres disciplines présentes sur le site. Elle affiche, en adéquation avec le projet de l'Université Paris-Saclay et la constitution du cluster saclaysien, une préférence pour certaines thématiques, qui se reflètent dans ses trois axes de recherche.

7 Extrait de l'avis final du Conseil scientifique, avril 2019. 


\section{Axe 1 : Numérique et humanités}

L’axe « Numérique et humanités » catalyse les dynamiques de recherche autour de la révolution numérique et de ses ambivalences. Si cette révolution change le visage des sociétés et des économies et représente ainsi une opportunité indéniable de développement, elle pourrait également constituer une menace pour nos libertés individuelles. Les enjeux sociétaux des réseaux, des données massives et de l'algorithmique sont ainsi au cœur des projets de recherche labellisés par la MSH Paris-Saclay.

Le monde des données est traversé par des transitions et des changements profonds. Les données qualitatives et quantitatives accumulées par les enquêtes en SHS constituent maintenant un stock considérable, destiné à s'accroitre encore dans les années à venir. On voit émerger progressivement le problème des données massives (Big Data), connectées (réseaux sociaux) ou complexes (langage naturel et images), de leur stockage (compression, indexation, streaming), de leur fiabilité et de leur traçabilité.

Mais les données massives seraient inertes sans l'usage des algorithmes de fouille. Les individus laissent quotidiennement des traces repérables dans leurs relations avec les organisations (administrations, entreprises, etc.) ou via leurs usages des technologies numériques. Ces grandes masses de données numériques, précisément datées (time-stamped) et souvent géolocalisées, attirent les entreprises et la statistique publique, mais leur traitement constitue un défi théorique et questionne les théories de la décision et de l'optimisation. Au croisement des statistiques, de l'informatique et de l'intelligence artificielle, les algorithmes doivent faciliter la construction de modèles sur les comportements et les représentations collectives ou la prise de décision, à partir du brassage de grandes masses de données, car les modèles statistiques et les algorithmes de décision traditionnels se révèlent inadaptés à traiter des données hétérogènes, complexes, incomplètes et incertaines.

Ainsi, de nouvelles recherches s'avèrent nécessaires sur les usages de la technologie et ses conséquences sur les comportements individuels et collectifs, ainsi que sur les dynamiques sociétales qui leur sont liées. Les enjeux éthiques et juridiques obligent les SHS à réfléchir à la préservation 
du patrimoine informationnel, à la protection de la vie privée, au design et à l'implémentation des régulations. D'un point de vue éthique et épistémologique, la gestion des données doit également intégrer de nouvelles contraintes telles que la sécurité et la traçabilité. La question d'une intelligence artificielle, qui surpasse l'intelligence humaine dans certaines applications, interroge ainsi directement les SHS.

Mais la révolution numérique impacte également les humanités et les activités liées à la mémoire, au patrimoine, à leur valorisation et à leur transmission. Les nouveaux moyens de calcul et de communication ouvrent ainsi de nouvelles perspectives de développement et vont rapidement bouleverser la façon de penser, d'enseigner et de diffuser les savoirs, les objets et les méthodes de recherche, grâce au mariage de l'informatique, des arts, des lettres et des SHS. Ces nouvelles humanités numériques constituent un domaine qui mérite un intérêt particulier. D'une part, parce que ces supports et méthodes vont probablement être à l'origine d'innovations dans les méthodes de recherche, d'enseignement et de diffusion de l'information scientifique et technique. Il convient, d'autre part, d'en évaluer la portée et les modalités du point de vue méthodologique et épistémologique.

Les nouvelles technologies révolutionnent également les recherches sur le patrimoine. L'économie de l'immatériel façonne à la fois le processus de patrimonialisation, les modalités de transmission et les pratiques de création culturelle. Le numérique fait émerger de nouvelles catégories, comme le patrimoine numérique, qui regroupe un ensemble de ressources et de données culturelles et patrimoniales, et impacte la façon de décrire, penser, nommer et visualiser le patrimoine. Mais la virtualisation des patrimoines matériel et immatériel ne doit pas se substituer à la conservation du patrimoine physique et des supports traditionnels. Une dimension importante de la recherche saclaysienne est ainsi liée à l'étude des matériaux et aux processus de patrimonialisation (objets de musée, collections d'histoire naturelle et d'archéologie, architecture, manuscrits et livres, archives).

Environ 400 chercheurs et doctorants SHS du périmètre travaillent sur les données, les algorithmes, la décision, et 200 d'entre eux s'intéressent à la culture et au patrimoine. À l'avenir, les deux communautés sont 
appelées à accentuer leurs collaborations, d'autant plus que le site de Paris-Saclay est très présent au niveau international dans le domaine des STIC et l'étude des matériaux anciens (imagerie, spectroscopie, analyse et modélisation). Aussi, l'Institut Convergence DATAIA (Data Science, Artificial Intelligence and Society) porte une recherche pluridisciplinaire sur les données et les algorithmes, en articulation parfaite avec cet axe.

\section{Axe 2 : Environnement et santé}

L'axe «Environnement et santé » a pour objectif de porter les interactions entre les recherches sur l'environnement, le territoire et la santé. L'UPSaclay regroupe un potentiel unique en France de recherche sur les questions environnementales, qu'il s'agisse des écosystèmes, de l'énergie, du climat ou de la biosphère. Par ailleurs, avec la concentration d'entreprises, d'hôpitaux et de laboratoires, le site de la nouvelle Université, combiné aux enjeux d'association des populations de cette grande opération d'aménagement de l'espace, ouvre des possibilités majeures en matière de recherche sur les dimensions territoriales, ainsi que sur les problématiques de santé humaine. La MSH Paris-Saclay mobilise les forces SHS du périmètre sur ces questions épineuses.

$\mathrm{Au}$ cœur des préoccupations citoyennes et politiques se trouve la question de la transition écologique, dont la composante énergétique représente probablement l'enjeu principal, tant pour la création d'emplois que la gestion des risques, l'environnement et l'engagement vers une réduction drastique des émissions de gaz à effet de serre, dans le cadre de la préservation du climat. La MSH Paris-Saclay se mobilise afin de constituer en France la première communauté SHS structurée à même de saisir les enjeux énergétiques du $\mathrm{XxI}^{\mathrm{e}}$ siècle selon des axes spatiaux (territoriaux, nationaux, planétaires) et temporels (moyen et long terme). Il s'agit aussi d'irriguer au-delà, à l'interface avec les recherches menées dans d'autres domaines de recherches (santé, écologie, climatologie, transports).

La préservation du capital naturel que représentent des écosystèmes terrestres et marins et des sols en bon état est un enjeu majeur pour les grands équilibres biophysiques, pour le bien-être des humains, mais aussi pour les services productifs et les activités socioéconomiques liées. 
Le plateau de Saclay est porteur d'une ambition qui fait écho à ces questionnements et porte les espoirs d'un regroupement de compétences scientifiques de grande importance, en termes de cluster ou d'écosystème industriel et de recherche. Il interroge l'impact des activités humaines et des processus d'urbanisation (transports, nouvelles mobilités, etc.) sur le vivant, mais aussi l'implication des populations locales dans leurs évolutions futures.

La question environnementale est ainsi indissociable de celle des territoires, dans l'agenda des décideurs publics comme dans les stratégies des entreprises et organisations, avec les problématiques d'aménagement du territoire, de réforme des collectivités, de mise en réseau des acteurs de la production et de la gouvernance. Les recherches menées au sein de la MSH Paris-Saclay portent sur trois domaines principaux de recherche :

- l'urbain, le périurbain et la nature, et la recomposition de leurs frontières géographiques et sociales en relation avec la taille des agglomérations franciliennes et l'intense étalement urbain ;

- l'aménagement et la gestion de ces espaces, qui renvoient aux approches de politiques publiques et de gouvernance des territoires ;

- les problématiques entrepreneuriales, de gestion des entreprises ou de mise en ouvre des processus d'innovation, de création et de valorisation des connaissances territoriales.

La question alimentaire est étroitement liée à celle de l'usage des sols. Les fonctionnalités aujourd'hui demandées aux produits alimentaires sont multiples, en termes de capacités nutritives, de qualités organoleptiques ou d'impact sanitaire et environnemental.

Quatre thèmes de recherche sont d'ores et déjà investis par les équipes SHS liées à la MSH :

- l'analyse des comportements alimentaires, de leurs déterminants et leurs conséquences en termes de consommation, d'effets sur la santé, d'inégalités sociales et d'impact environnemental ;

- les analyses des stratégies agricoles, industrielles et des stratégies de distribution qui définissent les caractéristiques des aliments et déterminent la localisation des activités et des commerces ; 
- l'analyse de la gouvernance de l'approvisionnement, notamment l'organisation de la diversité des filières, de l'usage des sols, de la restauration collective, ainsi que l'influence des réglementations ;

- l'évaluation des politiques publiques sur l'alimentation, avec leurs impacts sur la santé, l'environnement, ou la sécurité des approvisionnements.

Enfin, l'environnement et l'alimentation affectent également la santé humaine, avec la contribution de la pollution au développement des maladies dégénératives, l'antibiorésistance, la tropicalisation des zones tempérées ou l'éclosion de nouvelles épidémies globalisées. Des projets en SHS se penchent sur l'articulation environnement-santé, en étroite coordination avec la MSH Paris-Saclay. Des révolutions technologiques comme l'e-santé et les biotechnologies peuvent aider à la réorganisation des systèmes de santé et à surmonter les futures crises sanitaires. Elles peuvent également contribuer à mieux répondre aux défis du vieillissement et de l'allongement de l'espérance de vie (en bonne santé). De nombreuses questions éthiques et sociétales apparaissent ainsi dans le champ de la génomique, facilitées par l'utilisation des techniques de séquençage du génome et des analyses bio-informatiques. Les systèmes de santé sont appelés à s'adapter aux nouvelles crises sanitaires en se réorganisant et en profitant des avancées des nouvelles technologies (notamment numériques), alors que leur management et les modèles économiques sous-jacents sont destinés à faciliter les transitions sanitaires à venir.

Plus de 300 chercheurs et doctorants SHS du périmètre Paris-Saclay travaillent sur l'environnement et le territoire (énergie, biodiversité, espace) et une centaine sur la santé (e-santé, biotechnologies, systèmes de santé). Ces deux communautés interagissent régulièrement par le biais de projets interdisciplinaires. La thématique de l'usage des terres en relation au changement climatique portée par l'Institut Convergence CLand s'inscrit parfaitement dans cet axe. 


\section{Axe $T$ : Transition et innovation}

Les deux axes cardinaux de la MSH Paris-Saclay concernent essentiellement les transitions numérique et écologique, qui s'accompagnent le plus souvent d'innovations technologiques ou sociales susceptibles de déstabiliser les systèmes dans lesquels elles s'insèrent et de les engager dans de nouvelles transitions. Le progrès technique peut ainsi accélérer la prédation des ressources naturelles, la perte de biodiversité, le changement climatique, la perte de propriété des données personnelles, mais également proposer des solutions à ces problèmes. La nécessité de comprendre la complexité des boucles de rétroactions et la permanence des injonctions d'innover, ainsi que la présence au sein de Paris-Saclay de forces spécialisées dans la recherche sur l'innovation ont incité à définir un axe transversal, qui trouve des terrains d'applications dans toutes les thématiques étudiées au sein de la MSH Paris-Saclay.

L'innovation est en effet un objet d'étude interdisciplinaire, à la lisière des sciences de la nature et des SHS. Elle est aussi un champ de recherche pluridisciplinaire au sein de ces dernières, au croisement de l'histoire, du droit, de la sociologie, de l'économie et de la gestion. À cet égard, elle se situe au centre de nombreuses réflexions sur la propriété intellectuelle, l'entreprise et la croissance économique. Ces thématiques constituent un objet d'étude particulièrement stimulant pour toutes les disciplines des SHS. Au cœur d'un territoire qui fait de l'innovation l'un de ses fers de lance, la MSH Paris-Saclay a vocation à porter une recherche méthodologique et réflexive interdisciplinaire sur cet objet, en mobilisant non seulement les quelques 100 spécialistes du domaine au sein de ses laboratoires, mais aussi toutes celles et ceux qui souhaitent interroger la portée des injonctions d'innover sur leurs pratiques et leurs résultats.

Étudier le concept d'innovation en tant que tel, dans ses dimensions managériales, économiques ou même sociologiques ou juridiques, est un objectif, de même que l'exploration des apports des Science and Technology Studies à la compréhension des effets des politiques qui lui sont consacrées. Enjeu politique et économique, l'objet «innovation » constitue un concept-valise présenté comme étant susceptible de résoudre tous les problèmes de la société : progrès scientifique et technologique 
d'une part, changements organisationnels et institutionnels d'autre part. L'interroger, c'est questionner la pertinence des frontières entre recherche fondamentale, recherche appliquée et $\mathrm{R} \& \mathrm{D}$, de même que les critères et les outils de l'action publique et privée consacrés au financement de la recherche et à son développement.

L'étude des effets de l'injonction d'innover sur la production des connaissances scientifiques et techniques, tout comme sur nos sociétés en général, fait l'objet d'un nombre important de travaux depuis les années 1960. Ils mettent en avant l'idée que la science et la technique résultent de l'activité d'acteurs, de groupes, d'organisations et d'institutions sociales situés, dont l'ambition est de produire des connaissances ou des techniques selon des méthodes éprouvées. On retrouve ici des problématiques qui s'appliquent particulièrement bien à la situation du plateau de Saclay et à la volonté publique de créer un cluster de niveau mondial.

Cette injonction d'innover affecte tout à la fois les secteurs de la recherche et de la formation, mais aussi la société et les pratiques qu'elle développe. L'étude des politiques de promotion de l'innovation et des cadres normatifs mis en place pour son déploiement illustre les ambiguïtés d'un principe qu'on oppose parfois à la précaution. Au-delà des critiques que l'on peut adresser aux théories de l'innovation, leur succès relance un long débat sur le statut de la recherche publique et sur ses retombées économiques. Il incite à renouveler l'interrogation sur le rôle social de la science au sein des réflexions contemporaines sur l'État, l'entreprise, les formes de la collaboration scientifiques et les cadres juridiques et économiques associés.

Enfin, étudier les effets de l'injonction à l'innovation implique une certaine réflexivité et une attention aux transformations des manières de faire et de produire de la science, tout autant qu'aux controverses qu'elle suscite. Un objectif de cet axe consiste donc à analyser les effets de cette injonction sur les systèmes de production des connaissances existants en France, en Europe et plus largement dans le monde, mais aussi sur les systèmes éducatifs, y compris les établissements d'enseignement publics et privés. La MSH Paris-Saclay a ainsi vocation à porter une recherche méthodologique et réflexive interdisciplinaire sur l'innovation, 
les sciences et les techniques, en mobilisant la centaine de spécialistes du domaine au sein du périmètre du plateau de Saclay.

\section{L'activité scientifique}

L'activité scientifique de la MSH Paris-Saclay se décline en deux grands volets :

- La publication d'appels à projets, de cinq types :

- les appels à projets liés à un événement scientifique, appels "Workshops » et « Séminaires »;

- les appels à projets liés à une recherche, appels «Émergence », « Maturation » et « Excellence ».

- La mise en ouvre d'activités scientifiques en propre :

- les Petits Déjeuners Durkheim ;

- les workshops en lien avec les axes de recherche.

\subsection{Les appels à projets : une ingénierie adaptée}

Les appels à projets constituent le cœur des activités de la MSH ParisSaclay, au service des chercheurs et de leurs ambitions. Dès l'été 2016 a été publié un premier appel à projets libre, visant à susciter des objets de recherche interdisciplinaires et interinstitutionnels pour les unités et les chercheurs du périmètre. Il a permis de labéliser neuf projets - colloques, séminaires, recherche - portés par des chercheurs et enseignants-chercheurs appartenant aux trois universités du périmètre (UVSQ, UEVE et UPSud), ainsi qu'à l'ENS Paris-Saclay. $59 \%$ étaient interdisciplinaires avec les sciences de la nature et $24 \%$ concernaient une interinstitutionnalité hors Paris-Saclay.

Des thématiques importantes du périmètre ont ainsi pu émerger, laissant entrevoir les premiers contours des axes actuels. Deux projets se situaient à l'interface des Big Data et des SHS, et un troisième portait sur l'imagerie des matériaux anciens. Les suivants concernaient l'environnement et la santé connectée. 
Forte de cette première expérience, la MSH Paris-Saclay a pu proposer un véritable programme d'appels à projets pour les deux années suivantes.

Ce programme comprend deux catégories d'appels :

- les appels à projets liés à des événements scientifiques, respectivement nommés «Workshops » et «Séminaires ». Le premier labélise une ou deux journées de travail, le second une série de rencontres (minimum quatre) réparties sur plusieurs mois ;

- les appels à projets liés à des recherches, respectivement nommés « Émergence », « Maturation » et « Excellence ». Le premier labélise des recherches naissantes ou en réflexion, le deuxième développe et consolide des recherches déjà structurées, souvent en complément d'autres financements, le troisième vise à labelliser chaque année un projet de grande qualité, reconnu pour son excellence scientifique et pour son interface exemplaire avec les disciplines hors SHS.

Dans un souci de lisibilité et pour permettre aux équipes d'organiser les dépôts de projets dans les meilleures conditions, la MSH Paris-Saclay publie dès l'automne sa programmation annuelle pour l'année suivante. Il s'agit d'offrir à la communauté scientifique une bonne visibilité quant aux possibilités offertes par la structure, chaque appel faisant l'objet de plusieurs dates de retour, par vagues successives.

Les dossiers de candidature sont remplis à partir d'un formulaire type par les porteurs de projets. Une présentation scientifique est exigée, qui explicite les thématiques et objectifs, l'originalité, la méthodologie, les dimensions interinstitutionnelles et interdisciplinaires, la visibilité scientifique de l'équipe, le calendrier de mise en ouvre et le budget prévisionnel du projet.

Les projets proposés font l'objet d'une évaluation soigneuse. Les projets «Workshops ", "Séminaires » et «Émergence » sont évalués à parts égales par les membres du Bureau et par des anciens porteurs de projets. Les membres du Conseil scientifique se concentrent sur l'appel à projets «Maturation ». Les projets « Excellence » sont évalués par des experts choisis en dehors du périmètre Saclaysien. 
Chaque projet est soumis à une double évaluation, non concertée ; une troisième peut être demandée si les deux évaluations reçues sont opposées. Les évaluateurs disposent d'une grille d'analyse définie qui mesure les différents critères retenus (interinstitutionnalité, interdisciplinarité, inscription dans les axes, qualité scientifique, composition et qualité de l'équipe, faisabilité, devenir et envergure du projet, pertinence du budget). L'arbitrage final est réalisé par le Directeur de la MSH Paris-Saclay, après consultation des membres du bureau, excepté pour le projet «Excellence » pour lequel le choix final est effectué par le Conseil scientifique.

Les porteurs reçoivent un courrier du Directeur. Les projets retenus font l'objet d'une publication sur le site internet de la MSH Paris-Saclay, avec un résumé, dans un souci de transparence vis-à-vis de la communauté scientifique. Les projets non retenus sont invités à postuler à nouveau, après avoir pris connaissance de leurs points faibles. L'objectif reste avant tout la labélisation et le financement de projets structurants et respectueux de critères incontournables, notamment l'interinstitutionnalité et l'interdisciplinarité.

\subsection{Les appels liés au soutien d'événements scientifiques}

La MSH Paris-Saclay a également décidé de labéliser des événements scientifiques pour fédérer des recherches et des chercheurs provenant de disciplines et/ou d'institutions différentes, et pour installer la structure dans le " quotidien » des chercheurs SHS du périmètre grâce à une ingénierie adaptée. L'objectif premier est de devenir la structure de référence d'animation de la recherche en SHS du périmètre Paris-Saclay.

Deux appels distincts sont proposés aux chercheurs :

- l'appel à projets « Workshops»;

- l'appel à projets «Séminaires».

Leur labélisation implique un financement et un accompagnement des porteurs de projets en termes de services, qui se manifestent notamment par :

- la création d'outils de communications adaptés : affiches originales, diffusion des événements dans la Newsletter de la MSH Paris-Saclay et sur les réseaux sociaux ; 
- l'accueil des événements : cela concerne l'accueil physique des participants, l'organisation du catering, la mise en place d'un système automatisé d'inscription, etc. ;

- la gestion administrative et financière des fonds attribués, et la réalisation des actes de gestion afférents (missions, commandes etc.) ;

- la publication des actes des événements dans la collection «Actes », dès lors que le porteur fournit les textes ;

- la réalisation de la captation de l'évènement, et la mise en ligne sur la chaîne YouTube de la MSH Paris-Saclay, après montage.

\section{Les appels à projets (AAP) workshops}

L'objectif de l'appel à projets «Workshops » est de permettre à des groupes de chercheurs SHS du périmètre Paris-Saclay de créer leur propre espace de rencontre sur une à deux journées, autour d'un sujet de recherche interdisciplinaire, et de susciter la participation de chercheurs non encore impliqués.

Cet appel connaît un vrai succès, car il répond au besoin de chercheurs, appartenant à des disciplines ou à des institutions différentes, de se concerter autour d'événements scientifiques ponctuels. Utile pour fédérer les recherches en SHS du périmètre de Paris-Saclay, il se révèle également l'outil le plus efficace pour mener une politique de site.

L'appel à projets Workshops a constitué un tremplin pour des projets plus conséquents. Il a permis de faire connaitre la stratégie de la $\mathrm{MSH}$ Paris-Saclay et a contribué à son déploiement sur le site saclaysien. Toutes les universités et écoles du périmètre ont participé à un workshop labélisé MSH Paris-Saclay. Il faut, de plus, noter que la quasi-totalité des workshops ont été interdisciplinaires et/ou interinstitutionnels.

Depuis 2017, la MSH Paris-Saclay a labellisé 50 workshops parmi les 73 déposés.

En termes de répartition des workshops dans les axes de recherche, les résultats sont les suivants :

- 16 s'inscrivent dans l'axe 1 ;

- 15 dans l'axe 2 ; 
- 17 dans l'axe transversal $\mathrm{T}$.

- 2 se situent en dehors des axes de recherche, bien que respectant les critères d'interdisciplinarité et d'interinstitutionnalité.

\section{6 workshops s'inscrivent dans l'axe 1 :}

5 se situent à l'interface des SHS et des problématiques liées au numérique. Ils portent sur la protection de la jeunesse en ligne, les plateformes de micro-travail de tâcheronnat en ligne, la linguistique et les Big Data, ainsi que les questions d'algorithmes et de discrimination.

10 s'intéressent au patrimoine : comparaison des mouvements de jeunesse à la fin des années 1960 dans les Amériques ; le génocide des Tutsis ; les périodiques comme médiateurs dans l'écosystème de la culture visuelle et imprimée ; les traductions de la littérature espagnole en Europe à l'époque classique ; la protection juridique du patrimoine culturel à l'échelle internationale ; le capitalisme, la personnalité et la culture ; l'archéométalurgie expérimentale ; le Design, de la pensée au visible ; Design, l'œuvre de Mel Bochner ; une anatomie de l'Angleterre dans les livres de la première modernité.

Enfin, un workshop a porté sur l'utilisation des données et des méthodologies quantitatives en SHS pour traiter des inégalités en Asie du Sud.

\section{5 workshops ont porté sur les thématiques de l'axe 2 :}

7 portent sur la mobilité et le territoire : enjeux liés à la mobilité électrique ; les usages du numériques et la mobilité urbaine ; la frontière de l'aménagement du territoire, du numérique et de la mobilité ; les stratégies d'investissement des ménages pour leur mobilité ; l'économétrie et les statistiques spatiales; les transports et la mobilité durable.

5 s'intéressent à l'environnement : préservation de la biodiversité ; changements climatiques; design de l'habitat en fonction de contraintes environnementales ; vulnérabilité et résilience dans les approches de développement ; les énergies nouvelles et leur acceptabilité sociale.

3 concernent le thème "santé » : qualité de l'alimentation; dispositifs de $e$-santé ; innovations organisationnelles en Santé mentale». 
Un workshop, a porté sur une approche interdisciplinaire de la structuration de l'espace économique.

\section{7 workshops sont liés à l'axe $T$ :}

3 portent sur les liens entre sciences et société : ce que la demande sociale fait aux sciences sociales ; sciences et émancipation ; un évènement autour des travaux de Susan Silbey (MIT).

7 sur les modes d'innovation : discussion suite à une présentation de Daron Acemoglu (MIT), témoignages d'anciens élèves HEC Paris et le Litem (UEVE) aujourd'hui acteurs de l'innovation ; photographie et design ; obsolescence programmée ; designer dans l'aérospatial ; R\&D and Management.

7 sur l'innovation dans les sciences et à l'université : histoire de l'évolution des sciences sociales (saisons 1 et 2) ; obsolescence programmée ; conséquences des innovations en matière de gestion des universités autour d'une présentation de Sheila Slaughter (Université du Wisconsin ; saison 1 et 2) ; conférence de sociologie filmique ; les apports de Robert Salais à la recherche.

Enfin la MSH Paris-Saclay a labellisé deux workshops interdisciplinaires qui n'entraient pas dans ses axes de recherche stricto sensu, sur la médiatisation de l'islam et des musulmans et les déviances et violences sexuelles.

\section{Les appels à projets «Séminaires »}

L'objectif de l'appel à projets Séminaires est de soutenir des groupes de chercheurs dont les participants proviennent de différentes unités $\mathrm{du}$ site et se réunissent régulièrement autour de thématiques SHS interdisciplinaires. Ces cycles de rencontres ont une vocation plus pérenne que les workshops. Des séminaires existant depuis plusieurs années ont pu être labélisés et, ainsi, se développer en termes de visibilité. Cet appel à projets, apprécié par la communauté, a permis l'ancrage de la structure dans des groupes de travail aux réunions régulières.

L'axe 1 est représenté par cinq séminaires qui concernent les humanités : "Patrimoine et patrimonialisation » (saisons 1 et 2); «Médias et médiations de la gastronomie » (saisons 1, 2 et 3) ; «Identités 
culturelles nationales et transnationales hispano-caribéennes et hispanocontinentales » (saisons 1, 2, et 3) ; «Les libertés numériques », «Les périodiques comme médiateurs culturels ».

L'axe 2 est représenté par deux séminaires qui concernent l'environnement et le territoire : "Prévision, précaution, préventions et catastrophe » et «Conflits et territoires » (saisons 1 et 2 ).

L'axe $\mathrm{T}$ est représenté par trois séminaires qui mènent une réflexion sur l'innovation : «Histoire du design », «Design, écologie et nouvelles normativités »; «Recherche et innovations responsables : éclairages interdisciplinaires », et par trois séminaires sur les relations entre science et société : «Savoir et pouvoir »; "Relations science-société (saison 1, 2 et 3 )» et «Academic all star game » qui traite de l'innovation en sciences de gestion.

Un dernier séminaire étudiant les questions politiques liées au genre dans le monde carcéral a fait l'objet d'une labélisation lors des appels 2017, 2018 et 2019 et bientôt d'une publication dans la collection actes de la MSH en 2020.

\subsection{Les appels liés au soutien de projets de recherche}

La deuxième catégorie d'appels à projets proposée par la MSH ParisSaclay concerne le soutien de projets de recherche. Il s'agit d'accompagner le développement de recherches déjà existantes, mais aussi d'en susciter de nouvelles.

L'objectif de ces appels est de servir d'appui aux chercheurs afin qu'ils s'insèrent ou créent des réseaux de recherche d'envergure internationale de premier plan. La MSH Paris-Saclay souhaite ainsi voir émerger des recherches interdisciplinaires, notamment avec les sciences techniques et les sciences de la vie et de l'ingénieur. Cette ambition doit permettre la réalisation de publications de rang élevé, mais aussi susciter des réponses à des appels à projets plus importants.

Ainsi, les financements des projets de recherche sont conçus comme des tremplins vers des appels nationaux et internationaux, notamment européens. Les porteurs des projets Émergence sont invités à candidater aux appels à projets Maturation, alors que les porteurs de projets 
«Maturation » sont suivis individuellement et incités à aller vers des financements plus notables (ANR, Europe, etc.).

Pour les accompagner, la MSH Paris-Saclay les informe des différentes possibilités de financements et les incite à participer aux séances de formation et/ou d'informations proposées par les différents partenaires (CNRS, UPSaclay, ANR, Mesri, etc.). Elle s'appuie notamment sur le Groupe de travail (GT) ValoSHS/Europe initié par l'UPSaclay auquel elle participe, et qui regroupe les chargés de valorisation « Europe » des établissements et instituts du périmètre.

\section{Les appels à projets «Émergence »}

Cet appel à projets est destiné à favoriser le développement de nouvelles recherches construites à partir de coopérations interdisciplinaires et interinstitutionnelles. L'objectif est d'impulser des recherches qui n'impliquent pas nécessairement l'organisation d'un événement scientifique. Depuis 2017, nous avons labélisé 17 projets de ce type.

L'axe 1 affiche neuf projets «Émergence », à l'interface du numérique et des humanités, qui portent sur l'exploration de changements sonores grâce à l'intelligence artificielle ; l'intelligence des algorithmes et les stéréotypes ; les musiques de sortie de guerre ; le génocide des Tutsis ; la circulation photographique du $\mathrm{XIX}^{\mathrm{e}}$ au $\mathrm{XX}^{\mathrm{e}}$ siècle ; la justice prédictive et les algorithmes ; le bitcoin du peuple, le cas argentin ; les algorithmes, plateforme et concurrence ; et la Préparation à un projet ERC sur Big Data et attitudes face au risque.

L'axe 2 a vu éclore deux projets portant sur les territoires d'interface et la biodiversité urbaine en Île-de-France, et le programme de recherche interdisciplinaire sur les mobilités urbaines et péri-urbaines.

Quatre projets «Émergence » entrent dans l'axe $\mathrm{T}$ : ce que la demande sociale fait aux sciences sociales ; étude des fablabs et tiers lieux d'innovations ; communautés et pratiques communautaires, les fablabs dans la perspective de la transition écologique ; le travail des innovateurs à l'épreuve de l'éthique.

Enfin, deux projets ont permis la création d'observatoires du droit à partir de recherches sur les actions de groupe et l'éthique en entreprise. 
Les appels à projets « Maturation »

Les appels à projets Maturation ont pour but d'approfondir des recherches déjà existantes au sein de l'UPSaclay et de favoriser leur développement. Il s'agit de favoriser la production de livrables de différentes natures : publications, grands événements scientifiques, partenariats internationaux, etc.

Ces projets plus importants (financement de $25 \mathrm{k} €$ maximum par projet) sont évalués par les membres du Conseil scientifique.

L'axe 1 est représenté par huit projets :

- "Reconstruction de la distribution de l'ensemble des choix à partir de données de choix discret : une approche de type "Compressed Sensing" »;

- "Analyse des comportements interactionnels liés à l'engagement de l'utilisateur pour le design de robots socialement compétents »;

- «ELEMENTA », sur l'étude des conséquences cognitives sur des individus confrontés à une expérience suscitant l'anthropomorphisme avec des éléments naturels ;

- «Exploiting the Informational Content of Large Data sets in Economics: Causal Inference and nowcasting ";

- «DipLab », qui étudie le profil et les stratégies des microtravailleurs chez le leader français du microtravail sur plateforme numérique ;

- «Les algorithmes intelligents sont-ils racistes et sexistes et comment leur apprendre à ne pas l'être ? »;

- «Transatlantic cultures », qui propose de mettre en œuvre une histoire culturelle connectée de l'espace atlantique, grâce à des supports et outils numériques ;

- «HistorIA », dont l'objectif est d'approfondir l'étude des langues au moyen de l'IA sur des données massives d'usage de la langue. 
Trois projets correspondent à l'axe 2 :

- «Impact de l'évolution environnementale dans les politiques d'aménagement du territoire : le cas de la biodiversité »;

- «Psychofood », qui étudie le lien entre régime alimentaire et profil psychologique des consommateurs ;

- «JUICCE », qui porte sur le lien entre justice sociale et vulnérabilité climatique.

Les appels à projets «Excellence»

L'appel à projets Excellence a pour objectif de promouvoir des projets à fort potentiel, portés par des chercheurs issus d'institutions et de disciplines différentes de la communauté Paris-Saclay.

Il est destiné à encourager une coopération scientifique d'excellence à fort enjeu sociétal entre chercheurs SHS et au-delà. Il peut s'agir d'équipes qui ont déjà travaillé ensemble et souhaitent approfondir leur projet de recherche commun, en vue notamment de répondre à des appels à projets plus importants.

Les projets doivent s'ouvrir à la collaboration avec les disciplines hors Sciences Humaines et Sociales, même si les SHS restent dominantes. Ils doivent également présenter une interface avec les problématiques $\mathrm{du}$ territoire saclaysien, et comporter des déclinaisons possibles pour les acteurs du site (entreprises, personnels, associations, collectivités locales, usagers, etc.).

En 2019, 11 projets ont été présentés à l'appel à projets Excellence. Le Conseil scientifique a finalement retenu le projet «TerriBio Saclay » (Territoires d'interface et biodiversité urbaine sur le plateau de Saclay), qui est centré sur l'analyse de la biodiversité urbaine et cultivée comme vecteur de représentations sociales et comme objet pour l'action publique locale. Les recherches sont menées sur le plateau de Saclay, territoire marqué par une forte dynamique d'artificialisation et des enjeux importants en termes de coexistence et d'utilisation des espaces urbains et des ouverts, agricoles ou semi-naturels, et de la biodiversité. Le projet associe une équipe pluridisciplinaire en SHS (sociologie, géographie) avec un collectif de chercheurs travaillant en écologie et à l'interface entre les SHS et les 
sciences de la vie (ethnoécologie, psychologie de l'environnement).

Il est envisagé d'installer les futures équipes titulaires de projets Excellence dans les locaux de la pépinière de talents de la MSH, installée dans les bâtiments de l'ENS Paris-Saclay, à partir de 2020. Les équipes intéressées pourront y trouver un soutien matériel, une aide au quotidien pour leurs problèmes administratifs ou logistiques, un accompagnement en matière de formation, ainsi qu'une expertise en matière de contacts ou de liens aux grands programmes nationaux et européens. L'éventualité d'association et d'invitation de chercheurs étrangers à ces projets, au sein de chaires d'excellence de l'ENS Paris-Saclay ou autres, sera explorée.

\subsection{Des activités propres}

Parallèlement à ses appels à projets, la MSH Paris-Saclay a mis en place des activités scientifiques propres, dont elle a totalement assuré le montage, l'organisation et la valorisation.

\section{Les Petits Déjeuners Durkheim}

Dès 2016, la MSH Paris-Saclay a pu reprendre à son compte les petits déjeuners Durkheim, mis en place depuis quelques années par l'ENS Paris-Saclay.

Le concept est simple : autour d'un petit déjeuner, un vendredi matin par mois, de janvier à juin, chercheurs, enseignants, étudiants et tout public intéressé sont invités à débattre, suite à la présentation d'ouvrages issus des travaux de recherche menés dans les laboratoires SHS affiliés à la MSH Paris-Saclay. Depuis 2019, les Petits déjeuners sont inscrits au programme de l'École doctorale SHS de l'université Paris-Saclay.

Ainsi, depuis 2016, 24 séances ont été programmées (6 par saison). Depuis 2017, elles font l'objet de captations, accessibles à tous sur la chaîne YouTube de la MSH Paris-Saclay.

\section{Les workshops liés aux axes de recherche de la MSH Paris-Saclay}

L'année 2017 a été l'occasion de finaliser le descriptif des axes de recherche. Cette finalisation s'est réalisée en deux temps, et s'est fondée sur la communauté SHS du périmètre. 
Dans un premier temps, lors du colloque inaugural du 27 janvier 2017, des ateliers thématiques ont permis aux participants de discuter librement des cinq axes alors pressentis. Dans un deuxième temps, quatre workshops ont été organisés autour des projets, entre mars et juin 2017. Ouverts aux chercheurs et enseignants-chercheurs du périmètre, ces workshops ont été l'occasion pour les participants de discuter des travaux en cours au sein de leurs unités. Selon les cas, ils ont rassemblé entre 30 personnes et 45 personnes. Ils ont fait l'objet de captations, que l'on peut retrouver sur la chaîne YouTube de la MSH Paris-Saclay.

C'est à l'issue de ces deux séries de rencontres que le Directeur Stefano Bosi, secondé par les membres du bureau, a pu proposer un projet modifié autour des trois axes décrits précédemment.

\section{La structure de gouvernance}

L'activité intense et soutenue de la MSH Paris-Saclay repose sur une structure de gouvernance particulière, qui associe les instances de pilotage externes et la mise en œuvre de pôles d'activités en interne. En effet, en tant qu'unité de service, elle assure différents types de services aux porteurs de projets, qui se répartissent au sein de quatre pôles, encore en cours d'élaboration : administratif et financier, communication, édition et accompagnement scientifique.

\subsection{Les quatre piliers de la gouvernance}

Le fonctionnement de la MSH Paris-Saclay repose sur quatre piliers, qui assurent sa bonne gouvernance :

- le Conseil scientifique : garant de l'orientation scientifique de la MSH Paris-Saclay, il constitue une force de proposition de ses évolutions, en contribuant à définir les grandes orientations scientifiques et à identifier des relais scientifiques internationaux ;

- le Comité de pilotage : à ce jour composé de quatre tutelles, il doit se confronter à des défis tels que l'entrée éventuelle d'autres tutelles ou le partenariat de la MSH Paris-Saclay avec les établissements de Polytechnique, etc. ; 
- le Bureau : en fonction de leurs disciplines et expériences, les membres du Bureau participent aux orientations, conseillent le Directeur et apportent leur capacité d'expertise sur les projets proposés. Leurs spécialités correspondent également aux axes de la MSH ;

- l'Assemblée générale des personnels : la réussite du projet s'appuie sur la cohésion et l'investissement personnel et collectif des collaborateurs, qui participent à son essor par leur opérationnalité et leur connaissance du terrain.

\subsection{Pôle administratif et financier}

La MSH Paris-Saclay a choisi de garder la maitrise d'ouvrage totale des crédits attribués. Cela signifie que l'ensemble des actes de gestion liés aux projets est réalisé en interne et qu'il n'y a aucun transfert de fond dans les unités des porteurs.

Ce choix répond à un double objectif :

- s'assurer que les dépenses d'un projet correspondent globalement au budget qui avait été présenté et évalué lors de l’appel ;

- contribuer à « autoalimenter » les ressources du laboratoire, considérant qu'en moyenne $30 \%$ des crédits affectés à un projet ne sont pas dépensés.

Afin de gagner en efficacité, la MSH Paris-Saclay transfère l'ensemble de ses crédits au CNRS, de sorte que l'assistante administrative et financière de l'unité n'ait pas besoin de maitriser l'ensemble des outils de gestion des quatre tutelles. Il est possible de créer une "entité dépensière » pour chaque projet, et donc :

- d'assurer un suivi efficace des crédits attribués, sans risque de dépassement ;

- d'indiquer aux porteurs de projets en temps réel leur solde disponible, avec le détail de l'ensemble des dépenses engagées. 


\subsection{Pôle Communication}

\section{Les enjeux en termes de communication}

Jeune structure créée au 1er janvier 2015, la MSH Paris-Saclay a mis en œuvre une stratégie de communication pour développer sa présence dans le paysage saclaysien, afin de :

- se faire connaître dans un périmètre comprenant beaucoup d'autres structures de recherche, et des sites distants (Versailles, Gif-sur-Yvette, Évry, Cachan, Orsay, Sceaux, Meudon, etc.) ;

- développer la visibilité des SHS sur un territoire où les effectifs dans les autres sciences sont les plus importants;

- faire connaitre ses actions et dispositifs de financement à tous les chercheurs SHS (une visite des 37 laboratoires concernés avec présentation de la MSH, appelée le «MSH tour »;

- développer la visibilité des événements qu'elle soutient (augmenter la fréquentation des événements, les faire connaitre, valoriser les résultats);

- rendre compte de son action à ses tutelles et partenaires, ainsi qu'au Réseau National des MSH.

La MSH Paris-Saclay utilise notamment les outils de communication suivants :

- un site web institutionnel avec une fréquentation quotidienne moyenne de 200 vues (111 000 vues depuis son lancement en janvier 2017) ;

- des réseaux sociaux comme Facebook, Twitter, LinkedIn et Instagram ;

- une newsletter mensuelle distribuée à plus de 2000 inscrits (enseignants, chercheurs, institutionnels, doctorants, etc.) du périmètre Paris-Saclay ;

- une chaîne YouTube avec les vidéos des manifestations scientifiques captées ;

- des supports de communication divers : pour la MSH 
proprement dit (plaquette de présentation, projet scientifique) et pour les événements labellisés (production d'affiches et goodies, aide à la mise en place de sites web événementiels sur SciencesConf, etc.).

\section{Soutien aux laboratoires}

Pour chacun des événements qu'elle labellise, la MSH Paris-Saclay propose aux organisateurs un ensemble de services de logistique, d'assistance à la création de sites événementiels, de mise en place d'un système d'inscription automatisé, de réalisation d'affiches et d'autres supports de communication (programmes, flyers, etc.) par un graphiste professionnel. Cette politique de communication a permis de donner aux manifestations soutenues une identité visuelle forte et désormais reconnue sur le périmètre Paris-Saclay. Elle permet également à la MSH Paris-Saclay et aux organisateurs de disposer de supports de communications de qualité pour communiquer sur leurs sites et réseaux sociaux. Ces services sont également proposés aux deux revues hébergées par la MSH Paris-Saclay.

\section{Annuaire des chercheurs SHS}

La MSH Paris-Saclay a mis en place un annuaire en ligne des chercheurs SHS du périmètre Paris-Saclay. L'objectif est d'optimiser la cartographie des chercheurs par discipline, objets et techniques de recherche et de retranscrire ces éléments dans un annuaire interactif en accès libre sur le site de la MSH.

Il est ainsi possible de consulter les profils de la plupart des 1200 chercheurs répartis dans 37 laboratoires et d'effectuer des recherches par mots clés sur leurs disciplines et objets de recherche. Cet annuaire permettra aux chercheurs de trouver des ressources et expertises pour initier ou développer des projets interdisciplinaires, ou d'être sollicités sur des propositions de collaboration. Une campagne d'inscription à l'annuaire a été lancée à l'automne 2018. Plus de 500 chercheurs figurent à ce jour dans l'annuaire. 


\subsection{Pôle éditorial}

Un des objectifs de la MSH Paris-Saclay est la constitution d'un pôle éditorial dédié au soutien de revues scientifiques en SHS et à la valorisation par la publication des travaux issus de recherches labélisées par l'unité, en accord avec les recommandations du réseau national des $\mathrm{MSH}$ et de l'InSHS.

À ce jour, ce pôle s'organise autour de trois activités :

- l'hébergement de revues scientifiques;

- la publication d'actes de manifestations scientifiques;

- l'édition de monographies.

\section{L'hébergement de revues scientifiques}

Cet hébergement se réalise dans le cadre de la politique du CNRS, qui favorise l'installation des revues et des éditeurs associés dans les MSH.

L'hébergement de revues apporte les services suivants :

- la collecte des contenus et leur vérification ;

- la mise aux normes éditoriales et les vérifications typographiques, orthographiques, syntaxiques et grammaticales des textes ;

- le stylage et la mise en page du numéro ;

- l'élaboration de la couverture ;

- la coordination de l'objet éditorial (print et web), avec production du fichier html ;

- la communication.

La MSH Paris-Saclay héberge aujourd'hui deux revues.

\section{terrains \& travaux}

Créée en 2000, terrains \& travaux est une revue semestrielle de sciences sociales. D'aspiration généraliste, elle publie des articles originaux, principalement en sociologie et science politique, mais aussi en histoire, anthropologie et économie. Les textes, ancrés dans des enquêtes empiriques, donnent à voir les matériaux collectés et les méthodes mobilisées, tout en s'appuyant sur une solide réflexion théorique. 
Chaque numéro comprend un dossier thématique d'une dizaine d'articles, pour lequel un appel à contributions est largement diffusé, ainsi qu'un ou deux articles hors dossier. La revue publie également des notes critiques et des traductions inédites de textes importants, en rapport avec le thème du dossier.

Les articles reçus sont évalués en double aveugle par deux lecteurs (membres du Comité de rédaction ou externes), puis discutés collectivement par l'ensemble du Comité. Chaque soumission fait systématiquement l'objet d'un rapport synthétique à l'intention de l'auteur, qui reçoit une réponse dans un délai de quatre mois maximum.

terrains \& travaux est une revue classée dans la liste des revues SHS du HCÉRES, en sociologie et science politique. La revue bénéficie du soutien de l'InSHS du CNRS.

Cinq numéros ont été publiés depuis l'implication de la MSH ParisSaclay :

- Varia (n 30, 2017/1)

- Consommer autrement? (n० 31, 2017/2)

- Les Gouvernements du corps (nº 32, 2018/1)

- Empreintes et emprises de la financiarisation ( $\left.{ }^{\circ} 33,2018 / 2\right)$

- Socialisation professionnelle et circulations dispositionnelles ( $\mathrm{n}^{\circ} 34$, 2019/1)

\section{L'Homme \& la Société}

La revue L'Homme \& la Société, pluridisciplinaire dès sa création en 1966, s'adresse à toutes les disciplines des sciences sociales - son comité est composé de sociologues, d'historiens, d'économistes, d'anthropologues, de juristes, mais aussi de philosophes et de spécialistes de la littérature et affiche une ambition de critique des processus contemporains de naturalisation des acteurs et des sociétés et de déchiffrement des initiatives et alternatives productrices d'émancipation et d'autoémancipation individuelle et collective.

L’Homme \& la Société vise à élucider les déterminations de l'activité sociale, ses oppressions, mais aussi les ouvertures qui s'y révèlent pour les acteurs. La revue s'attache ainsi à décrire et analyser les articulations entre 
《liberté » et « déterminations », dans la perspective d'un matérialisme non déterministe. Elle publie des numéros thématiques, ainsi que des articles hors dossiers variés, un éditorial, une revue des revues et des recensions d'ouvrages reçus.

Cinq numéros ont été publiés depuis l'implication de la MSH ParisSaclay :

- Corps sexué, corps genré : une géopolitique (n 203-204, 2017/1-2)

- L'Homme-Machine I. Le travailleur-machine (n' 205, 2017/3)

- À quoi servent les droits aujourd'bui ? (n 206, 2018/1)

- L'Homme-machine II. Du travailleur augmenté à l'bomme augmenté (n' 207, 2018/2)

- Sexualités minoritaires. Expériences subjectives, communautés érotiques et politiques de reconnaissance ( $\mathrm{n}^{\circ} 208,2018 / 3$ )

Les Actes de colloques

La MSH Paris-Saclay a lancé en mars 2018 la collection «ACTES », destinée à publier les actes des événements scientifiques (workshop, colloques, séminaires, etc.) labélisés dans le cadre de ses appels à projets. Pour ce faire, elle est devenue maison d'édition, sous le nom MSH ParisSaclay Éditions.

Les responsables des workshops jugés les plus intéressants ou qui ont attiré le plus large public sont contactés, avec une proposition de publication rapide de leurs actes. Si le projet est retenu, il est laissé quelques mois aux auteurs potentiels pour réaliser une version écrite de leur communication, relue et vérifiée sous la responsabilité des éditeurs du volume avant d'être remise. La MSH Paris-Saclay se charge ensuite des opérations liées à la publication des actes (suivi de la chaîne éditoriale et de la fabrication).

Les ouvrages sont disponibles en accès libre en version numérique sur le site de la MSH Paris-Saclay. Des exemplaires papiers ont également vocation à être diffusés largement, gratuitement.

Le premier volume, paru en mars 2018, rassemble les communications réalisées lors de la journée d'étude «Sciences humaines et sociales \& 
Photovoltaïque », organisée par le Club de l'Orme le 25 avril 2017, avec le concours du CEA, du département SHS de l'Université Paris-Saclay, de l'Alliance ANCRE et de l'Institut de recherche en photovoltaïque du plateau (IPVF).

Un deuxième numéro, consacré au séminaire «PREDICT - Biologie prédictive pour la santé » est paru en octobre 2018.

Publié en décembre 2018, le troisième volume rassemble les articles d'auteurs présents à l'atelier scientifique SHS-Mobilités du CEA Saclay, sur le thème des « Nouvelles mobilités à la lumière des sciences humaines et sociales ».

Sociologues et économistes face à la demande de savoirs. Participation et contournements, le quatrième numéro des actes, est sorti en octobre 2019. Il concentre les articles d'auteurs qui ont participé à une journée d'études et un colloque international, organisés à l'UVSQ.

\section{Les Éditions EMSHA}

Les MSH Paris Nord, Paris-Saclay et Nanterre ont mis en place un partenariat éditorial et créé les «Éditions des MSH associées. Nanterre, Saclay, Saint Denis » (EMSHA). Elles ont été lancées le 12 avril 2018 dans le cadre d'un événement commun.

Cette nouvelle maison d'édition se donne comme mission d'offrir aux chercheurs des trois MSH la possibilité de publier des ouvrages en SHS, sous la forme de livres électroniques (e-books) disponibles à travers l'espace éditorial EMSHA ouvert sur la plateforme de publication « OpenEdition Books » développée par le Centre pour l'édition électronique ouverte (Cléo).

La direction éditoriale est assurée par un comité éditorial composé de six personnes - deux représentants par MSH dont directeur/directrice et directeur/directrice adjoint(e) - chargé d'élaborer la politique éditoriale.

L'objectif de cette collection est, avant tout, de favoriser l'interdisciplinarité. Les services éditoriaux offerts par les Éditions EMSHA sont les suivants :

- conseils et expertise pour les auteurs (présentation des collections EMSHA ainsi que des contrats liés aux droits 
d'auteur, présentation des conditions techniques et scientifiques d'acceptation des ouvrages, fourniture de recommandations orthotypographiques) ;

- corrections, vérification et mise aux normes des ouvrages, vérification des éléments (bibliographies, images, etc.);

- stylage selon une feuille de style prédéfinie ;

- soumission des ouvrages au conseil scientifique de l'EMSHA pour validation ;

- mise en ligne et publication des ouvrages sur Open Edition Books.

\subsection{Pôle Coordination scientifique}

L'accompagnement scientifique des chercheurs et enseignants-chercheurs du périmètre se concrétise de différentes façons, de l'amont jusqu'à l'aval du développement de leur projet de recherche : rédaction des appels de la structure, recrutement des experts, arbitrage, contact des chercheurs.

En amont, il consiste à conseiller et accompagner les éventuels porteurs de projets dans la préparation de leurs dossiers. Le responsable de la coordination scientifique facilite les interactions entre les scientifiques, notamment quand il s'agit de renforcer les aspects «interdisciplinarité » et « interinstitutionnalité » des projets proposés. En aval, il consiste à s'assurer que le projet se déroule correctement, d'identifier les retards éventuels et de proposer le cas échéant des solutions adaptées.

De façon générale, il s'agit de d'accompagner le développement de l'activité scientifique par :

- la diffusion des appels à projets nationaux et internationaux accessibles, en assurant une veille en adéquation avec les axes de recherche de la MSH Paris-Saclay;

- le repérage des projets labélisés suffisamment matures pour envisager un développement plus ambitieux en répondant à des appels de type ANR ou H2020 ;

- l'aide au montage administratif et financier de ces projets, dans une logique d'ingénierie de projet, en lien avec la délégation régionale du CNRS ; 
- la participation à différents groupes de travail locaux ou nationaux ;

- l'interface avec les acteurs de la valorisation de la recherche (CVT Athena, SATT Paris-Saclay, Direction de l'Innovation de l'Université Paris-Saclay) ;

- la coordination du déploiement d'outils nationaux développés par le CNRS au sein du périmètre Paris-Saclay, (PROGEDO, avec la mise en place d'une PUD), Huma-Num, avec la diffusion des différents dispositifs proposés par cette TGIR, etc.).

\section{Et maintenant ?}

En quelques années la MSH Paris-Saclay s'est imposée comme un acteur de référence du monde de la recherche, capable à la fois d'impulser des travaux et des collaborations entre équipes et disciplines des SHS, ainsi que d'assurer leur reconnaissance et de faciliter les passerelles avec les autres types de recherches présentes au sein de l'Université Paris-Saclay et plus largement sur le périmètre saclaysien.

Force est de constater que la MSH a su se positionner en tant que fédérateur de la communauté SHS de son territoire. Les réponses fructueuses aux appels à projets, combinées à ses activités scientifiques propres, la positionnent comme le fer de lance de la structuration des SHS, souvent en lien avec les sciences dures. De nombreuses coopérations scientifiques ont ainsi pu émerger sous son égide, alors que de nouveaux objets de recherche sont apparus ou ont trouvé leur reconnaissance dans le cadre des trois grands axes de recherche définis à partir des thématiques porteuses du périmètre saclaysien.

L'hébergement de la MSH dans les nouveaux locaux de l'ENS Paris-Saclay, à partir de 2020, doit être conçu comme une opportunité. Il permettra aux chercheurs et aux personnels d'accompagnement de la recherche de bénéficier d'un environnement de qualité, ainsi que d'infrastructures modernes et fonctionnelles (salles de réunion, amphithêâtres, espaces de co-working, etc.). Associés aux objectifs 
scientifiques et aux dispositifs de recherche encore à développer, les moyens disponibles contribueront à donner vie à ce projet et à cette ambition pour les SHS, en termes de gouvernance, de ressources humaines et financières, ainsi que d'infrastructure logistique.

Le prochain quinquennat permettra ainsi de conduire à un développement de ces activités et à une extension des outils au service de la recherche, afin de permettre à la MSH Paris-Saclay de maintenir un rythme de croisière élevé, adapté aux attendus de la communauté scientifique du périmètre saclaysien et à ses ambitions de rayonnement. Les entretiens qui suivent, réalisés par Sylvain Allemand, rédacteur en chef de Paris-Saclay Le Média, révèlent à la fois le travail accompli et ouvrent des perspectives fructueuses pour l'avenir. 
ENTRETIENS AVEC

LES TROIS DIRECTEURS

DE LA MSH PARIS-SACLAY 


Directeur de recherche CNRS, sociologue, spécialiste de droit du travail, il a été en charge du chantier de préfiguration de la MSH Paris-Saclay qu'il a ensuite dirigée, de sa création jusqu'en août 2016.

II est, depuis, chercheur au Centre Maurice Halbwachs (CNRS, ENS Ulm, EHESS), après avoir été membre du laboratoire IDHES (antenne de Cachan). II n'en continue pas moins à cultiver des liens avec la MSH : les 19-20 septembre 2019 , il organisait, avec son soutien, un workshop en l'honneur de l'économiste Robert Salais.

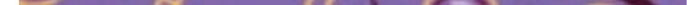




\section{Claude DIDRY \\ Directeur de recherche CNRS, au CMH (UMR 8097) \\ Ex-directeur de la MSH Paris-Saclay}

- Pouvez-vous, pour commencer, expliquer ce qui vous a prédisposé à la création d'une MSH?

Je mettrai naturellement en avant le caractère interdisciplinaire de ma formation, que j'ai poursuivie notamment au sein de l'EHESS au contact de mes codirecteurs de thèse : Antoine Lyon-Caen (mon directeur officiel), professeur de droit à Nanterre et directeur d'études à l'EHESS, et Robert Salais, statisticien économiste, directeur du Groupement de recherche (GdR) IEPE (Institutions, Emploi et Politiques économiques), ainsi que d'éminents enseignants-chercheurs comme l'historien Gérard Noiriel dont j'avais suivi les enseignements en DEA à l'ENS, ou encore Luc Boltanski, qui avait encadré mon mémoire. J'ai baigné dans un environnement d'autant plus stimulant qu'il fut irrigué par une vraie dynamique d'échange entre l'économie, la sociologie, l'histoire, le droit, etc.

- Nous reviendrons à Robert Salais, auquel vous avez récemment consacré un colloque soutenu par la MSH Paris-Saclay. Mais ne brûlons pas les étapes. Revenons aux circonstances qui vous ont amené à participer à la création de celle-ci...

La proposition qui me fut faite de diriger cette nouvelle institution intervint au terme d'un processus qu'il faut replacer, pour le comprendre, dans le contexte général du projet de création de l'université Paris-Saclay. Dans sa version initiale, ce projet privilégiait les sciences exactes et les sciences de 
l'ingénieur sur les Sciences de l'Homme et de la Société (SHS). Il était de toute évidence conçu par des scientifiques pour des scientifiques, qui, de surcroît, se connaissaient déjà, avaient l'habitude de travailler ensemble. Un rapprochement avec les SHS n'était pas un impératif, à leurs yeux, c'est le moins qu'on puisse dire. Reconnaissons que nous-mêmes, chercheurs en SHS, avions peut-être un tropisme parisien, qui ne nous inclinait pas à saisir l'intérêt ni la nécessité de quitter Paris ou la toute proche banlieue pour le Plateau de Saclay... C'est dans ce contexte fait d'incompréhension réciproque entre des sciences dites "dures", peu intéressées par les SHS, et des SHS, elles-mêmes peu sensibles à la dimension technologique de la recherche, qu'un premier projet de MSH, porté par Pierre-Paul Zalio et Éric Godelier, vit le jour, mais sans aboutir.

- À quel moment intervenez-vous dans le processus?

Dès 2011-2012, dans le cadre d'une mission que m'avait confiée le président de l'ENS Cachan, Pierre-Paul Zalio, quand le projet de création d'une Comue s'est précisé [elle sera officiellement créée en 2014]. Je me suis occupé alors simultanément du département SHS ayant vocation à prendre en charge le volet formation de la future université Paris-Saclay, et de la création de la MSH, dédiée, elle, à la recherche.

\section{" Par comparaison avec l'univers des sciences dites "dures", rompues à la coopération scientifique, celui des SHS était éclaté, dans une situation que le sociologue que je suis n'hésiterait pas à qualifier d'“anomique" 》}

- Aviez-vous déjà une idée de ce que les SHS représentaient dans le cadre de la Comue?

Non, autant le dire. À l'époque, il n'y avait pas encore eu de recensement systématique des unités de recherche existantes. Par comparaison avec l'univers des sciences dites "dures", rompues à la coopération scientifique, celui des SHS était éclaté, dans une situation que le sociologue que je suis n'hésiterait pas à qualifier d'« anomique ». L'urgence était donc de 
faire en sorte que les chercheurs de ces disciplines se rencontrent et d'inventorier les forces sur lesquelles nous pourrions nous appuyer.

Durant l'année 2012, nous consacrâmes beaucoup de temps à réunir des directeurs d'unités de recherche pour essayer, sous l'impulsion de Jacques Commaille, de dégager les lignes de force de la recherche en SHS à Paris-Saclay.

C'est ainsi que nous eûmes l'agréable surprise de découvrir que ces dernières réunissaient près de 1000 chercheurs et enseignantschercheurs, et encore, sans compter les doctorants. Sur un effectif total de 10000 enseignants-chercheurs pour l'ensemble de la Comue, cela était tout sauf négligeable.

C'est à partir de cette même année 2012 qu'émergèrent des individualités appelées à m'accompagner dans la création et l'animation de la future MSH : Laurent Willemez, professeur de sociologie à I'UVSQ, à qui je confiai la tâche de gérer le département SHS ; Stefano Bosi, économiste, directeur adjoint scientifique de l'InSHS ; enfin, André Torre, également économiste, directeur de recherche à l'Inra. Ensemble, nous avons commencé à dresser un panorama des thématiques à privilégier, en partant, j’insiste sur ce point, sur l'existant.

- Le faisiez-vous en prenant en compte la particularité de l'écosystème technologique dans lequel la MSH était appelée à s'inscrire?

Oui, bien sûr. Cela fut même au cœur de nos réflexions. Moi-même, au travers de mes recherches en sociologie, j'en étais venu à m'intéresser aux modalités du travail scientifique, de recherche et d'innovation, et ce, au sein de structures publiques aussi bien que privées. C'est dire si le contexte territorial de Paris-Saclay, constitué en écosystème, ne m'était pas indifférent !

- Aviez-vous le sentiment de partir d'une page blanche en considérant que la MSH Paris-Saclay devait être spécifique du fait du contexte qu'on vient d'évoquer?

Jacques Commaille, alors président du conseil d'orientation du Réseau national des Maisons des Sciences de l'Homme [2001-2005], m'avait 
donné un exemplaire de l'ouvrage qu'il avait dirigé en 2006, sous le titre Avenir de la recherche et Maisons des Sciences de l'Homme. Sa doctrine dite des « $5 \mathrm{i}$ » fut mon « kit de montage » de la MSH Paris-Saclay...

- Des « $5 i » ?$

Oui, un premier « $\mathrm{i}$ » pour, comme il se doit, "Interdisciplinaire », les suivants pour «Inter-établissement » (les projets soutenus par une MSH doivent impliquer des équipes de plusieurs établissements), «Inscription territoriale » (en l'occurrence à un écosystème, ainsi que nous le disions), Internationalisation, enfin... Ah, mince ! je crains, d'avoir oublié le cinquième $« \mathrm{i} » \ldots$

- Innovation ? Incubation ?...

Non...

- Incitation?

Cela aurait pu être cela, mais voilà, cela me revient, il s'agit d'«Identité spécifique »! Si des MSH partagent des fonctions communes, elles sont aussi invitées à cultiver des spécificités en assumant des fonctions nationales dans le cadre d'une division du travail au sein du réseau.

- Ence quiconcerne la vôtre, ils'agissait donc defavoriser une interdisciplinarité "forte » entre SHS et recherche scientifique et technologique?

Disons « assez forte ». Cette ouverture à la recherche scientifique et technologique me renvoie à un entretien que j'eus en 2007 avec JeanYves Mérindol, le prédécesseur de Pierre-Paul Zalio, à la tête de l'ENS Cachan. Il avait fait un parallèle avec le MIT, en me proposant de créer le volet sciences sociales du MIT à la française que représentait pour lui Paris-Saclay. Il avait une analyse très approfondie de la situation, mais j'avais décliné l'offre, considérant qu'elle était alors incompatible avec mon engagement dans la recherche. 
- Étant entendu qu'il ne s'agissait pas pour les SHS de venir en appui des sciences dites "dures", mais de pouvoir prendre l'initiative de projets interdisciplinaires à travers des dispositifs en forme d'appels à projets...

Ce fut très exactement notre ambition pour la MSH, mais aussi le début du commencement de nos difficultés! Car, encore une fois, les sciences "dures" de Paris-Saclay n'éprouvaient pas le besoin de se rapprocher des SHS. Ces sciences ont leur propre grammaire et ont vocation à répondre aux besoins de l'industrie, par la valorisation des résultats de recherche, en se coulant dans la vision orthodoxe de l'économie de marché, actuellement dominante. Dès lors, l'apport des SHS pouvait leur paraître au mieux inutile, au pire contreproductif du fait du regard critique inhérent à la démarche des chercheurs en SHS. Pour m'en tenir à la sociologie du travail que je pratique, il est clair que son approche pouvait amener à questionner les risques d'instrumentalisation de la recherche par le monde industriel, à d'autres fins que le progrès de l'humanité...

\section{《(...) je retiens d'abord l'exercice} maïeutique au sens où il m'a d'abord fallu faire parler les premiers intéressés - les directeurs d'unités de recherche - en plus de faire en sorte qu'ils se parlent davantage, par-delà leur discipline... \)

- En dehors du MIT, aviez-vous en tête des modèles de MSH?

J'avais établi des relations avec d'autres MSH, notamment celle de ParisNord, qui, autant le dire, constitua notre modèle de référence, d'autant plus que nous bénéficions des conseils bienveillants d'Alain Bertho, son directeur. J'ai été aussi très intéressé par la MESH de Lille («E », pour Européenne) que je suis allée visiter. Elle se caractérisait par un rapport très fort avec la région Nord-Pas-de-Calais, ce qui ne m'était pas indifférent au regard de l'exigence de l'inscription territoriale.

Pour en revenir à notre projet de $\mathrm{MSH}$, il se heurtait à une autre difficulté : les divergences entre des chercheurs partisans d'une organisation de la recherche sous l'égide du CNRS et ceux attachés à une 
recherche universitaire, plus individualiste. Pour le dire autrement, le fort engagement du CNRS a pu susciter une certaine défiance de certains.

Autant le reconnaître, je n'avais pas conscience de la réalité des rapports de force qui ont pu se jouer, ni mesuré à quel point, au sein du monde de la recherche, la logique de compétition pouvait l'emporter sur une logique de coopération... La MSH avait beau être une unité de service et, donc, ne pouvoir qu'apporter du mieux, nous nous heurtions à un rejet de la part de certaines composantes disciplinaires.

- La MSH n'en a pas moins passé le cap des cinq ans d'existence. Avec le recul, considérez-vous qu'au final tout était question de temps, pour permettre aux chercheurs des SHS, d'une part, des sciences dites "dures", d'autre part, de mieux se connaître, de surmonter leurs préjugés réciproques?

Je souscris à cette hypothèse, qui permet de comprendre la réussite de la MSH Paris-Saclay dans la durée. Mais au moment où elle a été officiellement créée, en 2015, elle était encore loin de disposer des ressources et des moyens dont elle avait besoin. Au début, je ne disposais que de 10000 euros de crédits alloués par le CNRS, sans le moindre personnel...

\section{"J'estime avoir eu la chance de contribuer à l'émergence de la MSH Paris-Saclay dans une démarche collective avec Stefano Bosi, donc, et André Torre, les deux directeurs adjoints pendant mon mandat $\gg$}

\section{- Comment expliquez-vous cette situation?}

J'y ai vu la confirmation du moindre intérêt du monde scientifique pour la recherche en SHS. Une responsable scientifique à qui je faisais part de mes difficultés à obtenir les moyens pour faire fonctionner «ma » $\mathrm{MSH}$, eut cette réponse qui résumait bien la situation : à la différence des sciences exactes ou du vivant, les sciences de l'Homme et de la Société n'ont pas de prix Nobel ou de Médaille Fields... Ceci, ajouté aux rivalités entre disciplines, j’ai fini par considérer qu'il était temps que je passe la main. 
- Avec le sentiment d'un constat d'échec?

Avec le recul, je considère que mon rôle aura été celui de l'accoucheur. En somme, j’aurais été le Socrate de la MSH Paris-Saclay (rire) !...

- Sans en avoir connu le même sort, tout de même!

Dieu merci, non. Cela tient peut-être à une humanisation des peines! De Socrate, je retiens d'abord l'exercice maïeutique au sens où il m'a d'abord fallu faire parler les premiers intéressés - les directeurs d'unités de recherche - en plus de faire en sorte qu'ils se parlent davantage, pardelà leur discipline...

- Parallele pour parallèle, considériez-vous que, comme dans le cas d'une entreprise innovante, celui qui la crée n'est pas forcément celui à qui reviendra d'en assurer le développement?

Je souscris à ce parallèle, en soulignant également le rôle capital joué par mon successeur Stefano Bosi. Son approche de l'économie l'avait mis au contact des mathématiciens, ce qui est un atout quand on sait la place privilégiée qu'ils occupent au sein de Paris-Saclay. Sa connaissance irremplaçable du monde de la recherche a été un levier crucial pour surmonter les blocages que j'ai rencontrés. Son ouverture aux autres disciplines en SHS a suscité l'adhésion des parties prenantes.

- Manifestement, c'est le sociologue qui s'exprime, de surcroît avec un regard dépourvu d'amertume...

Tout à fait ! Il n'y a aucune raison d'éprouver de l'amertume, encore moins du ressentiment. J'estime avoir eu la chance de contribuer à l'émergence de la MSH Paris-Saclay dans une démarche collective avec Stefano Bosi, donc, et André Torre, les deux directeurs adjoints pendant mon mandat. Le premier a su placer la MSH en orbite avec une capacité de travail bien supérieure à la mienne ! Son propre successeur, André Torre, a su confirmer l'essai par sa vision claire et l'exercice d'une force tranquille. Au final, le trio que nous formions dès le départ a su donner le meilleur à travers un rôle spécifique assumé par chacun au fil du temps. Ensemble, 
nous aurons ainsi participé à la construction d'une institution, avec toute la dimension politique que cela peut revêtir. Les difficultés que nous avons pu rencontrer sont inhérentes à ce genre de projet. Cela étant dit, il y a une personne à laquelle je tiens également à rendre un hommage appuyé, c'est Christine Bénichou, la secrétaire générale de la MSH, qui est arrivée à ce poste en 2016. C'est elle qui a donné à la MSH une existence physique, à travers son implantation au sein de l'ENS Cachan. Elle a ensuite accompagné l'extension des moyens et le recrutement du personnel, permettant ainsi à la $\mathrm{MSH}$ de développer ses missions, y compris au plan éditorial et de la communication (à ce propos, il faut rendre hommage au précieux concours d'Éric Valdenaire).

Les efforts consentis pour créer et développer la MSH auront été si importants qu'on n'aura finalement pas vu le temps passer ! Mais le fait est : la MHS Paris-Saclay a 5 ans ! Elle est désormais bien inscrite dans le paysage de Paris-Saclay et celui de la recherche française en général.

- Dans quelle mesure cette aventure aura-t-elle contribué à modifier votre propre perception de la recherche scientifique et technologique?

Je dois reconnaître que mon propre rapport à cette recherche a évolué et ce, positivement. J'ai été en particulier fasciné par l'univers de la recherche du CEA, qu'il m'a été donné de découvrir, dans le domaine de l'Énergie en particulier. Je l'ai été tout autant par une enquête réalisée sur le Synchrotron, en découvrant notamment l'existence de cette ligne de lumière dédiée aux matériaux anciens étudiés par les historiens et préhistoriens. Une illustration de cette interdisciplinarité forte que nous appelions de nos vœux avec la création de la MSH Paris-Saclay...

- Avec laquelle le lien n'a pas été rompu. Pour preuve, ce workshop que vous avez organisé les 19-20 septembre 2019, avec son soutien...

Effectivement, la MSH Paris-Saclay a soutenu ce workshop autour de Robert Salais. Indépendamment du fait que ce dernier fut mon père spirituel, ce soutien était pleinement justifié : il n'a cessé d'œuvrer à l'interdisciplinarité à partir de l'économie, une démarche plutôt rare chez les économistes. Il a aussi initié un dialogue avec la recherche 
technologique au sein de Paris-Saclay, en proposant, il y a plus de vingt ans, le concept de « science pratique».

Mon lien avec la MSH Paris-Saclay est d'autant moins rompu que je continue par ailleurs à participer à des activités d'expertise dans le cadre d'appels à projets. Et puis, jusqu'en 2018, j'ai été un des codirecteurs de L'Homme \& la Société, l'une des deux revues scientifiques hébergées par la MSH. C'est dire si je ne demanderais qu'à assister aussi à la célébration de ses... 10 ans ! 



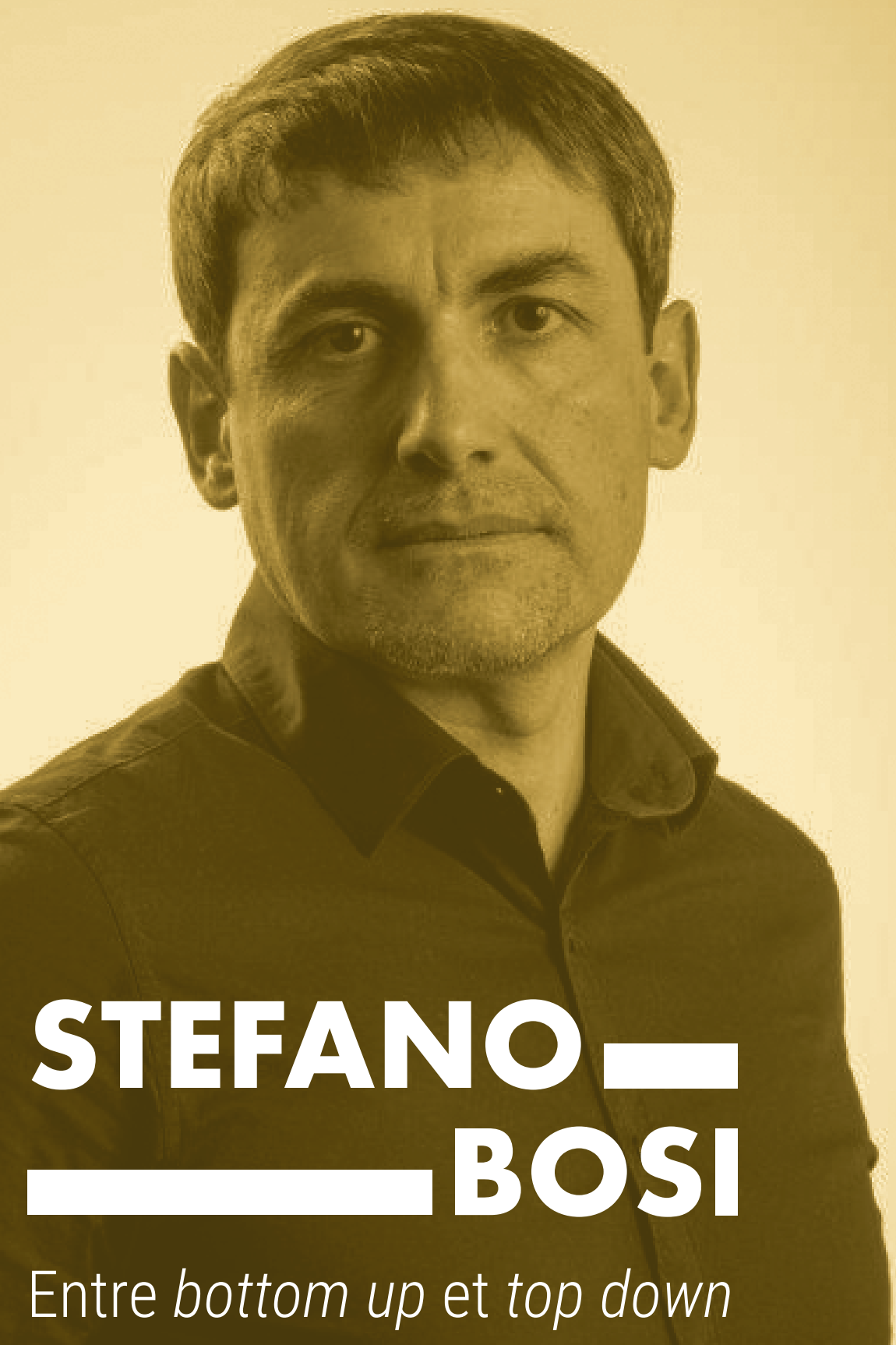




\section{Stefano BOSI}

Vice-Chancelier des Universités de Paris

Ex-directeur de la MSH Paris-Saclay

- Qu'est-ce qui vous a motivé à participer à l'aventure de la MSH ParisSaclay, si ç'en fut une?

Ç’en fut une, effectivement, car il s'agissait ni plus ni moins de poursuivre celle débutée il y a plus de cinquante ans, en 1963, avec la création, au boulevard Raspail, à Paris, de la toute première MSH, à l'initiative de l'historien Fernand Braudel, qui souhaitait par ce moyen promouvoir l'interdisciplinarité entre les SHS. Depuis, d'autres MSH ont vu le jour. Il en existe actuellement une vingtaine. Celle de Paris-Saclay est la dernière création en date. Avant d'en être le directeur, j'ai pris part à la gestation du projet, lequel s'est imposé dès l'instant où a été créée l'Université Paris-Saclay.

- À quel titre aviez-vous pris part à cette gestation?

À l'époque, j'étais directeur adjoint scientifique à l'Institut des sciences humaines et sociales (InSHS) du CNRS - j'avais la responsabilité de la linguistique, de l'économie et des sciences de gestion. Avec Bertrand Jouve, directeur adjoint scientifique en charge des MSH et des IEA (Instituts d'études avancées), nous nous étions accordés sur la pertinence d'adosser une MSH à une université qui visait l'excellence mondiale. Bien évidemment, la décision avait été prise collectivement au sein de l'InSHS, alors dirigé par Patrice Bourdelais. 
Un nom s'était imposé pour en assurer la direction : celui de Claude Didry, sociologue et directeur de recherche au CNRS. Le défi n'était pas mince : il fallait créer la maison de toute pièce, la doter de moyens financiers et humains, impulser une dynamique. Comme pour tout projet de cette envergure, il y eut des difficultés au démarrage. Claude Didry eut le mérite de rédiger un premier projet et de mettre en place un secrétariat général.

- Preuve s'il en était besoin que le concept des MSH avait beau avoir plus de 50 ans d'existence, leur création reste à chaque fois une nouvelle page blanche à écrire...

Absolument. À chaque MSH, son contexte institutionnel, son projet et, donc, ses conditions de création. Il ne suffit pas de transposer un modèle pré-existant. Il faut mobiliser les volontés, séduire les organismes de tutelle - en l'occurrence les universités de Paris-Sud, de Versailles SaintQuentin-en-Yvelines et d'Évry-Val-d'Essonne, l'ENS Cachan, devenue depuis l'ENS Paris-Saclay, et le CNRS.

Patrice Bourdelais m'a demandé si je pouvais m'impliquer en mettant à profit mon expérience de l'InSHS et ma connaissance des MSH, pour contribuer au développement de celle de Paris-Saclay. C'est ainsi que j'en suis devenu le directeur en septembre 2016. La MSH n'avait encore qu'un an et demi d'existence.

\section{《Les SHS étaient bien présentes à Paris- Saclay, mais elles souffraient d'un manque de visibilité, comparées aux sciences exactes comme aux sciences de la vie \)}

- Qu'avez-vous entrepris pour la dynamiser?

D'emblée, il m'a paru indispensable de dresser une cartographie complète des forces SHS en présence à Paris-Saclay. À l'époque, la scission entre deux pôles, l'Université Paris-Saclay et l'Institut polytechnique de Paris, n'était pas encore envisagée. Le champ était donc vaste, incluant les unités de recherche des universités, mais aussi des écoles d'ingénieurs, 
sans oublier HEC. Au total, 37 laboratoires en SHS ont été inventoriés, totalisant près de 1500 chercheurs, dont à peu près 800 doctorants. Soit une force de frappe tout sauf négligeable.

- C'est beaucoup moins que les effectifs des sciences de l'ingénieur ou [sciences] exactes, mais ce n'est effectivement pas négligeable. Vous attendiez-vous à cela?

Non, autant le dire. J'ai été le premier surpris. Les SHS étaient bien présentes à Paris-Saclay, mais elles souffraient d'un manque de visibilité, comparées aux sciences exactes comme aux sciences de la vie. Ce manque de visibilité n'est pas un problème spécifiquement Paris-saclaysien. Il concerne l'ensemble des SHS françaises. On peut le regretter, mais on peut aussi y voir un potentiel. 1500 personnes rien qu'à Paris-Saclay, ce n'est pas rien! Il nous fallait juste catalyser cette richesse, en lui donnant les moyens de s'exprimer à l'international.

- Les financements étaient-ils à la bauteur de vos attentes?

Oui, c'est le moins qu'on puisse dire. Toutes nos tutelles ont tenu leurs engagements. Le travail de cartographie a, semble-t-il, contribué à susciter leur adhésion, de même qu'à faciliter la définition des axes thématiques.

Naturellement, je n'étais pas seul pour mener tout cela. J'ai pu m'appuyer sur le Bureau que j'ai mis en place et un Conseil scientifique, dont j'ai finalisé la composition en y faisant entrer des pointures reconnues dans leur domaine en SHS, bien sûr, mais aussi en sciences “exactes", pour traduire notre volonté d'une interdisciplinarité forte. Ce conseil scientifique participe pleinement à la vie de la MSH en étant force de propositions. Durant mes années à sa direction, les effectifs de permanents sont passés de deux à six personnes.

- Comment avez-vous pu identifier vos axes thématiques?

Il fallait se garder d'être hors-sol, d'enclencher une dynamique déconnectée de ce qui se faisait concrètement dans les laboratoires. Nous avons donc procédé à une double logique. Bottom up, d'abord, consistant à regarder ce sur quoi les SHS de Paris-Saclay étaient mobilisées - nous disposions pour cela des mots clés par lesquels les chercheurs avaient 
caractérisé à notre demande leurs thématiques de recherche à l'occasion du travail de cartographie. Top down, ensuite, consistant à définir des choix stratégiques avec les établissements de tutelle. Deux opportunités se présentaient de toute évidence. La première : développer un axe autour des enjeux des transitions écologique et énergétique : de nombreux chercheurs de Paris-Saclay, travaillaient dessus, tant du côté des SHS que des sciences exactes et des sciences de la vie - l'UVSQ en particulier était bien positionnée internationalement en climatologie.

La seconde opportunité était de traiter d'une autre transition : la transition numérique, liée au Big Data et à l'IA (Intelligence articielle). Là encore, Paris-Saclay concentre des moyens parmi les plus importants en Europe, tant du côté des sciences dures, grâce notamment à la présence de l'Inria et d'autres laboratoires, que de celui des SHS.

Ces deux opportunités nous ont confortés dans l'idée de faire de la MSH un lieu de renouvellement des questionnements des SHS, d'engager cellesci résolument dans des enjeux d'avenir, bref, de créer les SHS du futur.

Sans vouloir verser dans une vision catastrophiste, il faut quand même rappeler que nous assistons à la sixième extinction massive d'espèces, et ce sur fond d'augmentation de la température moyenne du globe. À quoi s'ajoute cette transition numérique qui est en train de révolutionner nos modes de vie en société. Les SHS doivent se prononcer sur ces enjeux, nous éclairer, non pas en étant seulement en soutien des sciences exactes ou de la vie, mais en prenant l'initiative de projets de recherche interdisciplinaire avec elles.

- Comment vous y êtes-vous pris pour traduire cette interdisciplinarité dans les faits?

Naturellement, nous nous sommes gardés de contraindre les chercheurs. Nous les y avons plutôt incités à travers des appels à projets, non sans toutefois imposer à chaque fois des conditions strictes. Les projets qui nous étaient soumis devaient en effet s'inscrire dans l'un ou l'autre des axes stratégiques que je viens d'évoquer. Ils devaient, en outre, être à la fois interdisciplinaires et inter-établissements (la MSH Paris-Saclay a aussi vocation à contribuer à renforcer les liens entre universités et 
grandes écoles). Dès lors qu'un projet cochait les trois cases, il pouvait bénéficier d'un financement, a fortiori s'il était original, porté par une équipe solide et motivée.

Plusieurs catégories d'appels à projets ont été mis en place, reconduits annuellement. D’une part, l'appel à projets Émergence qui, comme son nom l'indique, a vocation à amorcer de nouvelles problématiques de recherche. D'autre part, des appels à projets dit «Maturation », destinés à accompagner des projets demandant à être approfondis et à changer d'échelle, et bénéficiant pour cela d'un soutien plus important. À quoi s'ajoutent les appels à workshops ou à séminaires.

\section{"Ces deux opportunités nous ont confortés dans l'idée de faire de la MSH un lieu de renouvellement des questionnements des SHS, d'engager celles-ci résolument dans des enjeux d'avenir, bref, de créer les SHS du futur i)}

- Dans quelle mesure les chercheurs en SHS de Paris-Saclay se sont-ils appropriés la MSH?

À l'évidence, la MSH correspondait à leurs attentes, à en juger par le nombre de projets qu'ils ont soumis dès les premiers appels à projets. Pas moins de 75 conférences et workshops interdisciplinaires ont été organisés durant ma première année à sa direction. Du jamais vu dans le champ des SHS de Paris-Saclay. On peut donc dire que la «mayonnaise a pris ». Ce qu'ont reconnu les tutelles elles-mêmes. Preuve s'il en était besoin qu'on pouvait faire de très belles choses à Paris-Saclay, y compris en SHS. Le succès a été tel que, malheureusement ou heureusement, on m'a demandé de prendre d'autres responsabilités - en février 2018, j’ai été nommé Vice-Chancelier des Universités de Paris.

- Aviez-vous procédé à ce qu'il est convenu d'appeler du benchmark, si tant est que ce terme soit en usage dans le champ des SHS ?

Avant de prendre la direction de celle de Paris-Saclay, je connaissais bien le réseau des MSH. Ensuite, une fois directeur, j'ai beaucoup 
échangé avec mes homologues, non sans cependant revendiquer une particularité pour la MSH Paris-Saclay : cette interdisciplinarité des SHS avec les sciences exactes ou de la vie. Comment pouvait-il en être autrement ? Paris-Saclay représente un environnement exceptionnel, qui offre l'opportunité d'instaurer un vrai dialogue entre un large spectre de sciences, qui n'ont pas encore autant l'occasion de travailler ensemble. Cette interdisciplinarité forte devait être notre marque de fabrique. Pour autant, nous ne nous sommes heurtés à aucune résistance. Au contraire, le président du Réseau national des $\mathrm{MSH}$, qui n'était autre que Bertrand Jouve, nous a fait pleinement confiance. Il a conçu que ce pouvait être une valeur ajoutée par rapport à la vision courante des MSH.

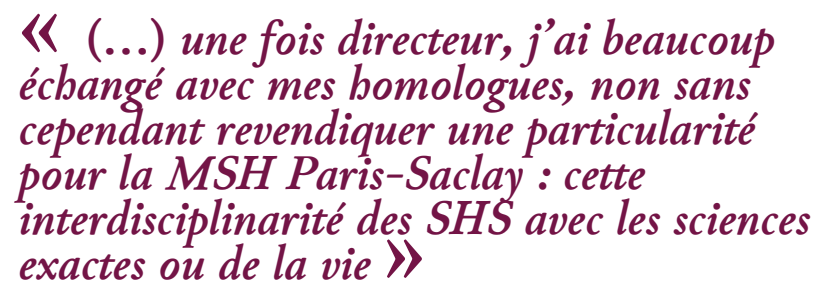

- Dans quelle mesure le fait que la direction ait été confiée à un européen francophone d'origine transalpine a-t-elle pu contribuer à cette réussite?

En plus d'être italien, j'ai fait des études dans un autre pays européen, la Belgique en l'occurrence. Forcément, cela incline à adopter une vision d'emblée plus internationale, à faire du benchmark, pour reprendre votre mot, à être attentif à ce que les autres font dans le domaine des SHS. Certes, on ne trouve pas de MSH ailleurs - c'est, faut-il le rappeler, un concept spécifiquement français. Pour autant, les SHS d'autres pays n'en pratiquent pas moins de longue date une interdisciplinarité forte. Qu'on songe aux SHS anglo-saxonnes. Force est de constater aussi que ce sont précisément ces recherches-là, qui reçoivent un grand écho dans le monde de la recherche ou encore des médias. Personnellement, ce sont elles qui me séduisent le plus et m'ont convaincu non pas tant de créer autre chose qu'une MHS, mais de faire de celle de Paris-Saclay un lieu de modernisation et d'internationalisation des SHS françaises. 
- Continuez-vous néanmoins à suivre l'actualitéde la MSH de Paris-Saclay? Oui, bien sûr. Il me faut juste veiller à éviter tout conflit d'intérêt avec mes nouvelles responsabilités! L'évolution que la MSH a suivie depuis est plus que positive. Mon successeur, André Torre, a su entretenir la dynamique en étoffant les appels à projets : aux appels à projets Émergence et Maturation, il a ajouté des appels à projets Excellence. Le nombre de conférences et de workshops a continué à se développer à un haut niveau : il y en eut autant davantage l'année correspondant à son entrée en fonction, que durant la précédente.

Lui et moi avons en commun d'être des économistes et de manifester le même intérêt pour l'interdisciplinarité. Certes, nos approches sont différentes, mais chacune incline à dialoguer avec d'autres disciplines : les mathématiques, dans mon cas, la sociologie et la géographie, dans le sien.

- Dans quelle mesure l'expérience de la MSH vous a-t-elle permis de questionner votre propre pratique de l'économie?

D'un point de vue disciplinaire, je suis un économiste qui mobilise les outils mathématiques, sans être pour autant mathématicien de formation. Je relève donc plus des SHS que des sciences exactes, mais avec une forte prédisposition à une interdisciplinarité des premières vers les secondes.

Cela étant dit, j'ai toujours eu beaucoup de curiosité pour d'autres disciplines. Une curiosité que mes années au sein de l'InSHS - j'y suis resté quatre ans et demi - m'a permis d'exprimer encore davantage. Tous les jours, je me retrouvais à discuter de problématiques de disciplines apparemment très éloignées les unes des autres : la linguistique et les sciences de gestion, donc, mais aussi celles qui sont couvertes par les autres directeurs adjoints avec lesquels j'étais aussi en interaction. Bref, le CNRS m'a permis d'acquérir un regard vraiment interdisciplinaire, que mon expérience de la MSH de Paris-Saclay m'aura donné l'occasion de faire fructifier et ce, pour ma plus grande joie. 



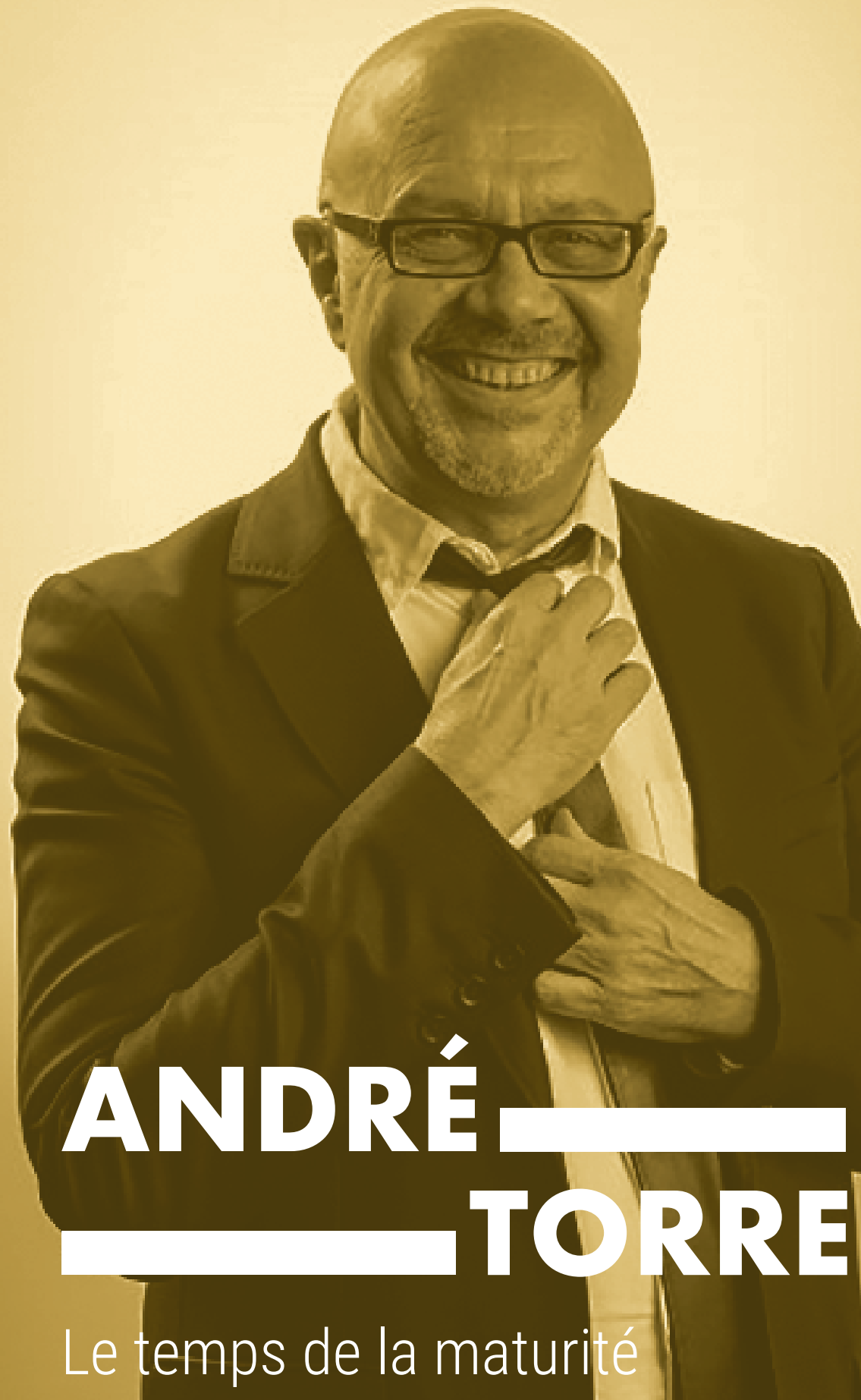




\section{André TORRE \\ Directeur de recherche Inra/AgroParisTech, à l'UMR SAD-APT (UMR 1048) Directeur de la MSH Paris-Saclay}

- Si vous deviez rappeler, pour commencer, ce qu'est une Maison des Sciences de l'Homme?

C'est effectivement important de le rappeler, car ces structures ne sont pas toujours bien connues du grand public. Quelqu'un me demandait récemment sur le ton de la blague si c'était une réponse au phénomène $\# M e T o o$, autrement dit, si ces maisons avaient vocation à prendre la défense des hommes... Il ne s'agit là que d'une anecdote, mais qui dit quelque chose de l'air du temps et de la notoriété des MSH en dehors du monde universitaire. C'est bien évidemment de l'Homme avec un grand $\mathrm{H}$ dont il est question. Une MSH fédère les chercheurs qui travaillent sur les humains en général, hommes et femmes, donc.

Historiquement, ces maisons sont relativement anciennes. Elles ont été créés à partir des années 1950, à l'initiative de l'bistorien Fernand Braudel, pour promouvoir les sciences sociales à travers des programmes interdisciplinaires impliquant des chercheurs de toutes provenances.

Au total, plus d'une vingtaine de MSH ont été créées, que le CNRS a, ces dernières années, contribué à harmoniser du point de vue des statuts et des missions. Elles constituent aujourd'hui le principal levier d'action de son Institut des Sciences Humaines et Sociales (InSHS), lequel vise à promouvoir une recherche de haut niveau dans ces sciences, à l'image de ce qui peut se faire dans les sciences "dures", de l'ingénieur ou encore du vivant. 
- Qu'est-ce que cela dit de la vision des SHS ainsi promues? Sont-elles appelées à privilégier une approche plus quantitative, sinon modélisatrice, voire technologique, comme ces sciences dites "dures" qu'incarne de prime abord le CNRS ?

Comparativement aux sciences "dures" comme d'ailleurs [aux sciences] du vivant, les SHS ont longtemps paru davantage émiettées, parcellisées, entre de petites équipes et des laboratoires moins bien dotés. C'était particulièrement vrai des sciences davantage tournées vers les « humanités ». L'ambition du CNRS a donc été de leur donner une plus grande visibilité, en fédérant des équipes autour de grandes thématiques, mais aussi d'infrastructures et d'équipements de recherche... Donc, pas d'exclusive, nous accueillons aussi bien les recherches quantitatives que celles d'essences plus qualitatives ou spéculatives.

- De quels équipements et infrastructures peut-il donc s'agir dans le cas des SHS ?

L'exemple le plus évident, ce sont les plateformes permettant de gérer et traiter les bases de données utilisées dans les SHS : il est important pour les chercheurs de pouvoir disposer de données fiables pour mener à bien leur travail, mais aussi d'être en mesure de protéger ou de diffuser leurs propres données. En la matière, nous ne partons pas de rien. Il existe aujourd'hui des TGIR (Très Grandes Infrastructures de Recherche), qui permettent d'héberger les données des chercheurs, selon différents niveaux de protection, mais aussi de leur faciliter l'accès à des bases de données fiables. Notre intention est d'accompagner cette évolution.

- Si les MSH sont des réalités anciennes, la vôtre est de création récente...

Oui, et c'est la première chose qu'il convient de rappeler. La MSH ParisSaclay a été créée en 2015. J'ajoute que notre MSH n'est véritablement en état de fonctionner que depuis la mi-2016. Cela ne l'a pas empêchée d'avancer à marche forcée, sous la houlette de mon prédécesseur, Stefano Bosi, et grâce au soutien de la Comue et du CNRS, qui ont mis à disposition les financements et les postes nécessaires. La dynamique aura été si bien enclenchée que notre MSH a déjà lancé pas moins de 90 actions rien qu'en 2018 et en aura réalisé plus d'une centaine à la fin 2019 - entre projets de recherche, séminaires et workshops. 
- Combien de chercheurs fédère-t-elle?

Autant le reconnaître, au moment où la MSH Paris-Saclay a été créée, nous l'ignorions! Nous avons donc procédé à un recensement, en passant en revue les laboratoires relevant des établissements de recherche du campus Paris-Saclay. Avec Stefano Bosi, Claude Didry et quelques autres, nous nous réunissions à AgroParisTech, rue Claude Bernard, chacun indiquant les laboratoires qu'il connaissait. Petit à petit, nous avons établi une première liste. À chaque laboratoire identifié, nous avons adressé un formulaire pour qu'il nous précise sa composition. Puis nous avons traité les données avec un tableur Excel pour comptabiliser les effectifs discipline par discipline. Ça s'est fait aussi simplement que cela. Ainsi, je suis en mesure de vous dire que la MSH de Paris-Saclay compte actuellement plus de 1200 enseignements-chercheurs. Si, donc, les sciences "dures", exactes ou de l'ingénieur dominent à Paris-Saclay, on ne peut pas dire que les SHS y sont quantité négligeable.

- Quelles disciplines dominent au sein de ces SHS?

Grosso modo, nos chercheurs sont pour un quart d'entre eux des économistes, pour un autre quart des sociologues, pour un troisième, des juristes. Toutes les autres SHS pèsent un dernier quart.

\section{《Notre intention est de placer la MSH Paris-Saclay au cour de ce cluster, certainement pas d'en faire un îlot, qui cultiverait sa singularité $\gg$}

- Où est implantée votre $M S H$ ?

Pour l'heure, elle est hébergée dans les locaux actuels de l'ENS ParisSaclay, à Cachan, donc. Mais, elle a vocation à rejoindre et ce, dès le printemps 2020, les nouveaux locaux de cette école, sur le plateau de Saclay. Nous nous installerons dans le magnifique bâtiment de Renzo Piano. C'est dire si nous sommes plus qu'heureux de travailler dans un pareil endroit où nous pourrons aussi disposer de l'amphithéâtre de l'école et bénéficier des activités autres que scientifiques qui y seront programmées. 
- Comment apprébendez-vous votre présence au milieu d'autres établissements d'enseignement supérieur et de recherche, tournés davantage vers ces sciences?

Notre intention est de placer la MSH Paris-Saclay au cœur de ce cluster, certainement pas d'en faire un îlot, qui cultiverait sa singularité. Nous travaillons donc déjà avec des chercheurs en sciences "dures" ou [sciences] de l'ingénieur, et menons de nombreux projets avec eux. Mais ce n'est pas tout : nous avons aussi conscience que cet environnement compte de nombreuses entreprises et une société civile dynamique. Nous souhaitons donc échanger avec elles, au travers de projets que nous co-construirons. C'est ainsi que l'an dernier, nous avons participé à la réponse à l'appel à manifestation d'intérêt TIGA (Territoire d'Innovation - Grande Ambition) et accueilli les workshops préparatoires.

\section{《 (...) nous avons récemment proposé une inflexion consistant à encourager des projets interdisciplinaires de plus grande ambition, impulsés par des équipes de SHS (...) \)}

- Revenons à la visée interdisciplinaire de votre MSH. Comment procédezvous concrètement pour fédérer des chercheurs en SHS?

Nous les mobilisons au travers d'appels d'offres qui financent, selon le cas, des projets de recherche, des séminaires ou des workshops, la condition étant que l'une ou l'autre de ces actions soit interdisciplinaire mais aussi inter-institutionnelle, autrement dit, qu'elle mobilise différentes disciplines et laboratoires appartenant à différents organismes. Pour faciliter la vie de nos chercheurs, leur permettre de se consacrer à ce qu'ils savent faire le mieux (la recherche !), nous assurons la logistique et l’organisation, jusqu'à la commande des petits fours si besoin !

Nous avons, par ailleurs, mis en place un pôle de communication, avec un responsable et une apprentie, qui prend en charge la diffusion de l'information (au travers de supports et des réseaux sociaux). Nous avons, d'autre part, constitué un pôle édition : en plus d'héberger deux revues, nous éditons des ouvrages - quatre à ce jour, issus de séminaires - à notre compte, ou dans le cadre d'une maison d'édition créée avec les 
MSH de Paris Nord et de Nanterre (MSHA, pour les MHS associées, qui recourt à OpenEdition).

Enfin, toujours dans ce souci de favoriser la collaboration interdisciplinaire et entre sites, nous réalisons également un annuaire de nos chercheurs, identifiables par mots clés - mis en ligne depuis l'hiver dernier, il connaît un grand succès et leur permet de se retrouver en fonction de leurs centres d'intérêts.

- De quels effectifs dispose votre MSH pour mener à bien ces diverses activités?

De sept personnes, dont deux en CDD, le restant étant des postes mis à disposition par le CNRS ou la Comue. Pour autant, nous ne souhaitons pas nous limiter à l'interdisciplinarité au sein des SHS. Nous nous sommes assignés une deuxième mission, dont je me demande comment nous ne pourrions pas l'assumer : être à l'interface avec les autres sciences, "dures" ou du vivant. Soit ce que, à l'Inra, nous appelons une interdisciplinarité «élargie ».

- Soit. Mais, cela fait des années qu'on parle d'interdisciplinarité. Qu'en estil vraiment? Les chercheurs en SHS s'y prêtent-ils facilement?

Je ferai deux remarques, l'une sur la situation actuelle, l'autre sur notre ambition.

La situation actuelle, d'abord. Le moins qu'on puisse dire est qu'elle est variable. Des équipes et des laboratoires ont déjà une expérience ancienne de l'interdisciplinarité. C'est le cas des équipes des laboratoires du CEA, de l'Inra, d'AgroParisTech, des spécialistes du patrimoine ou des sciences du vivant, ... A contrario, des équipes ou laboratoires sont davantage spécialisés dans un domaine ; leurs chercheurs n'ont pas forcément l'habitude de travailler avec des chercheurs d'autres disciplines. L'interdisciplinarité est donc pour eux un challenge et requiert du temps et un minimum d'apprentissage. Sans compter que certains n'y voient pas d'une nécessité immédiate. De fait, tous les chercheurs en SHS n'ont pas besoin d'interagir avec des chercheurs des sciences "dures" ou du vivant pour faire de la recherche de qualité.

Quoi qu'il en soit, et j'en viens à l'autre aspect de ma réponse, 
notre volonté est bien de développer et de promouvoir les projets qui croisent davantage les SHS avec d'autres sciences. Aujourd'hui encore, les projets proposés en ce sens proviennent souvent des mêmes groupes de chercheurs. C'est bien, mais il faut voir plus loin pour des projets plus ambitieux, pilotés par les SHS et impliquant des sciences dites "dures".

C'est pourquoi nous avons récemment proposé une inflexion consistant à encourager des projets interdisciplinaires de plus grande ambition, impulsés par des équipes de SHS et non, comme cela se passe le plus souvent, par des équipes de sciences "dures" ou du vivant. Notre volonté est d'impliquer davantage ces derniers dans de l'interdisciplinarité, de surcroît élargie. Concrètement, chaque année, nous comptons impulser un ou deux grands projets de recherche sur des thématiques d'actualité, et porté(s) par une équipe dirigée par des chercheurs en SHS. Il s'agit des projets Excellence, dont le premier, «Terribio », démarre maintenant, avec un financement important sur un an et demi, et après une intense compétition ayant impliqué onze candidats départagés par des experts de haut niveau et les membres de notre Conseil scientifique.

\section{- Quelle est votre propre pratique de l'interdisciplinarité ?}

Étant directeur de recherche à l'Inra, je la pratique au quotidien et ce, de longue date. Mon laboratoire, SAD-APT (pour Sciences pour l'action et le développement - Activités, produits, territoires), est une UMR Inra/ AgroParisTech. Il est par définition interdisciplinaire et bien au-delà des SHS, puisqu'il compte des chercheurs en sciences du vivant. Dès la fin des années 1990, j’ai dirigé plusieurs grands programmes pluridisciplinaires de recherche, financés par l'ANR ou l'Europe et requérant le concours d'économistes, mais aussi de sociologues, de géographes, de politistes, de spécialistes de l'aménagement, d'agronomes, etc. Mais je dois reconnaître que ce n'est qu'au prix d'un long apprentissage que je suis vraiment parvenu à faire ce type de recherche...

- Comment composez-vous avec les éventuelles réticences des chercheurs à interagir avec le monde de l'entreprise en particulier, si tant est que ces réticences soient toujours d'actualité ? Qu'en est-il de la valorisation scientifique et des rapports des chercheurs en SHS à l'égard des entreprises? 
Beaucoup reste à faire pour rapprocher ces deux mondes. Mais tout dépend, encore une fois, des disciplines et des laboratoires, et il n'est pas question de forcer qui que ce soit. Certaines équipes ont l'expérience et la culture de la relation partenariale avec l'entreprise. C'est souvent le cas de celles des grandes écoles. Reconnaissons que pour d'autres disciplines ou équipes (je pense par exemple à nos spécialistes de musicologie), les interactions ne vont pas de soi, même avec la meilleure volonté du monde, et que les tropismes disciplinaires doivent être respectés. Mais on a souvent de très bonnes surprises!

\section{$\ll(. .$.$) je trouve proprement passionnante$ l'aventure consistant à créer une $M S H$, et celle-ci en particulier : en plus de couvrir un large spectre de SHS, elle s'inscrit dans la dynamique de Paris-Saclay, un cluster technologique de première importance \}}

- On vous sent très motivé et même enthousiaste pour l'avenir de la MSH?

Comment ne pas l'être ? Au plan scientifique, nous avons tout pour réussir. Le seul bémol, j’y reviens, concerne les conditions d'accessibilité du plateau de Saclay, mais aussi des déplacements au sein de Paris-Saclay. Une situation qui pourrait réduire les avantages d'une implantation sur place. Rappelons qu'en plus d'établissements présents sur le plateau de Saclay, elle comprend les universités d'Évry, de Versailles Saint-Quentin-en-Yvelines et de ParisSud, dont la proximité géographique est toute relative.

- On sent que c'est de nouveau l'économiste de l'innovation territoriale qui parle...

Oui (rire). Je crains que vous ne m'ayez démasqué...

- On comprend à quel point vous étiez prédisposé à prendre la direction de la MSH de Paris-Saclay, et à y déployer ces programmes de recherche interdisciplinaire. Mais qu'est-ce qui a motivé votre décision de vous porter candidat? 
Ma motivation a été double. D’abord, cela fait longtemps que je m'intéresse au projet du cluster Paris-Saclay. Certes, je le reconnais, mon intérêt n'était pas dénué de scepticisme. En tant que chercheur dans le domaine de l'économie spatiale, je m'interrogeais quant à savoir si c'était un "vrai" cluster. Mes doutes ne sont pas totalement levés. Mais à partir du moment où il a été décidé que l'Inra et AgroParisTech rejoindraient le plateau de Saclay - à l'époque, j'étais directeur adjoint de l'UMR SADAPT et animais l'équipe Proximités - j'ai considéré qu'on ne pouvait pas y aller à reculons, que malgré mon scepticisme, je devais accompagner mes collègues dans le transfert de notre établissement. Bref, en tant que directeur d'équipe, j’ai estimé devoir prendre mes responsabilités. En 2015, j’ai donc participé aux réunions destinées à la constitution du département des SHS de l'Université Paris-Saclay et de la MSH (les choses se sont faites concomitamment). Au moment de choisir entre le département et la MSH, j’ai finalement opté pour celle-ci ; je suis davantage chercheur qu'enseignant, même si j'ai beaucoup enseigné au cours de ma carrière.

Ensuite, quand la MSH a été effectivement créée, Claude Didry en a pris la direction, et nommé deux directeurs adjoints : Stefano Bosi et moi-même. L'année suivante, il démissionnait ; Stefano Bosi, qui lui a succédé, a alors constitué un bureau que j'ai intégré, et il a vraiment lancé la maison, avec beaucoup de talent. Les choses auraient pu se poursuivre ainsi jusqu'à la fin du premier programme quinquennal. Seulement, Stefano a été nommé Vice-chancelier des Universités de Paris. Il m’a paru logique et légitime - j'étais déjà responsable de l'axe Environnement, santé et territoire de la MSH - de me porter candidat à sa direction.

$\mathrm{Au}$-delà de cela, je trouve proprement passionnante l'aventure consistant à créer une $\mathrm{MSH}$, et celle-ci en particulier : en plus de couvrir un large spectre de SHS, elle s'inscrit dans la dynamique de Paris-Saclay, un cluster technologique de première importance. J'avais le sentiment de pouvoir faire profiter de mon expertise dans le domaine des systèmes d'innovation locaux et de mon expérience de l'inter- et de la pluridisciplinarité. La pratiquant au quotidien, j'en connais les avantages, mais aussi les écueils. 
Et, pourquoi ne pas le dire, j'éprouve du bonheur à pouvoir encore à mon âge (62 ans) découvrir des travaux et des disciplines dont j'ai encore tout à connaitre. Une de nos équipes travaille sur les musiques de sortie de guerre! Qu'est-ce que l'économiste a bien pouvoir à dire à ce sujet ?! Et sur "Genre et monde carcéral », autre thématique de recherche interdisciplinaire ? Rien non plus, et c'est tant mieux : j'ai tout à apprendre ! 



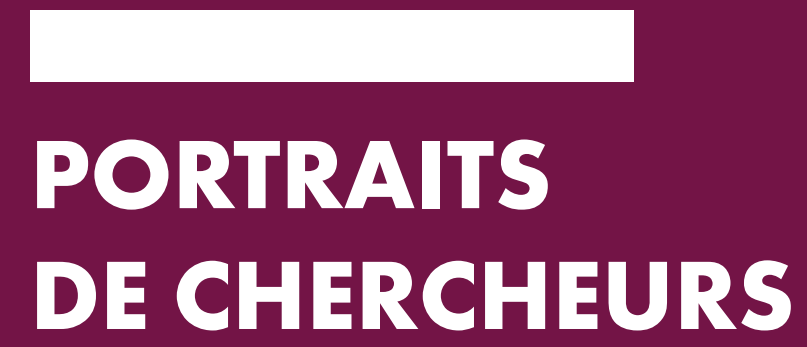




Maître de conférences à Télécom Paris et chercheur à l'i3 (Institut interdisciplinaire de l'innovation ; UMR 9217 ; X, Mines ParisTech, Télécom Paris, CNRS), Antonio Casilli aborde les enjeux du numérique en sociologue. À la suite de projets de recherche qu'il a coordonnés sur les réseaux sociaux en ligne, la santé et la vie privée, il s'est intéressé au « travail du clic " (digital labor) promu par les plateformes numériques.

Lauréat d'un appel à workshops en 2017, il a été la même année lauréat d'un appel à projets Maturation avec le projet DipLab (pour Digital Platform Labor). Lequel vise à rendre visible et organiser le micro-travail à l'œuvre sur les plateformes numériques, à partir d'approches interdisciplinaires. 


\section{Antonio CASILLI \\ Maître de conférences à TélécomParis Chercheur à l'i3-DSES (UMR 9217)}

- Pour commencer, pouvez-vous rappeler comment vous en êtes venu non seulement à vous intéresser au "digital labor» des plateformes numériques, mais encore à pénétrer cet univers?

Dans ma vie précédente, avant mon arrivée en France et mon doctorat, j'étais économiste... et italien. Ce n'est pas une blague, dans la mesure où je suis issu d'une université (la Bocconi de Milan) très centrée sur l'économie politique, mais j'étais aussi un auteur au sein de cette mouvance que l'on appelle aujourd'hui l'Italian Theory, une école de pensée qui analyse les modalités de production de valeur en dehors des contextes productifs classiques. Par-delà les usines et les bureaux, où est-ce qu'on produit la valeur ? Dans les foyers, grâce au travail du care des femmes et des aidants. Dans les universités, où les étudiants sont les cobayes d'un marché du travail de plus en plus dérégulé. Dans les lieux de consommation, où les consommateurs sont désormais appelés à réaliser des gestes productifs comme scanner leurs propres produits, acheter leurs propres billets, monter leurs propres meubles. Et, depuis l'arrivée d'Internet, nos propres équipements nous poussent à réaliser des " tâches informationnelles » qui produisent des données et, donc, de la valeur pour les grandes plateformes numériques. C'est comme ça que le digital labor s'est imposé, sans rupture de continuité, sur ma production plus récente, à partir du début des années 2010. 


\section{$\ll(. .$.$) ce projet vise à comprendre le$ phénomène du micro-travail à l'cuvre sur les plateformes numériques, soit des services numériques d'allocation massive de petites tâches standardisées à des foules de prestataires $\gg$}

- En quoi l'apport de la MSH a-t-il été utile à l'approfondissement de vos travaux de recherche sur ce thème $d u$ "digital labor »?

En premier lieu, la MSH m'a permis d'organiser en décembre 2017, avec Paola Tubaro, du Laboratoire de recherche en informatique (LRI, CNRS/Université Paris-Sud), une conférence sur les enjeux éthiques et déontologiques de la recherche sur les réseaux sociaux ( Recent Ethical Challenges in Social Network Analysis»). Le projet de cette conférence avait été lauréat d'un appel à workshops lancé quelques mois plus tôt.

Ensuite, la MSH nous aura permis, Paola et moi, de suppléer, au travers d'un appel à projets Maturation, lancé la même année, l'absence des financements dont nous avions besoin pour mener à bien le projet DiPLab (pour Digital Plateform Labor). Pour mémoire, ce projet vise à comprendre le phénomène du micro-travail à l'œuvre sur les plateformes numériques, soit des services numériques d'allocation massive de petites tâches standardisées à des foules de prestataires. À terme, ce même projet visait à promouvoir des formes équitables et éthiques d'organisation du travail sur les plateformes numériques.

Les premiers partenaires potentiels que nous avions sollicités n'avaient pas vu d'emblée l'intérêt d'étudier le travail du clic sur les plateformes. $\mathrm{La} \mathrm{MSH}$, si. Son soutien a suffi à convaincre une organisation syndicale (FO) et une agence étatique (France Stratégie) à rejoindre le projet, pour en financer d'autres volets. En ce sens, la MSH a joué le rôle de seed investment (investissement d'amorçage). DipLab a connu depuis plusieurs prolongements : il a fait l'objet d'un rapport final qui a eu un grand écho dans la presse nationale, et d'une importante conférence finale organisée conjointement avec France Stratégie, en juin 2019. 


\section{- Parleriez-vous d'effet levier?}

Bien plus que d'un effet levier : grâce aux données que nous avons pu collecter et aux résultats que nous avons obtenus, nous avons bénéficié de ce qu'on peut appeler un "effet ascenseur ", au sens où les projets ont pu s'enchaîner en gagnant en ampleur et/ou en abordant d'autres aspects du digital labor. À partir du workshop, nous avons pu faire émerger une recherche au travers des autres appels à projets dont nous avons été lauréats, et, enfin, accéder à d'autres appels à projets, ANR, pour notre projet HUSH ( « La chaîne d'approvisionnement humaine derrière les technologies intelligentes ») et européens (pour notre projet SWIRL sur les "Slash workers »). Il s'agit de recherches traitant de sujets connexes. Dans ces différents cas, l'affichage du soutien de la MSH dans nos réponses à ces appels à projet a sans aucun doute pesé dans la décision des jurys.

- Un mot sur l'interdisciplinarité promue par la MSH, avec, pour particularité, de favoriser le dialogue entre les SHS, mais aussi entre celles-ci et les autres sciences (exactes, de la vie...)

En effet, il s'agit d'une interdisciplinarité forte et en cela, elle s'accorde bien avec ma propre sensibilité et pratique de la recherche. Cela fait au moins une vingtaine d'années, que je pratique cette interdisciplinarité, sinon transdisciplinarité, mais encore que je théorise à son sujet. Pour mémoire, j’ai fait ma thèse de doctorat au laboratoire Edgar Morin, l'Institut interdisciplinaire d'anthropologie du contemporain (IIAC). J'ai depuis publié plusieurs articles sur ce thème, et lui ai consacré un séminaire.

Je travaille sur les technologies numériques et leur impact sur la société. Il s'agit là d'« objets à multiples entrées ", qui demandent à chaque fois des compétences qui sont autant issues des SHS que des sciences exactes. L'interdisciplinarité que promeut la MSH est d'autant plus adaptée qu'elle va de pair avec une volonté de rapprocher non seulement des équipes au sein d'un même établissement de recherche, mais encore de différents établissements. Concrètement, dans le cas du projet DipLab, elle a permis d'instaurer une collaboration entre le département de Sciences économiques et sociales de Télécom Paris et 
le Laboratoire de recherche en informatique (LRI) du CNRS, et ainsi de stimuler des méthodes originales sur des sujets à la frontière de différentes disciplines. Non seulement je ressors conforté dans l'idée que la transdisciplinarité est une perspective intéressante et une démarche praticable, mais encore qu'elle est nécessaire pour traiter des enjeux des technologies avancées.

- Une autre particularité de la MSH Paris-Saclay est d'assumer son inscription dans un écosystème territorialisé. En quoi cela a-t-il joué favorablement dans le cas de vos projets, quand bien même ces plateformes numériques apparaissent de prime abord comme étant hors-sol?

Je ne suis pas sûr que cet ancrage territorial de la MSH en soit une spécificité. Je connais d'autres exemples de MSH qui peuvent revendiquer tout autant un tel ancrage - je pense en particulier à la MSH Paris-Nord. Cela étant dit, l'ancrage territorial de celle de Paris-Saclay s'est révélé un atout au moment où la Comue initiale de Paris-Saclay a finalement éclaté en deux Comue, l'Université Paris-Saclay, d'une part, l'Institut Polytechnique de Paris, d'autre part. Le fait de se définir davantage en rapport à un territoire qu'en référence à une Comue a quelque peu permis d'atténuer la complexité née de la situation nouvelle. Pour ce qui me concerne, Télécom Paris a eu beau avoir rejoint la seconde Comue, j'ai pu continuer à mener des projets impliquant des collègues dont l'établissement se situait de l'autre côté de la «frontière ». Pour le dire autrement, la MSH aura permis de maintenir les liens, en atténuant les effets de la séparation.

- Venons-en à votre ouvrage, En attendant les robots (Éditions du Seuil, 2019) dans lequel vous décrivez la réalité du digital labor : l'exploitation des petites mains de l'intelligence " artificielle » par les plateformes numériques. Cet ouvrage a eu un large écho dans les médias et dans le monde de la recherche. En quoi est-il redevable aux projets menés avec l'appui de la MSH?

Cet ouvrage s'emploie en effet à révéler l'illusion de l'automation intelligente, en mettant en évidence les myriades de tâcherons du clic soumis au management algorithmique de plateformes en passe de 
reconfigurer et de précariser le travail humain. Il est le résultat d'un travail entamé il y a plusieurs années et lié à une étape importante dans la carrière d'un chercheur en SHS - je veux parler de la soutenance de l'HDR (Habilitation à diriger les recherches) que, pour ma part, j'ai soutenue à l'Université Paris-Dauphine. La rédaction de mon mémoire a commencé au moment où j'entamais ma collaboration avec la $\mathrm{MSH}$, au travers du projet DipLab. L'une et l'autre ont donc été deux rivières distinctes, qui n'ont conflué que sur différents aspects. Quant à l'ouvrage, je l'ai finalisé au moment où j'entamais la partie empirique de ce même projet DipLab. Cependant, ce dernier s'inscrit dans une certaine continuité, non sans marquer aussi une évolution par rapport à ce que j'ai pu écrire dans le livre. Alors que dans En attendant les robots, j’insiste sur les processus de délocalisation du travail du clic, les résultats de DipLab m'ont permis de montrer l'enracinement de ce même travail dans des contextes nationaux comme celui de la France. Tout se passe donc comme si, entre ce projet et l'ouvrage, s'était produite une pollinisation réciproque.

\section{《 Je travaille sur les technologies numériques et leur impact sur la société. Il s'agit là d'" objets à multiples entrées ", qui demandent à chaque fois des compétences qui sont autant issues des SHS que des sciences exactes i)}

- Qu'en est-il cependant du rôle de la MSH Paris-Saclay en ce qui concerne la diffusion et la communication autour de l'ouvrage?

Vous faites bien d'aborder cet autre aspect des activités de la MSH, car c'est bien une autre de ses missions que de contribuer à faire connaitre les travaux de ses chercheurs. En l'occurrence, mon ouvrage fera l'objet, le 6 décembre 2019, d'une journée d'études soutenue par la MSH, avec les auteurs de la revue L'Homme \& la Société et d'autres chercheurs discutants. Je suis par ailleurs invité à participer l'année prochaine à un Petit Déjeuner Durkheim, organisé par Florent Le Bot avec la MSH Paris-Saclay. Autant d'occasions d'échanges interdisciplinaires qui sont non seulement encouragés, mais encore facilités par la MSH. 


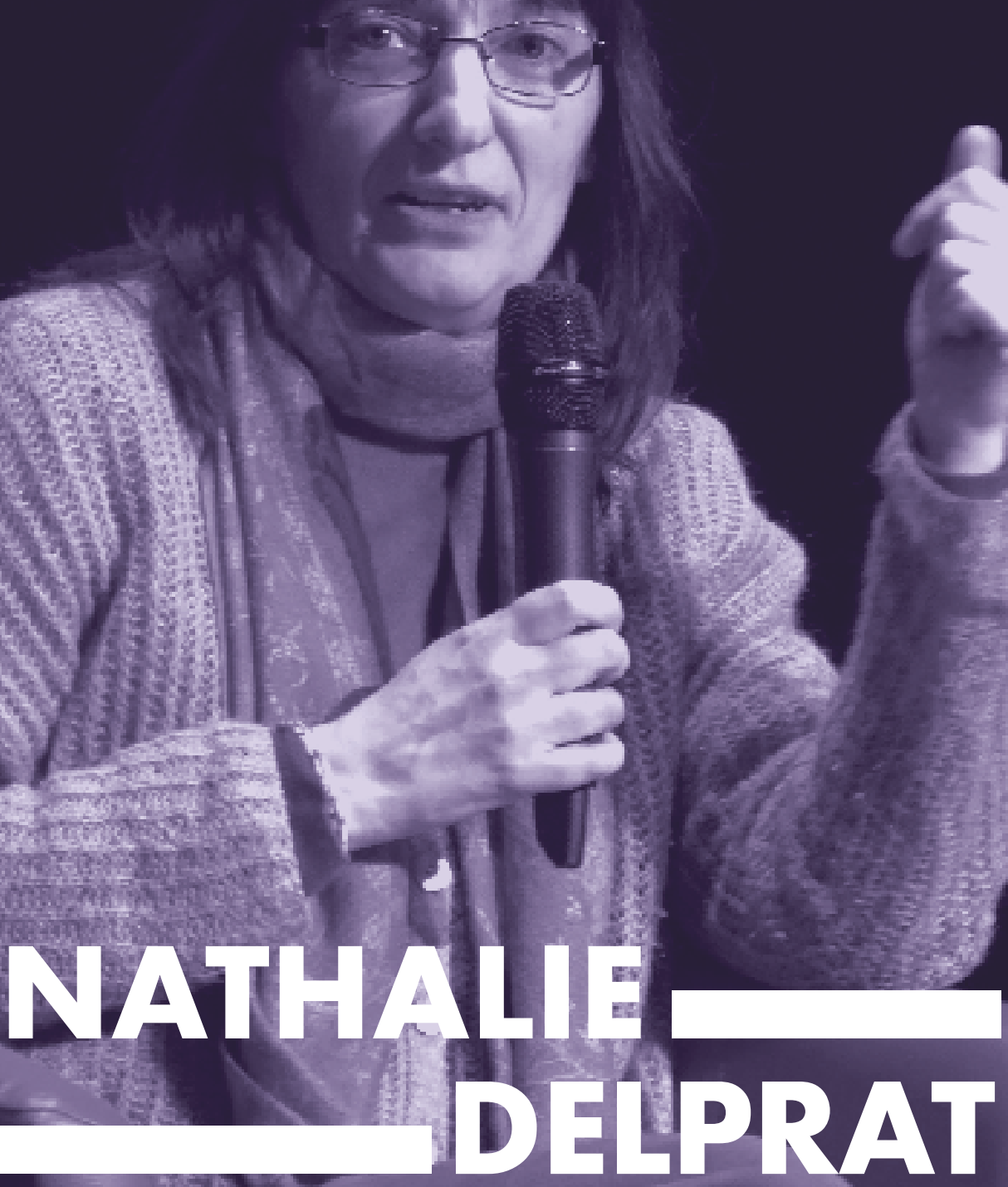

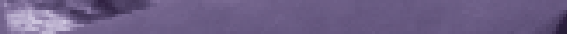

Quand l'art et la science inventent ensemble d'autres explorations de soi 


\section{Nathalie DELPRAT \\ Maître de conférences à Sorbonne Université Chercheure au Limsi (UPR 3251)}

- Comment en êtes-vous venue du traitement du signal appliqué à la mécanique des fluides à une perspective art-science?

En fait, c'est le chemin inverse que j'ai accompli. Je suis physicienne de formation, mais j'ai toujours été intéressée par l'approche art-science. En parallèle de mes études scientifiques, j'ai poursuivi des études musicales. Ensuite de 1988 à 1992, j’ai fait une thèse en informatique musicale avec Jean-Claude Risset [Médaille d'or du CNRS en 1999] à Marseille sur l'analyse et la synthèse des sons musicaux par ordinateur. J'ai suivi aussi des cours en musique et musicologie du $\mathrm{Xx}^{\mathrm{e}}$ siècle à l'Ircam (Institut de recherche et coordination acoustique/musique) et des master class avec des compositeurs comme Pierre Boulez ou Luigi Nono au centre Acanthes à Villeneuve-lès-Avignon.

\section{- Quelque chose de proprement passionnant!}

Oui, tout à fait. Pour en revenir à ma thèse, elle a donné lieu au développement d'une méthode originale en traitement du signal, que j’ai pu ensuite appliquer à des signaux en mécanique des fluides, au Laboratoire de modélisation en mécanique de l'Université Pierre et Marie Curie où j’ai été recrutée. En 2008, j’ai intégré le Limsi, et son groupe de recherche en audio et acoustique.

À partir de là, j’ai développé un axe de recherche art-science dans le cadre de la thématique transversale VIDA (Virtualité Interaction 
Design et Art), dont je suis la co-responsable depuis 2012. Au fil des années, cet axe est devenu l'essentiel de mon activité de recherche avec un élargissement important vers les sciences humaines et le développement de projets en art-science-philosophie, à l'image des projets Biomorphisme ou ELEMENTA.

- Comment le développement de cet axe de recherche a-t-il été reçu dans le monde des chercheurs ingénieurs avec lesquels vous étiez amenée à interagir? Partageaient-ils la même appétence que vous pour ce dialogue art-science?

(Sourire) Il faut garder à l'esprit que, dans les années 2000, il était difficile pour un scientifique de valoriser et même de faire de la recherche en artscience, a fortiori dans un laboratoire qui n'était pas dédié initialement à cela. Les mentalités ont changé récemment, surtout depuis que l'Europe a décidé, en 2015, de soutenir des projets en science, technologie et arts dans le cadre de son initiative STARTS. Cette évolution m'a encouragée à assumer davantage une activité que je poursuivais de manière plus ou moins «souterraine » dans mon premier laboratoire. Au Limsi, la thématique art-science a fini par être reconnue en tant que thématique transverse du laboratoire à partir de 2012. Cette avancée doit beaucoup à Christian Jacquemin qui a été à l'initiative de VIDA. Nous avons, entre autres, participé à la création et au développement de La Diagonale ParisSaclay, la structure en charge du dialogue science et société, au sein de la nouvelle Université Paris-Saclay. Quant au Limsi, il a su capitaliser une expertise importante et reconnue à l'étranger. Jeter des passerelles entre art et science n'aura pas toujours été simple, mais nous y sommes parvenus !

- D'autant que le dialogue art-science que vous promouviez cherchait à s'inscrire dans une logique de cocréation et de recherche partagée et non à faire de l'art un simple supplément d'âme...

Parfaitement, et c'est important de le souligner. Encore aujourd'hui, la vision du dialogue art-science que je défends n'est pas communément partagée. Il s'agit de faire reconnaître la création artistique comme une composante de la démarche de recherche. Pour l'heure, le paradigme dominant consiste à rapprocher un artiste et un chercheur, le temps 
d'un projet, pour la création d'une œuvre ou de la médiation scientifique dans une démarche croisée. Une vision que je ne récuse pas. Mais force est de constater qu'il est encore difficile de convaincre de l'intérêt d'une recherche scientifique avec et par l'art.

- Revenons-en à votre projet ELEMENTA. Si vous deviez le «pitcher »...? ELEMENTA, qui a impliqué trois laboratoires CNRS et un pôle national supérieur culturel, se propose d'étudier l'impact sensoriel et émotionnel d'une transformation virtuelle du corps en une matière élémentaire. L'expérience permet à la personne d'expérimenter son corps dans une autre matérialité à travers l'interaction avec des avatars ayant leurs propres qualités physiques.

Ce projet s'appuie sur les résultats obtenus avec le dispositif interactif RêvA développé au Limsi avec des avatars-nuages. Le dispositif se compose d'une caméra Kinect, qui filme la personne et extrait son squelette numérique. Chaque point du squelette est un émetteur de particules dont le paramétrage va permettre de fabriquer différents rendus de nuages - des plus compacts au plus évanescents, des plus proches de la silhouette humaine aux plus abstraits comme l'avatar-cirrus où seul le haut du corps est simulé par des lignes horizontales.

L'avatar est vidéoprojeté sur un grand écran et il n'est pas nécessaire de porter un casque ou des lunettes pour interagir en temps réel avec lui. On peut ajouter un effet de vent contrôlé par les mains, de façon à déplacer le corps virtuel dans l'espace, ce qui peut éventuellement provoquer sa dissolution. L'ensemble s'appuie sur un nouveau paradigme de recherche, que j’ai appelé «expérience de Rêverie augmentée ».
《 (...) la vision du dialogue art-science que je défends n'est pas communément partagée. Il s'agit de faire reconnaître la création artistique comme une composante de la démarche de recherche \} 
- «Rêverie augmentée »?

L'expression fait référence aux travaux du philosophe Gaston Bachelard sur l'imagination matérielle, mais aussi à l'augmentation par l'imaginaire de l'expérience sensori-motrice par laquelle le sujet appréhende son corps. Cette approche phénoménologique du lien à l'image, rendue possible par le rôle de l'imaginaire dans la boucle d'action-perception, s'inscrit dans un questionnement plus général sur la place de l'outil numérique dans l'étude de la construction du sujet. Ainsi, ELEMENTA permet d'étudier l'impact de l'effacement virtuel des frontières du corps sur le sentiment de soi, le ressenti émotionnel.

- Comment réagissent les personnes qui se prêtent à l'expérience?

La majorité des personnes la jugent plutôt agréable - certaines font état d'illusions perceptives particulières comme la sensation de matière à l'intérieur de leur corps. En revanche lors des entretiens réalisés à l'issue de l'expérience, quelques personnes rapportent un sentiment d'angoisse avec l'impression que "leur corps se vide », "se morcelle, » ou que "leur moi part en lambeaux ». C'est pourquoi l'objectif est de mieux comprendre l'influence de l'intéroception, autrement dit le ressenti de l'état interne du corps, et aussi le rôle de l'imaginaire, sur les mécanismes de perception du corps et le sentiment de soi.

- Quitte à vous ouvrir à d'autres disciplines?

Oui. ELEMENTA nous a déjà amenés à nous tourner vers différentes théories des neurosciences comme celle sur le self-embodiment par exemple, qui ancre la représentation du moi dans la perception des états internes du corps ou les « sentiments d'arrière-plan » dont parle le médecin et neurologue Antonio Damasio pour désigner des strates émotives à un niveau de conscience minimal, mais également de la psychanalyse - la notion de moi-peau, telle que développée par le psychanalyste Didier Anzieu sur les liens entre enveloppes psychiques et limites du corps.

- Avec quelles applications possibles? 
En matière de médiation thérapeutique, ELEMENTA pourrait permettre de proposer une alternative originale aux pratiques couramment utilisées en milieu psychiatrique, en utilisant l'interaction " matérielle » avec des représentations non figuratives du corps. Cela pourrait aider dans la revalorisation de l'image de soi, pour la régulation des émotions ou la modulation de la perception de douleurs globales. Le retour des personnes ayant eu un vécu positif de l'expérience fait état de sensations de flotter, de voler ou d'être plus léger avec les avatars-nuages, mais aussi de sentiments plus étranges, difficiles à décrire. Il faut donc comprendre ce qui se passe au niveau de la conscience corporelle.

Nous sommes actuellement en train de monter une étude pilote avec Catherine Jousselme, professeure à l'Université Paris-Saclay et pédopsychiatre à la fondation Vallée pour adapter le protocole à différentes pathologies, mais aussi pour mener une étude plus approfondie sur les ressentis liés au sentiment de déstabilisation de soi ou de perte du corps. L'objectif est de voir dans quelle mesure l'impossibilité de réajustement des frontières corps-moi dépend du profil psychologique du sujet, d'éventuels traumatismes ou pathologies.

- Quelles sont les autres retombées possibles?

Dansle domaine informatique, ELEMENTA aura permisle développement de nouveaux rendus de matière pour générer différents types d'avatars : en plus des avatars-nuages que j'évoquais, il y a maintenant des avatarsflammes, des avatar-bulles ou pluie. Nous terminons aussi la mise au point d'un nouveau mode de contrôle de la matière par le souffle et la voix. Pour cela, un séquenceur interactif a été implanté, ce qui permet d'envisager une plus grande liberté pour adapter le programme à de nouveaux protocoles expérimentaux avec des patients ou des chanteurs.

\section{- Et sur le plan artistique?}

Sur ce plan-là, le partenariat avec le projet Biomorphisme porté par le Centre Gilles Gaston Granger (CGGG) m'a permis d'exposer plusieurs œuvres, dont deux créées à partir d'une nouvelle version d'ELEMENTA réalisée avec la collaboration de Nicolas Ladevèze (Limsi, responsable 
informatique du projet) et les participations de Yujiro Okuya (Limsi), Jean-Michel Couturier (BlueYeti) et Patrick Sanchez (LMA). Le travail a été exposé à la Friche la Belle de Mai à Marseille, de novembre 2018 à janvier 2019, avec notamment l'installation interactive « Murmure » qui permet aux spectateurs d'engager un dialogue avec des doubles matériels éphémères, accompagnés par des bruits de vent, le souffle d'une flûte imaginaire ou les échos de gouttes d'eau. Une performance dansée a été également proposée à partir du travail réalisé avec des danseurs du Pôle national supérieur de danse et du Ballet national de Marseille. ELEMENTA m'a inspiré d'autres projets d'œuvres que je compte réaliser dans un futur proche, à l'occasion d'autres événements.

Autant le dire, cette approche est tout sauf simple. Elle a demandé beaucoup de temps et de travail, mais m'a confortée dans l'idée de poursuivre cette exploration et, surtout, de renforcer une des pistes les plus prometteuses de cette recherche, à savoir le développement d'une dynamique art-science-philosophie.

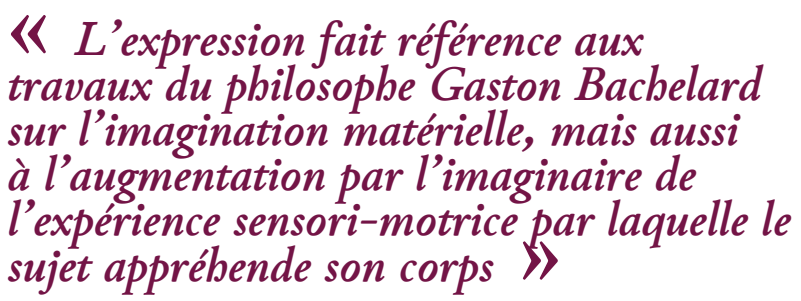

- Quelle a été la réception réservée à vos travaux?

Un documentaire va être réalisé à l'horizon 2020 par le CNRS, suite au prix CNRS Image que j’ai reçu en juin 2018 dans le cadre du festival Sciences en Lumière 2018. J'ai obtenu, en outre, une délégation CNRS de six mois au sein du CGGG, un laboratoire d'épistémologie et de philosophie des sciences, notamment pour formaliser la théorie sous-jacente à mon approche, à la lumière de la poétique bachelardienne des éléments qui permet la prise en compte de la dimension imaginative et créatrice du sujet dans la construction du sentiment de soi. Enfin, j’ai obtenu une résidence courte en mai 2020, à la Villa Médicis, pour démarrer la rédaction d'un essai sur les aspects poétiques et artistiques du paradigme de Rêverie augmentée. 

Professeur en sciences de gestion à la Faculté Jean Monnet de l'Université Paris-Sud, il est par ailleurs, depuis 2013, le Rédacteur en chef de la Revue française de gestion. Début 2019, il a été lauréat, avec Damien Mourey (IDHES, ENS ParisSaclay, CNRS) d'un appel à séminaire pour le projet " Academic All-Star Game ", un cycle de neuf conférences destiné à faire connaître la recherche en sciences de gestion et à susciter des vocations parmi les étudiants. La première édition avait pour thème l'avenir de la recherche en stratégie et management (" Recherche en stratégie et management : mort clinique ou renaissance ? "). À chaque conférence, un duo de chercheurs francophones de renommée internationale était convié à partager leurs sentiments et visions sur une thématique particulière.

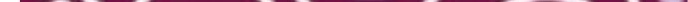




\section{Jean-Philippe DENIS \\ Professeur des universités à l'Université Paris-Sud Chercheur au Ritm (EA 7360, PSud)}

- Si vous deviez pour commencer par rappeler le statut des sciences de gestion au sein des $S H S . .$.

Comparée à la plupart des SHS, la gestion est une discipline relativement jeune. En France, l'émergence d'une recherche académique dans ce domaine remonte à une cinquantaine d'années. Sa structuration institutionnelle est intervenue avec la création du réseau des IAE (Instituts des administrations des entreprises), puis de l'Université de Paris-Dauphine, en 1968, et, à la fin des années 1970, avec l'organisation du premier concours d'agrégation du Supérieur en gestion. Jusqu'alors, la gestion était considérée comme une discipline professionnelle.

Cela étant dit, l'idée suivant laquelle elle est une discipline jeune n'est pas admise par tous les spécialistes du domaine. Certains - je pense notamment à l'équipe du Centre de gestion scientifique (CGS) de Mines ParisTech - considèrent que c'est au contraire une discipline très ancienne.

Un autre débat interne n'a cessé d'agiter la discipline, autour de sa capacité à exister par elle-même. Dans la plupart des ouvrages de référence, on retrouve l'idée d'une discipline qui ne se serait constituée qu'à partir d'emprunts à d'autres disciplines, l'économie et la sociologie, principalement. La gestion leur aurait emprunté la plupart de ses concepts, voire de ses fondements théoriques. Cela en fait tout à la fois l'intérêt et le drame : la gestion en est souvent réduite à être assimilée à une forme d'économie ou de sociologie appliquée à l'entreprise, sans identité propre. 
- S'agit-il de renier ces emprunts?

Non, bien sûr - ces emprunts font partie de son histoire. Il s'agit plutôt de permettre à la discipline de faire valoir les concepts et théories qu'elle a été et est encore en mesure de produire par elle-même, en s'intéressant à bien plus qu'à l'entreprise, à laquelle on a l'habitude de la réduire. Les sciences de gestion travaillent depuis l'origine sur l'action collective et devraient, donc, entretenir avec la sociologie, l'économie, mais aussi les sciences politiques et le droit un dialogue plus fécond. Elles traitent aussi des problématiques de gouvernance et de gouvernement, de stratégie, dans le domaine aussi bien civil que militaire. En disant cela, je ne veux pas dire qu'il faille relativiser l'intérêt de l'entreprise comme objet d'étude. Au contraire, il y a urgence pour les gestionnaires et les autres disciplines de mieux appréhender le monde de l'entreprise, aujourd'hui plus que jamais à l'heure où certaines d'entre elles semblent être plus puissantes que les États. Non sans que cela questionne d'ailleurs les modes de gestion, qui manifestent ainsi l'impact direct et indirect qu'ils peuvent avoir sur nos sociétés.

- Quel peut être l'apport de l'inscription des sciences de gestion dans le champ d'une MSH ?

Cette inscription est essentielle. Elle leur permet de poursuivre un dialogue avec l'ensemble des disciplines, dans une position d'égal à égal. Autant le dire : ce dialogue ne va pas de soi. À chaque discipline, ses traditions, ses concepts, théories et méthodes, ses objets, sinon ses manières de les appréhender. Cela étant dit, le principe même d'une conversation est de mettre au jour des désaccords sans rompre pour autant le dialogue. Comme carrefour des disciplines, la MSH me paraît le lieu le plus approprié pour amorcer ou poursuivre les débats, à travers des projets à vocation réellement interdisciplinaire.

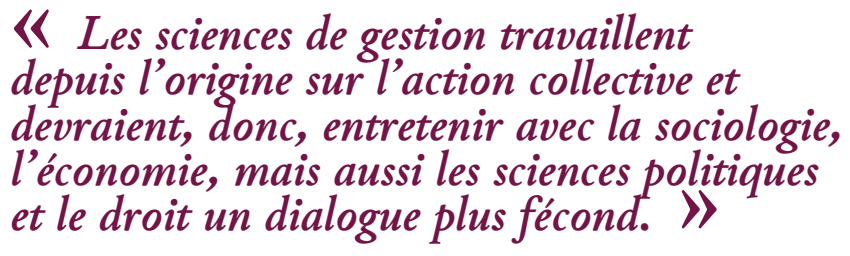


- Venons-en à une contribution concrète de votre part : l'Academic All-Star Game. Pouvez-vous en rappeler la genèse et les principes?

L'Academic All-Star Game est un cycle de conférences organisé avec mon collègue Damien Mourey (IDHES, ENS Paris-Saclay, CNRS) en partenariat avec l'Université Paris-Sud et la faculté Jean Monnet, ainsi que le RITM (le laboratoire de recherche en économie et en gestion de l'Université Paris-Sud). À chaque conférence, un duo de collègues, différents de par leur ancrage disciplinaire, leur thématique de recherche et/ou leur génération, mais partageant les mêmes convictions : l'intérêt d'une recherche en gestion, la nécessité d'en régénérer les débats.

À rebours de la logique de structuration de l'Université Paris-Saclay, nous avons pris le parti de n'inviter que des professeurs de gestion extérieurs à cette université : d'universités ou de grandes écoles, de Paris ou de province. Certes, nous y avons convié Ève Chiapello, qui préside le Conseil scientifique de la MSH, mais elle intervenait à un autre titre, celui de sociologue de l'EHESS.

Concernant la composition du binôme, nous nous sommes demandé s'il fallait, dans une perspective d'interdisciplinarité, croiser les regards d'un « gestionnaire » et d'un enseignant-chercheur d'une autre discipline. Finalement, nous avons pris un autre parti, celui de ne convier que des spécialistes de gestion, de façon à donner à voir à quel point leurs parcours et ancrages disciplinaires sont variés, certains étant devenus sociologues, à l'image d'Ève Chiapello, d'autres ayant un profil d'économiste ou même de philosophe (de sorte qu'une conférence a pu être l'occasion de traiter de philosophie pragmatique).

- La première édition avait pour intitulé générique "Recherche en stratégie et management: mort clinique ou renaissance?", avec plusieurs séances « au chevet de la gestion ou du management »... La situation est-elle aussi dramatique?

Les sciences de gestion sont, de fait, à un moment clé de leur histoire : à force d'avoir voulu se scientificiser, elles sont sur le point de perdre leur identité. Force est, en outre, de constater une méconnaissance dans les institutions scientifiques, universitaires et, pour tout dire, dans la société, de la simple existence d'une recherche en sciences de gestion. J'y reviens. 
Des collègues d'autres disciplines en sont encore à s'étonner que puisse exister une recherche dans ce domaine... Quand un professeur de gestion s'exprime dans les médias, il est souvent présenté comme sociologue ou économiste... Il est rare de voir des professeurs de gestion sollicités en tant que tels. Le débat reste d'ailleurs récurrent quant à savoir si gestion est le bon terme, s'il faudrait parler de « gestionologue »... D'aucuns proposent « orgologues » ou « orgologie », pour désigner la science des organisations... Ces débats témoignent de la difficulté à faire reconnaitre les sciences de gestion comme une discipline à part entière.

\section{《 (...) en gestion, la pédagogie et la recherche sont en réalité inextricablement liés 》}

- Comment votre cycle de conférence s'est-il employé à contribuer à leur renaissance?

Il nous a semblé que la meilleure façon de faire était d'en confier l'organisation et l'animation aux étudiants eux-mêmes, avec l'espoir de susciter chez eux l'envie de s'investir dans la recherche. C'est à eux que revenait le soin de contacter les intervenants, de préparer leur venue, en se plongeant préalablement dans leurs écrits. L'appellation même d'Academic All-Star Game est de leur initiative.

- Au-delà des débats internes ou avec les autres SHS, l'enjeu de ce cycle n'était-il pas aussi de montrer que les sciences de gestion pouvaient aussi contribuer à éclairer l'actualité?

Si. À cet égard, le fait d'avoir traité du mouvement des Gilets jaunes n'était pas anodin. Il témoigne du fait que les sciences de gestion ont aussi des choses à dire sur les enjeux de société qui font l'actualité. De même, la session consacrée à l'entreprise comme support à la création collective, avec Armand Hatchuel, a été au cœur de l'actualité, puisqu'elle a permis de revenir sur la loi Pacte, qui reconnait, entre autres choses, le principe d'« entreprises à mission ». 
- Au final, en quoi votre projet réunissait-il les critères requis pour être retenu dans le cadre de l'appel à projet Séminaires?

Cela a été justement l'objet d'intenses débats car, reconnaissons-le, de prime abord, la MSH aurait pu invoquer plusieurs arguments pour renoncer à soutenir notre projet, à commencer par le fait que nous ne sollicitions que des professeurs de gestion, de surcroît extérieurs à l'Université de Paris-Saclay. Sans compter ce portage par les étudiants, qui pouvait le faire apparaitre comme un projet pédagogique et non de recherche. Je sais d'autant plus gré à la MSH d'avoir entendu nos arguments.

\section{- Quels étaient-ils?}

Par la qualité des intervenants, l'Academic All-Star Game est, de fait, aussi un programme de recherche, étant entendu qu'en gestion, la pédagogie et la recherche sont en réalité inextricablement liés. Il aurait donc été réducteur de s'en tenir à de la recherche.... J'irai plus loin en considérant que la gestion est un art pratique, en plus d'être une science.

Si les intervenants ne sont pas de l'Université Paris-Saclay, en revanche, les étudiants ayant participé à l'organisation et l'animation du projet le sont tous : ils sont en licence et en M2 en Management stratégique porté par trois établissements de l'Université Paris-Saclay (l'Université ParisSud, l'ENS Paris-Saclay et l'UVSQ).

\section{- Quels ont été les effets produits par cette première saison?}

Vous dites "saison ", ce qui suggère qu'il y en aura une suivante. La question reste d'actualité. Organiser et animer un tel cycle de neuf conférences de trois heures chacune, cela prend du temps!

Cela étant dit, la première saison n'en a pas moins déjà des prolongements. Les conférences ont été systématiquement filmées et complétées d'interviews (les vidéos sont disponibles sur le site de la $\mathrm{MSH}$ via YouTube). Chaque séance a également fait l'objet d'une valorisation dans The Conversation sous la forme d'une chronique rédigée par les étudiants. Enfin, un numéro spécial de la Revue française de gestion, coordonné par Alain-Charles Martinet et Pierre-Michel Menger, paraîtra à la fin de l'année, avec des contributions des intervenants du cycle. 


\section{Jean-Philippe Denis}

Comme nous l'espérons, le premier cycle aura été un vecteur de rayonnement pour les sciences de gestion, mais aussi la MSH et l'Université Paris-Saclay, que nos intervenants extérieurs auront pour beaucoup découvertes à cette occasion. Quant à savoir s'il a suscité des vocations parmi les étudiants, il est encore trop tôt pour le dire. 


\section{ANAIS

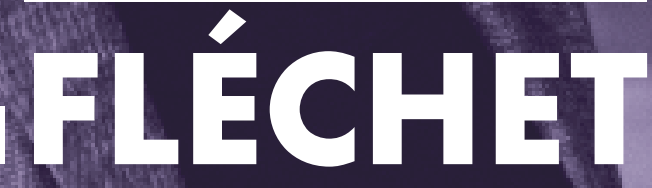 \\ Relations internationales et globalisation culturelle au rythme de la musique}




\section{Anaïs FLÉCHET \\ Maître de conférences à l'UVSQ \\ Directrice adjointe du CHCSC}

- Comment en êtes-vous venue à vous intéresser aux échanges culturels et de la musique en particulier au prisme des relations transatlantiques?

Pour des raisons familiales, l'Amérique latine est un continent qui m'est cher. Je connais bien en particulier le Brésil. Lorsque j’ai commencé mes études en histoire culturelle, je me suis naturellement intéressée de près à la pratique musicale latino-américaine, par ailleurs riche et intéressante en soi. Mes mémoires de master et de thèse ont porté sur les circulations musicales entre l'Amérique latine et l'Europe en général, le Brésil et la France en particulier. J'y analysais les phénomènes de mode, de métissage, de circulation des sons et des ouvres artistiques, et ce dans le contexte plus général de ce qu'il est convenu d'appeler la «globalisation culturelle », en me focalisant sur l'espace atlantique, autrement dit les échanges entre les deux Amériques, l'Afrique et l'Europe.

Depuis, $j$ 'en suis venue à porter mon attention sur les usages politiques et plus précisément diplomatiques de la musique, à la manière dont les circulations musicales pouvaient éclairer, au-delà des enjeux esthétiques et des logiques du marché de la culture, les relations politiques entre les États, que ce soit au travers de la propagande ou, dans le cas qui m'intéressait à l'époque, de l'exil politique - j'ai beaucoup travaillé sur les figures de musiciens exilés. 
- Dans quelle mesure vos projets labellisés par la MSH vous ont-ils permis d'approfondir ou d'ouvrir de nouvelles perspectives de recherche?

La première aide dont j'ai bénéficié a été allouée en 2015 conjointement par la MSH et le département SHS de Paris-Saclay. D'un montant de 1500 euros, elle m'a permis d'organiser une journée d'études sur le thème "Musique et nation dans l'entre-deux-guerres Europe-Amériques », avec Martin Guerpin et Philippe Gumplowicz, qui enseignent la musicologie à l'Université d'Évry Val-d'Essonne. Cette journée eut un rôle décisif dans la suite de mes recherches et ce, à un double titre. D'abord, elle m'a permis de rencontrer des collègues musicologues et d'instaurer avec eux un dialogue on ne peut plus fécond et durable. Non que les musicologues et les historiens qui, comme moi, travaillent sur l'histoire politique, culturelle et sociale de la musique n'aient pas déjà l'occasion de dialoguer, mais c'est le plus souvent de manière ponctuelle, voire informelle.

Ensuite, cette même journée devait avoir des prolongements dans des projets communs. Elle nous a, pour commencer, convaincus de travailler ensemble dans une perspective à la fois historique et musicologique, en explorant une histoire de la musique qui soit aussi une histoire par la musique et pas seulement une histoire où la musique en resterait à l'arrièreplan d'événements politiques ou de phénomènes sociaux, comme c'est souvent le cas. En sens inverse, l'enjeu était aussi que les musicologues accordent eux-mêmes une plus grande importance à des questions dont traite l'histoire culturelle et sociale, à savoir les pratiques et représentations, les enjeux politiques, les marchés culturels... Concrètement, c'est à la suite de cette journée d'études que Martin Guerpin, Philippe Gumplowicz et moi avons entrepris, avec le concours de Barbara Kelly, professeure de musicologie et directrice de recherche au Royal Northern College of Music, de monter un réseau européen, devenu depuis international, de recherche sur les rapports entre musique et histoire culturelle, politique et sociale.

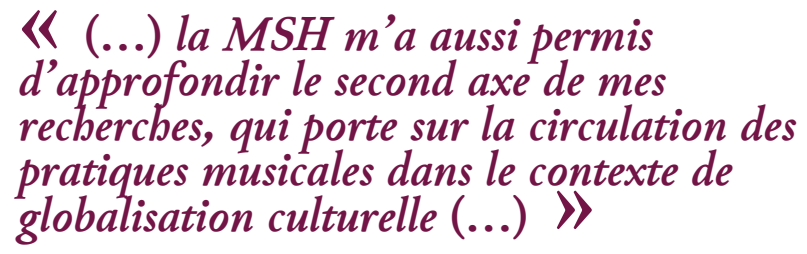


- Quel a été l'apport de l'appel à projets Émergence sur le thème "Musique et sorties de guerres »?

Il a permis de conforter la dynamique initiée par la journée d'études, en portant l'attention sur les processus de démobilisation culturelle, les musiques de deuil et de commémorations, les reconfigurations esthétiques et institutionnelles, ainsi que sur les liens entre musique et diplomatie. Avec mes collègues cités plus haut, nous avons monté un colloque sur ce thème, qui eut lieu en octobre 2018, à l'Université de Montréal. Le succès fut au rendez-vous, à en juger par le nombre de participants et la qualité des contributions et des échanges. Les actes paraitront en 2020, en anglais.

Mais la MSH m'a aussi permis d'approfondir le second axe de mes recherches, qui porte sur la circulation des pratiques musicales dans le contexte de globalisation culturelle, à travers d'autres appels à projets dont j'ai été lauréate.

Le premier, monté avec des collègues de São Paolo et d'Amérique du Nord (avec lesquels j’avais gardé contact suite à mon année passée à l'université de Berkeley, en Californie), s'intitule "Transatlantic Cultures ». Il vise à constituer un dictionnaire d'histoire culturelle en ligne, sur les circulations culturelles dans l'espace atlantique, de la fin du $\mathrm{XVIII}^{\mathrm{e}}$ siècle (autrement dit, à partir des révolutions américaines), jusqu’à nos jours. Édité en quatre langues, dans le cadre d'un projet déjà financé par l'ANR, il réunit à ce jour une quarantaine de chercheurs spécialistes des États-Unis, de l'Europe, de l'Amérique latine et de l'Afrique : aussi bien des historiens que des spécialistes de littérature, de théâtre, des sociologues, des anthropologues... C'est dire s'il est ambitieux et n'aurait pu voir le jour sans le soutien de l'ANR, du fonds France-Berkeley et de l'Agence pour la recherche au Brésil.

Ce même projet a cependant manqué de peu d'être compromis par la crise qui affecte depuis maintenant quelques années ce pays : les subventions brésiliennes ont été drastiquement réduites, tant et si bien que nous nous sommes retrouvés dans une impasse. 
- C'est là qu'est intervenue la MSH ?

Parfaitement. Le dictionnaire étant conçu sur la base d'une plateforme numérique, j'ai proposé à la MSH d'en développer le volet « humanités numériques » de façon à valoriser les travaux déjà engagés dans le cadre de Transatlantic Cultures. Cette proposition a été soutenue par un appel à projets Maturation, dont j'ai été lauréate en 2018. Concrètement, ce soutien nous a permis de réaliser le web design de la plateforme, mais aussi de travailler à l'interface utilisateur, qui contribue grandement à la réussite $\mathrm{du}$ projet, non sans innover au plan technique, avec la conception de nouveaux outils de visualisation. Une autre forme d'interdisciplinarité s'il en est, entre SHS et informatique. Par ailleurs, je travaille actuellement avec un postdoctorant en informatique, sur des formes de cartographies dynamiques pour visualiser la circulation de flux culturels, liés aux tournées d'artistes, aux importations de livres, etc.

Naturellement, il y a un enjeu de diffusion auprès d'un large public et, donc, une attention à recourir à un langage accessible, tout en croisant les formes de médias.

\section{《Le premier intérêt de la MSH, c'est son accompagnement dans la durée, au fil d'appels à projets. Cela permet à des idées de gagner en maturité et en ampleur (...) I)}

- Et le projet "Atlantic argentique ", comment s'est-il inscrit dans cette dynamique de recherche?

Ce projet vise à montrer comment la technologie photographique s'est imposée comme un moyen de communication de premier plan dans des domaines aussi divers que la presse, l'éducation, la recherche ou encore la publicité. Il s'inscrit parfaitement dans la continuité du projet Transatlantic Cultures qui, comme vous l'avez compris, ne concerne pas que la musique, mais les circulations culturelles dans leur ensemble.

Précisons encore qu'il est porté par Clara Bouveresse, maitre de conférences et chercheure en histoire de la photographie et des études visuelles, au laboratoire Slam de l'Université d'Évry Val-d'Essonne. C'est 
en découvrant notre projet de dictionnaire et dans le dialogue avec des collègues de l'Université Paris 3 qu'elle nous a proposé d'élargir l'étude des circulations culturelles au domaine de la photographie. Je me suis associée à elle pour soumettre le projet à l'appel à projets Émergence 2019. Concrètement, il se traduira par un colloque au musée du Jeu de Paume, à Paris. Une perspective d'autant plus intéressante qu'il sera organisé en lien avec une exposition. Ce qui correspond à ce que j'ai toujours eu à cœur : combiner de la recherche universitaire, sur des sujets pointus, avec des manifestations culturelles et artistiques. La moindre des choses, me semble-t-il, dès lors qu'on traite d'histoire culturelle.

\section{- Au final, quelle est la valeur ajoutée de l'apport de la MSH ?}

Le premier intérêt de la MSH, c'est son accompagnement dans la durée, au fil d'appels à projets. Cela permet à des idées de gagner en maturité et en ampleur en bénéficiant, par un effet boule de neige, d'autres financements. Je lui en suis d'autant plus redevable que j'y ai vu une marque de confiance. En 2015, j'étais à mille lieues d'imaginer que la journée d'études donnerait lieu à d'autres projets, de surcroît plus ambitieux au regard de leur ouverture disciplinaire et à l'international.

Ce faisant, la MSH m'a permis de me rapprocher de collègues français et étrangers avec lesquels j'avais envie de collaborer, dans un esprit réellement interdisciplinaire. Des projets que nous ne pensions pas pouvoir réaliser aussi vite, voire tout court, ont pu voir le jour. À cet égard, le principe de l'appel à projets, même s'il ne doit pas se substituer aux dotations des laboratoires, qui demeurent notre soutien fondamental, a quelque chose de positif : c'est une invitation à couvrir de nouveaux chantiers. Ensuite, le fait que des appels soient régulièrement lancés, j'y reviens, permet d'entretenir la dynamique dans la durée. Ils suppléent les manques de moyens financiers dont pâtissent des universités, du fait des contraintes budgétaires, qui se traduisent pour certaines par des réductions drastiques des moyens alloués aux laboratoires. Une situation qui m'a incitée à me tourner vers l'extérieur pour trouver d'autres financements, en fonctionnant davantage dans une logique de recherche de financement, par projet. 
Au-delà de financements, la MSH nous donne accès aux outils et ressources du CNRS. Ce qui est précieux quand on sait que la majorité des laboratoires SHS de Paris-Saclay sont des Équipes d'accueil (EA). Il existe plusieurs UMR CNRS en SHS, mais elles sont moins nombreuses que dans le champ des sciences exactes. Certes, nous n'échappons pas à des démarches administratives, liées aux dossiers à remplir en réponse aux appels à projets. Mais une fois le projet retenu, la MSH nous assure un soutien logistique pour l'organisation de nos événements, mais également la diffusion des résultats de nos travaux. Au-delà de son aspect institutionnel, la MSH, c'est d'abord une équipe dévouée, qui, en plus d'être réactive, veille à rendre les choses toujours aussi fluides que possible, avec bienveillance et efficacité. 


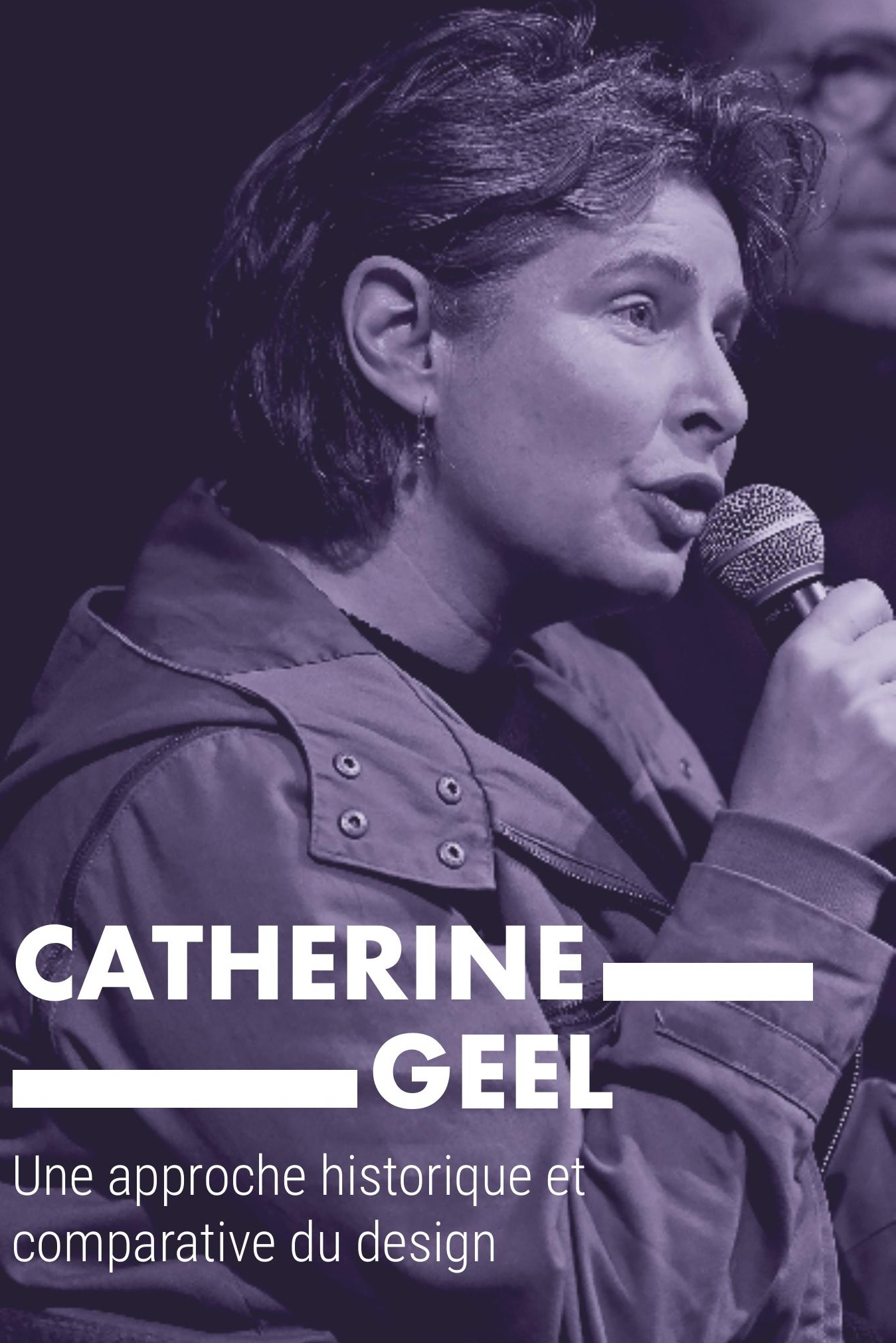


Professeur titulaire des écoles nationales supérieures d'art, enseignante à I'ENS Paris-Saclay et Sciences Po Paris, Catherine Geel promeut depuis plusieurs années une approche à la fois historiographique et comparative du design. Elle est par ailleurs éditrice de la revue de recherche franco-suisse Raddar.

Elle a été lauréate d'appels à projet MSH Paris-Saclay pour l'organisation de plusieurs manifestations : un séminaire " Histoire du design " (créé en 2018 et reconduit en 2019), une journée d'études «Photographie et Design. Deux arts industriels » (novembre 2018), enfin, une journée d'études organisée à Milan dans le cadre de la Triennale du design (appel à workshops 2019). Elle a également été lauréate en 2018 d'un appel à projets Maturation pour le projet Problemata 2.0, qui vise à la constitution d'une plateforme numérique de diffusion des travaux de recherche en histoire du design et en études critiques.

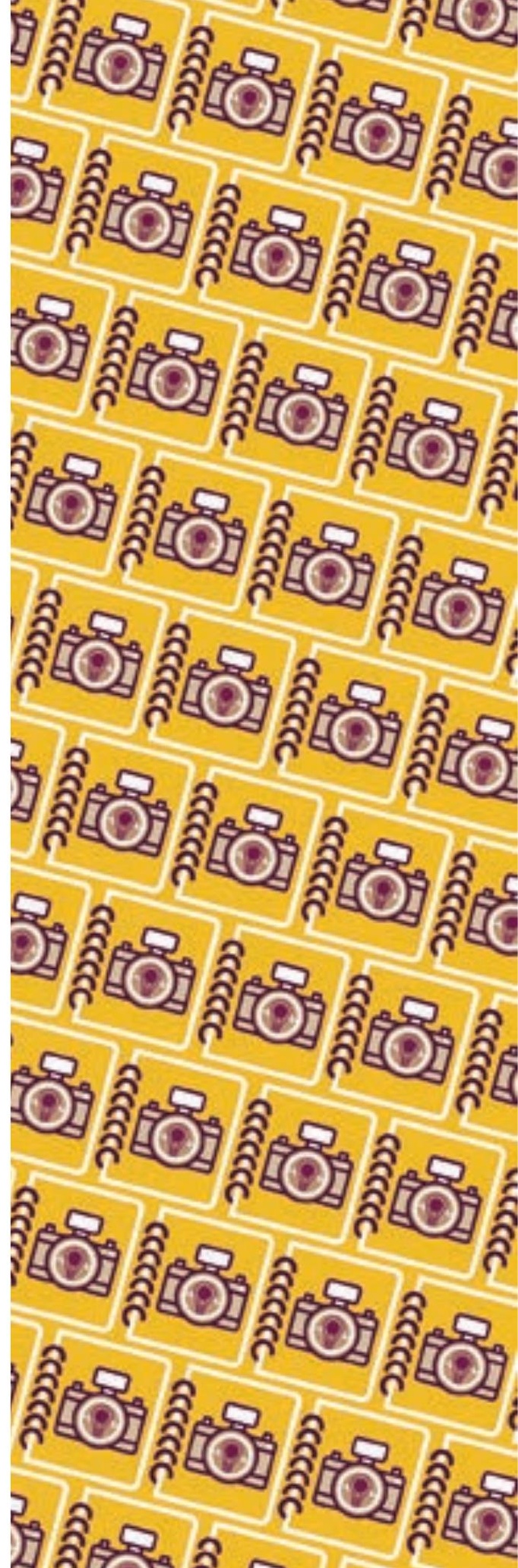




\section{Catherine GEEL \\ Professeur titulaire des écoles nationales supérieures d'art Enseignante à l'ENS Paris-Saclay et Sciences Po Paris}

- Comment en êtes-vous venue à cette approche à la fois bistorique et comparative du design?

Cet intérêt pour la mise en perspective historique du design est parti d'un constat : la France, comparée à d'autres pays, n'a pas développé d'histoire du design. Non que le design y soit apparu tardivement, mais sa pratique n'a pas été l'objet d'un travail historiographique aussi important que dans d'autres pays.

Si la France n'a pas de grande tradition historique en design, elle a, en revanche, une modernité dans ce domaine - qui réussit à faire passer Le Corbusier pour Français ! - et des pionniers - que l'on n'appelait pas encore designers - qui se sont battus au non d'une modernisation industrielle et sociale du pays. Une tradition que le père anglo-germanique de l'histoire du design, Nikolaus Pevsner a, quelque peu, gommée. À l'arrivée, force est de constater que le champ des études critiques et historiques est extrêmement développé dans les pays anglo-saxons et en Italie, où le design appartient à la fois aux secteurs universitaires, culturels et économiques. Je souligne économique, car c'est loin d'être le cas en France où cette inscription du design dans différents champs n'est ni naturelle ni installée. Or, si on considère la question écologique, par exemple, tout le corpus textuel, historique et théorique de la discipline dresse des perspectives et aborde les problématiques environnementales dès le XIX ${ }^{\mathrm{e}}$ siècle. Il est donc essentiel de développer des repères historiques propres au design, dans une perspective de compréhension des enjeux contemporains et à venir. 
- Ce à quoi vous vous êtes employée à travers de l'enseignement, de la recherche, le montage d'expositions...

C'est vrai que mon parcours témoigne d'un engagement multiple. En plus de mes enseignements comme professeure titulaire des écoles nationales supérieures d'art, j'enseigne à l'ENS Paris-Saclay et Sciences Po Paris ; j'ai par ailleurs cofondé et dirigé un master au Sandberg Instituut (Gerrit Rietveld Academie) à Amsterdam, et rejoint comme membre associée le Centre de recherche en design de l'ENS Paris-Saclay et l'Ensci-Les Ateliers. J'ai aussi une activité de commissaire d'exposition - j’ai été la commissaire de la représentation française à la Triennale de design pour son retour à Milan cette année, en 2019. J'ai été aussi productrice à France Culture (2005-2012) et, comme commissaire, cofondatrice du festival Design Parade. Bref, j’ai à cœur que les choses avancent disciplinairement. Cela étant dit, je ne suis pas seule à promouvoir une histoire du design. Nous sommes quelques-uns à installer un axe fortement historique, que d'autres diraient épistémologique : Emanuele Quinz à Paris 8 et l'EnsadLab, Annalisa Viati à Versailles, Antonella Tufano à la Villette. Sans compter plusieurs de mes anciens étudiants : Anthony Masure, directeur de la recherche à la Head (Genève) et Tony Côme, à l'EnsciLes Ateliers, qui développent des perspectives généalogiques ; ou encore David Bihannic, designer en data visualisation et maître de conférence à Paris 1, avec qui je codirige un ouvrage où 35 chercheurs français en design ré-analysent le Bauhaus à l'aune de travaux historiographiques contemporains. Ensemble, nous stimulons, nous travaillons les archives. Nous considérons qu'il n'y a pas de projet sans sa culture, pas d'assise disciplinaire sans une réflexion épistémologique. Et nous considérons que l'approche comparatiste est tout aussi essentielle. Pendant deux ans, nous avons ainsi invité une vingtaine de chercheurs étrangers au sein du séminaire de recherche mené avec Claire Brunet, Emanuele Quinz et Annalisa Viati. Une initiative, qui n'aurait pu se concrétiser sans le concours de la MSH Paris-Saclay.

Par ailleurs, nous avons été invités par les laboratoires Slam et CHCSC de l'Université d'Évry Val d'Essonne et de l'Université Versailles SaintQuentin-en-Yvelines à participer à un projet sur l'américanisation par 
les arts, qui insère le design dans une réflexion historique française. Un honneur que de partager cette recherche avec nos collègues, quand on songe que notre propre programme de recherche n'a que deux ans d'existence.

- Plusieurs autres de vos projets ont été soutenus par la MSH Paris-Saclay... Oui, à commencer par un séminaire "Histoire du design », organisé en 2018 et reconduit en 2019, avec pour vocation de contextualiser les pratiques des designers européens dans la seconde moitié du $\mathrm{xx}^{\mathrm{e}}$ siècle dans les débats de société. Il s'agissait d'analyser les procédures d'interpénétration des domaines qui concernent la discipline, en nous interrogeant sur la notion de design radical (1965-1974) spécifique à l'Italie et ce, à l'aune de cinq sujets : l'influence anglaise des années 1950 ; le décollage économique italien et sa critique marxienne ; la vie culturelle ; la valeur de l'innovation ; enfin, le renouvellement de la réflexion pédagogique sur l'enseignement du projet.

Avec Éléonore Challine, historienne de la photographie et maittre de conférence à Paris 1 et à l'INHA, nous avons ensuite bénéficié du concours de la MSH pour l'organisation d'une journée d'études « Photographie et Design. Deux arts industriels », en novembre 2018, puis d'une autre (appel à workshops 2019), à Milan, dans le cadre de la Triennale du design. Enfin, jai été lauréate d'un appel à projets Maturation pour le projet Problemata 2.0. Deux de ses volets relèvent clairement des humanités numériques : d'une part, la stimulation de la recherche en histoire du design et en études critiques via la diffusion en ligne de corpus relativement conséquents constitué en trois ans; d'autre part, une réflexion en design sur l'ergonomie d'une plateforme numérique et la normativité des protocoles proposés en matière de design des plateformes scientifiques.

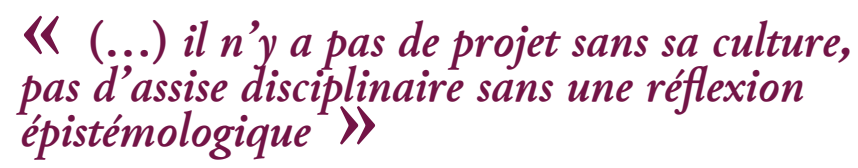


- Dans quelle mesure ces projets auront-ils permis d'ancrer l'bistoire du design dans le champ universitaire?

A priori, l'histoire du design n'est ni une histoire de l'art ni une histoire de l'architecture. Dans les universités étrangères du moins, elle s'est développée dans les départements de praticiens, avec de nombreux apports des sciences humaines. C'est dire si elle avait toute sa place à l'ENS ParisSaclay. Mais il est clair qu'elle gagnait à s'inscrire davantage dans le champ universitaire. C'est ce à quoi a contribué la MSH notamment à travers l'ambitieux projet Maturation que nous développons avec Huma-Num (TGIR du CNRS). Les discussions que nous avons avec les interlocuteurs de la MSH (en particulier son responsable de la coordination scientifique et son chargé de communication) ont permis de tisser des liens avec d'autres unités de recherche, non seulement plus pertinents, mais encore plus rapidement que nous l'aurions fait sans leur concours.

Rappelons que notre propre laboratoire est encore jeune. La MSH aura ainsi permis d'accélérer l'installation d'une histoire du design, par la mise à disposition de moyens, qui ont rendu possibles des échanges avec d'autres disciplines en France et avec des universités étrangères.

- Dans quelle mesure aura-t-elle contribué à la réception de vos travaux?

On aborde là un autre apport essentiel de la MSH, à savoir : le travail de diffusion et d'édition. De même que la journée d'études et le séminaire, le projet Maturation va nous permettre d'éditer ce qui a été fait ces toutes dernières années. C'est ce suivi et, j’ose le dire, la fidélité avec laquelle la MSH Paris-Saclay a labellisé nos séminaires et journées d'études, qui se sont matérialisés jusqu'en Italie, où nous avons été associés en mars dernier à la Triennale de Milan (l'équivalent pour le design de la Biennale de Venise en architecture), que j'évoquais. Avec le ministère de la Culture et l'Institut français, nous avons pu y présenter la recherche en design française au Palazzo. Nous y avons même eu la visite du ministre de la Culture en personne. 
- Dans quelle mesure les projets MSH vous ont-ils confortée dans la nécessité de l'interdisciplinarité, mais encore dans l'idée qu'elle était possible?

Le design est par essence une discipline interdisciplinaire. Ses praticiens ne peuvent faire autrement qu'échanger, être en commerce avec d'autres disciplines, d'autres industries, dans pratiquement tous les domaines des humanités ou des sciences. Dans sa pratique comme dans sa réflexion théorique, le designer a la nécessité de l'enquête et de l'échange sur au moins deux plans. Au plan social et humain, d'abord, au sens où tout projet, qu'il porte sur un produit, l'espace public ou un dispositif, s'ancre dans un contexte ou une situation et l'oblige donc à fréquenter les sciences humaines et sociales : l'histoire sociale et l'économie politique, l'histoire des techniques, la sociologie, sans oublier la théorie critique. Ensuite, au plan de la recherche appliquée : le désigner s'informe naturellement des avancées en génie mécanique, en sciences des matériaux, en biologie, etc. Aujourd'hui plus que jamais, où les enjeux de l'écologie et du numérique, y compris l'IA, s'imposent à nous.

Bref, le designer a l'habitude de l'échange et de l'insertion dans des équipes interdisciplinaires. Ce qui change, c'est qu'il n'est plus le simple metteur en forme. Il est de plus en plus amené à interroger et formuler à son tour les hypothèses de travail pour sa propre discipline, quand il avait l'habitude de le faire pour sa pratique, ce qui n'est pas la même chose.

\section{《L La MSH aura ainsi permis d'accélérer l'installation d'une bistoire du design (...) 》〉}

- On comprend que síl y a encore un travail de conviction à faire, il ne s'adresse plus tant aux designers qu'aux autres chercheurs quant à l'intérêt pour eux de s'ouvrir davantage au design...

Absolument, et à cela, la promotion d'un axe historique peut contribuer, car en plus d'être utile aux jeunes designers et chercheurs, il permet de reconnaitre au design un point commun avec les autres disciplines, à savoir son historicité. 



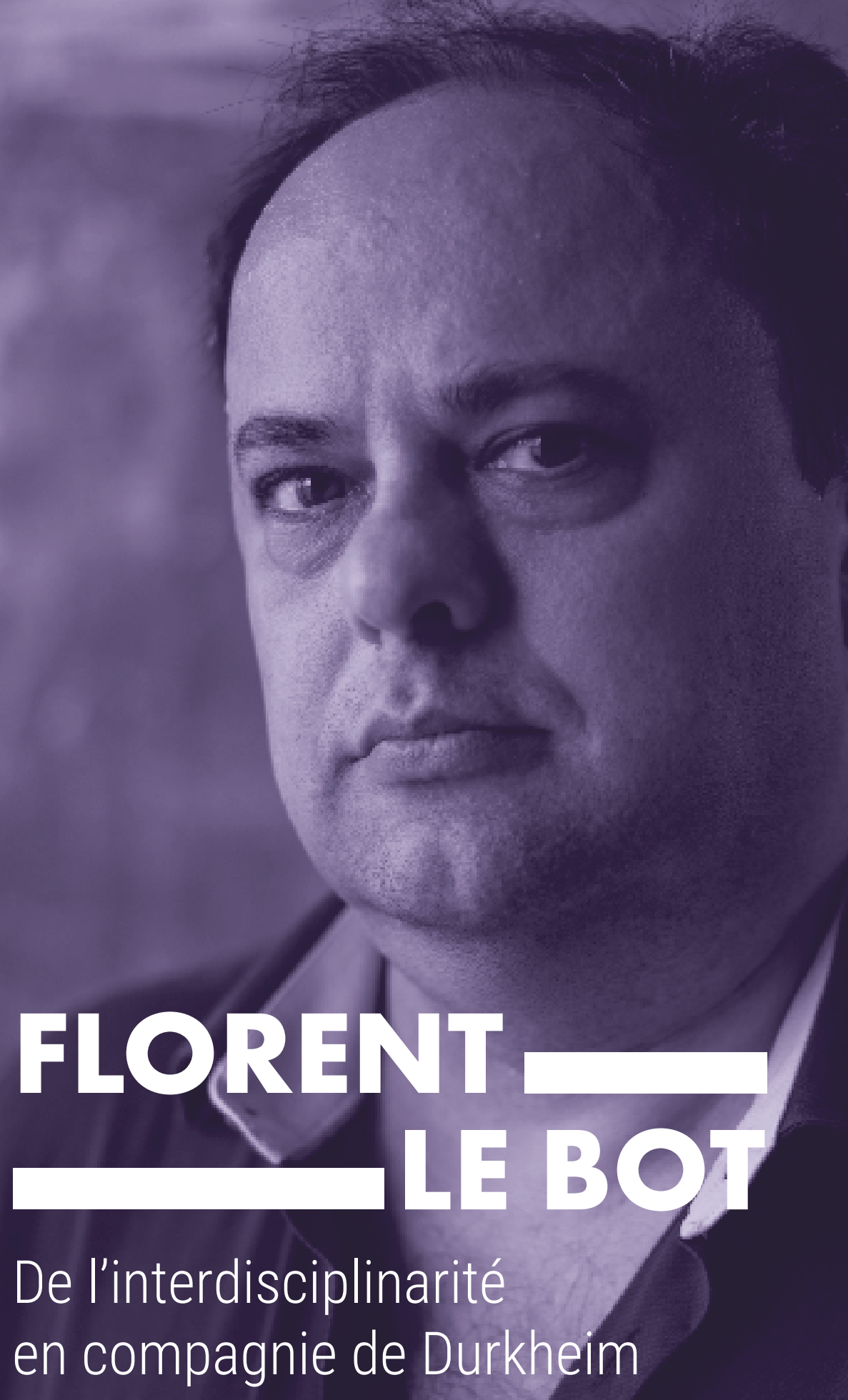


Enseignant-chercheur au laboratoire IDHES (UMR 8533), à l'Université d'Évry Val-d'Essonne (ENS Paris-Saclay, CNRS), Florent Le Bot est historien des mondes contemporains. Ses travaux portent sur les dynamiques historiques de l'économie. II est à l'initiative des Petits Déjeuners Durkheim, un cycle de conférences coorganisé avec la MSH Paris-Saclay, à la Bibliothèque Durkheim de I'ENS ParisSaclay. II est par ailleurs membre du comité de rédaction de l'une des deux revues scientifiques hébergées à la MSH Paris-Saclay, L'Homme \& la Société, pour laquelle il a récemment coordonné deux numéros consacrés à "L'HommeMachine».

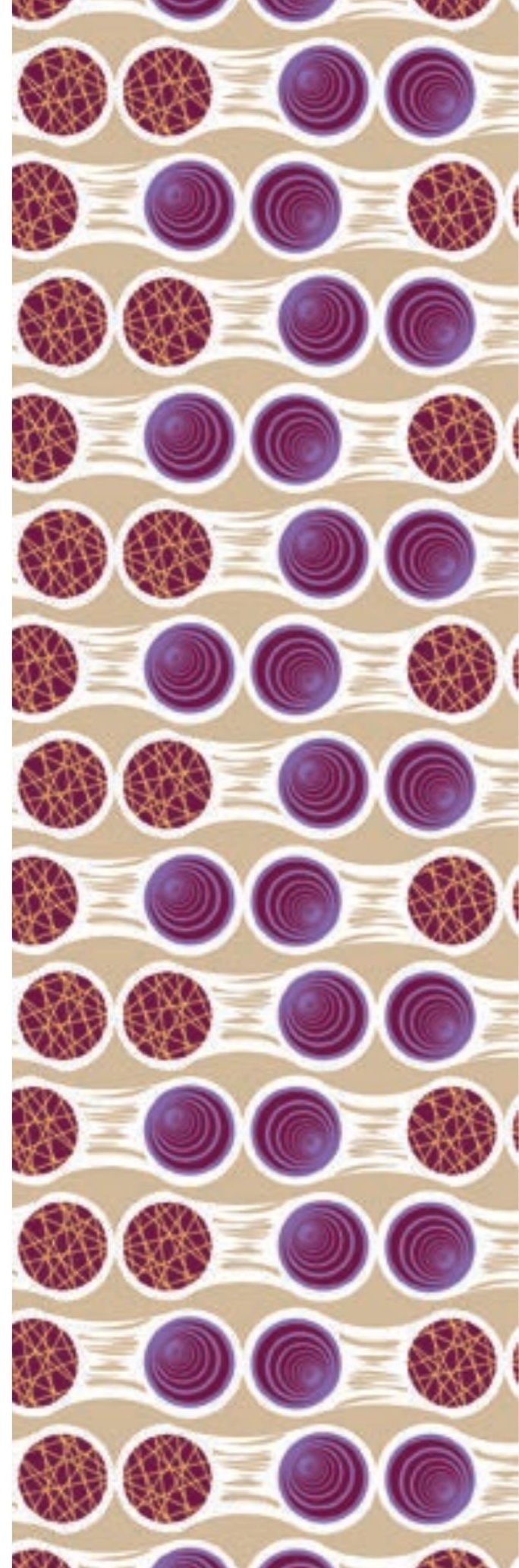




\section{Florent LE BOT \\ Enseignant-chercheur à l'IDHES Évry (UMR 8533) \\ Directeur adjoint de l'Inspé de l'académie de Versailles}

- Comment en êtes-vous venu à vous intéresser aux dynamiques territoriales dans une perspective bistorique?

Je travaillais sur le vol des biens juifs durant l'Occupation, plus précisément sur la spoliation des entreprises de la branche du cuir (tanneries, maroquineries, chaussures, ganteries), alors très nombreuses à l'époque. J'ai tout particulièrement porté mon attention sur le rôle joué par les patrons en situation de concurrence, dans ce processus de spoliation. Sources à l'appui, j’ai pu constater que la crise économique des années 1930, conjuguée à l'organisation duale de la branche, éclairait dans une large mesure les prises de position et les engagements des acteurs économiques dans les spoliations durant les années d'Occupation. Organisation duale, ai-je dit, au sens où se confrontaient deux catégories d'entreprises : d'une part, de grandes entreprises modernisées, rationalisées, taylorisées et solides financièrement (à l'image, par exemple, des entreprises de chaussures Bata, d'origine tchèque, ou André, propriété de patrons considérés comme juifs), d'autre part, des grappes d'entreprises, à la fois concurrentes et complémentaires, regroupées sur des territoires spécifiques : Fougères, Limoges, Roman, etc. Soit ce qu’il est convenu d'appeler des "districts industriels », depuis les travaux fondateurs de l'économiste britannique Alfred Marshall.

Frappées par la crise, les secondes en rendaient responsables les grandes entreprises, dénonçaient leurs pratiques concurrentielles en les 
jugeant déloyales, «importées de l'étranger», voire « juives». Les mêmes dénonçaient par la même occasion comme «parasites » les tous petits artisans étrangers notamment parisiens, souvent d'immigration récente et juifs.

Cette situation de confrontation de deux mondes de production distincts - le monde industriel des grandes marques et le monde interpersonnel des petites entreprises agglomérées, pour reprendre la terminologie de l'économiste Robert Salais et du géographe Michael Storper - m'a sensibilisé à la nécessité d'une approche pluridisciplinaire, celle que mobilise la science sociale dans toutes ses composantes - l'histoire, ma discipline d'origine, la géographie, déjà associée étroitement à la première, mais aussi l'économie, la sociologie, etc.

\section{《 (...) ce sont des chercheurs de l'ensemble de l'Université Paris-Saclay qui sont invités à venir présenter une publication récente, que ce soit une thèse, une HDR, un essai personnel ou collectif, etc. II}

- Ce qui vous prédisposait donc à participer à des projets labellisés par la $M S H$...

En effet. Depuis mon entrée dans le monde de la recherche, j'appartiens à un laboratoire, l'IDHE, créé en 1997, devenu depuis l'IDHES (pour Institutions et dynamiques historiques de l'économie et de la société). Comme son nom l'indique, il associe, au plan disciplinaire, histoire, économie, sociologie et, dans une moindre mesure, droit et géographie. Précisons que Robert Salais comptait parmi ses fondateurs, tandis que l'un de ses directeurs ne fut autre que le sociologue Claude Didry, qui a porté la MSH Paris-Saclay sur les fonts baptismaux.

- MSH qui a pour particularité d'assumer peut-être plus que d'autres un ancrage dans un territoire en adéquation avec vos terrains de recherche, puisque, à défaut d'être un district industriel, il participe à un cluster, une notion popularisée par Michael Storper, que vous évoquiez... 
En effet. J'ajouterai cette autre particularité qui tient à l'adossement de la MSH à une université, l'Université Paris-Saclay, qui est elle-même une fédération de plusieurs établissements d'enseignement supérieur et de recherche (les universités Évry Val-d'Essonne, Paris-Sud, Versailles Saint-Quentin-en-Yvelines, l'ENS Paris-Saclay, etc.) et d'organismes associés (CNRS, Inra, CEA, Inserm, etc.), qui participent fortement aux dynamiques économiques territoriales, au travers de la valorisation scientifique, des démarches partenariales avec des entreprises ellesmêmes implantées sur le territoire.

- Dans quelle mesure la MSH a-t-elle permis d'approfondir votre approche interdisciplinaire? L'occasion d'en venir aux Petits Déjeuners Durkbeim...

L'initiative de ces Petits Déjeuners en revient à Claude Didry, une rencontre d'ailleurs marquante dans mon parcours professionnel. Le principe en avait été imaginé dès 2012, dans la perspective de la création de la future Université Paris-Saclay. Il s'agissait de contribuer à faire se rencontrer les chercheurs en sciences sociales de l'ENS Cachan (aujourd'hui Paris-Saclay), autour de leurs travaux. La MHS Paris-Saclay nous a permis de franchir un pas supplémentaire puisque, désormais, ce sont des chercheurs de l'ensemble de l'Université Paris-Saclay qui sont invités à venir présenter une publication récente, que ce soit une thèse, une HDR, un essai personnel ou collectif, etc. Notre collègue invitée dispose d'une heure pour cela, puis vient le temps de l'échange, en présence éventuellement d'un·e discutant•e, spécialiste de la question. Ces échanges sont d'autant plus riches que les participants qui assistent aux Petits Déjeuners viennent d'horizons divers : y sont conviés aussi bien les chercheurs, les enseignants, que les étudiants et le grand public.

Depuis la saison 2019, les Petits Déjeuners Durkheim sont inscrits au programme de l'École doctorale SHS de l'Université Paris-Saclay, ce qui signifie que les doctorants de l'ensemble des établissements membres de cette école peuvent inscrire ce séminaire dans leur programme de formation. C'est ainsi que cette année, nous avons compté parmi les participants des doctorants en sociologie, en économie, en histoire, en science de l'éducation et même en théologie. 
Précisons que les séances sont filmées, captées en vidéo et disponibles sur la chaine YouTube de la MSH Paris-Saclay, ce qui sert pleinement l'ambition de ce séminaire, qui est de prendre part à la constitution d'une communauté de chercheurs en sciences sociales et, par ailleurs, de faire connaître les travaux de l'université auprès d'un plus large public.

- Est-ce à dire que les Petits Déjeuners Durkbeim répondent d'abord à un enjeu de diffusion et de communication?

À un enjeu de diffusion et de communication, mais aussi, j’insiste, de renforcement de l'interdisciplinarité. Il importe que les chercheurs de différentes disciplines prennent le temps de se parler au-delà de leurs disciplines. Non que les chercheurs ne se parlent pas déjà entre eux, mais, le plus souvent, c'est avec des collègues de la même discipline sinon de quelques autres, proches des leurs et toujours les mêmes, qui fréquentent les mêmes lieux, les mêmes colloques ou séminaires. Historiens et géographes, pour ne prendre que cet exemple, se parlent, mais sans doute ne le font-ils pas encore assez avec des chercheurs d'autres disciplines des sciences sociales. Au sein d'une même discipline, force est de constater des cloisonnements du fait des spécialisations. Pour ma part, étant historien contemporanéiste, je suis enclin à échanger d'abord avec des historiens du XIX ${ }^{\mathrm{e}}$ et $\mathrm{XX}^{\mathrm{e}}$. De même, avec les économistes, nos échanges portent sur des thématiques spécifiques.

Nos Petits Déjeuners Durkheim sont l'occasion d'élargir le spectre, en discutant d'ouvrages qui sont loin de nos champs respectifs. Â dessein, nos programmes sont vastes, ils vont des sciences et des techniques à la sociologie des sciences, en passant par le droit, l'histoire culturelle l'histoire de l'éducation, la géographie économique, etc.

- Mais alors pourquoi cette référence au sociologue Durkbeim, qui incarne une certaine vision de la sociologie, sinon des sciences sociales?

Tout simplement parce que les Petits Déjeuners se déroulent à la bibliothèque éponyme de l'ENS Paris-Saclay, créée en 2010. J'en profite pour préciser d'ailleurs qu'ils sont organisés avec le précieux concours de sa bibliothécaire, Nathalie Barnault, et d'Éric Valdenaire, de la MSH. Cela 
étant dit, le fait d'afficher le nom de ce sociologue dans l'intitulé de nos Petits Déjeuners n'est pas totalement fortuit non plus. Durkheim est une figure tutélaire de la science sociale, celle d'un chercheur qui a su marier différentes approches, en inscrivant la sociologie dans une perspective historique et quantitative. En ce sens, il manifestait déjà une volonté de croiser les disciplines, en intégrant des outils des sciences exactes.

- Quel autre apport a représenté pour vous la MSH?

La MSH héberge L'Homme \& la Société, une revue créée il y a plus de cinquante ans, en 1966. Grâce au concours décisif de l'éditrice AnneSophie Décriaud, elle a connu un nouvel élan en changeant de format et de présentation. Une illustration des moyens concrets mis à disposition des chercheurs pour aider à la diffusion de leurs travaux. Précisons que L'Homme \& la Société a pour sous-titre «Revue internationale de recherches et de synthèses en sciences sociales ». C'est dire si sa vocation est pluridisciplinaire et combien le soutien de la MSH se justifie. Son comité éditorial, auquel je participe, compte des sociologues, des économistes, des historiens, des spécialistes de littérature, des juristes, des philosophes, etc.

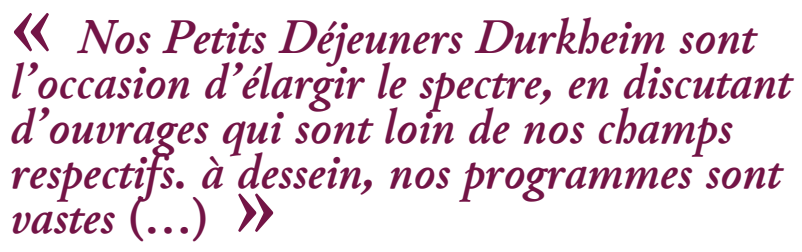

- Dans quelle mesure votre expérience de la MSH vous a-t-elle conforté dans l'idée que l'interdisciplinarité n'est pas qu'un mot d'ordre, qu'elle s'incarne dans des projets concrets propices à de réelles avancées, y compris en termes de contenu, sans nier l'intérêt de l'existence des disciplines constituées?

Des institutions comme la MSH sont capitales, en ceci qu'elles permettent de déployer des travaux recherche sur des terrains originaux et dans un esprit plus collaboratif. En ce sens, elle fonctionne à la manière d'un incubateur d'innovation, en permettant le développement de projets 
novateurs qu'on ne pourrait pas soumettre ailleurs, faute d'entrer tout à fait dans le cadre attendu par les évaluateurs. C'est dire si cette institution procure une nouvelle respiration aux chercheurs, en les plaçant hors de la pression de l'évaluation à laquelle ils sont soumis. 


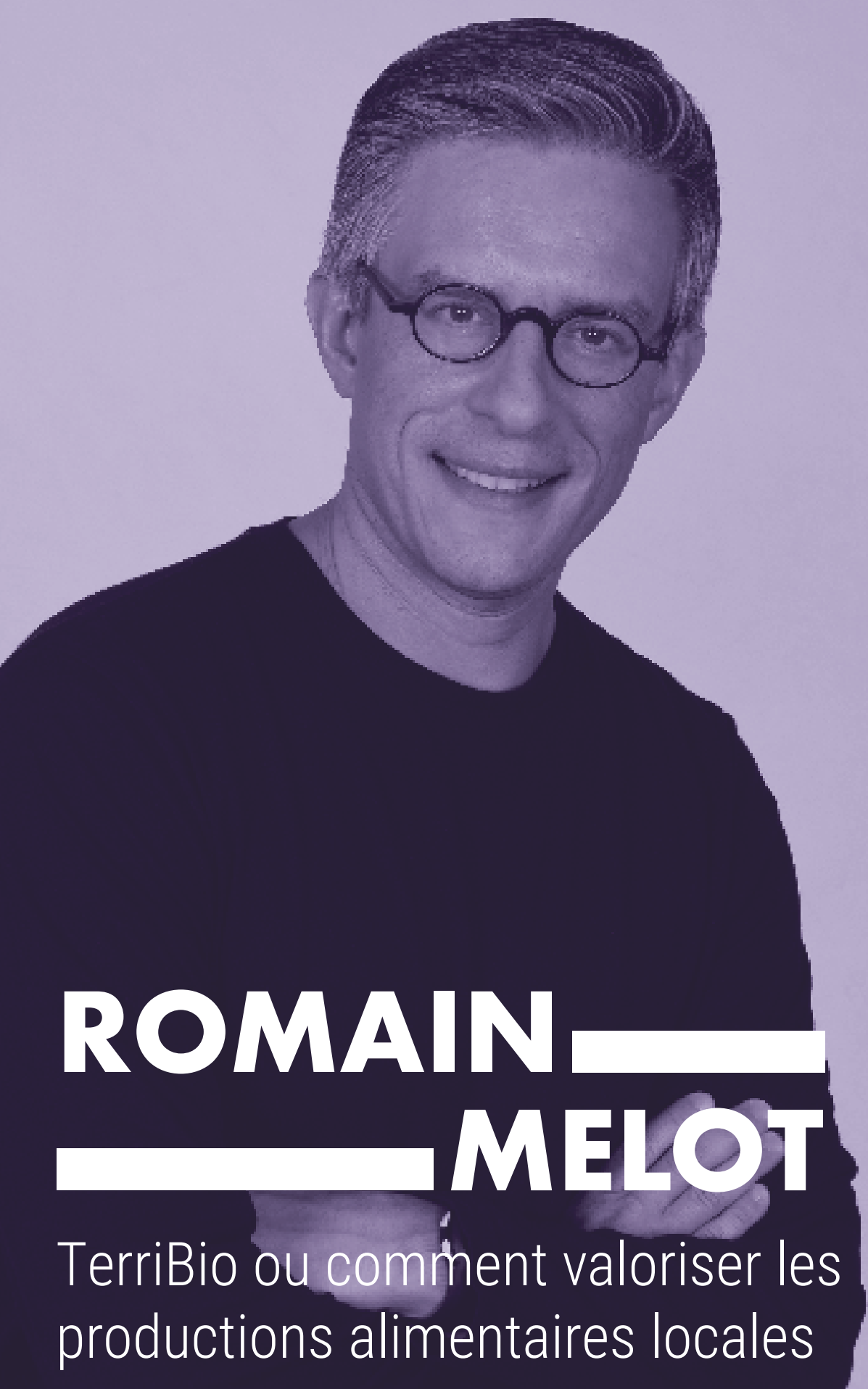


Directeur de recherche Inra à l'UMR SADAPT dont il codirige l'équipe Proximités, Romain Melot est sociologue, spécialisé dans l'étude dedifférents enjeux en lien avec les droits de propriété : l'aménagement du territoire, l'expropriation, l'environnement.

En 2017, il a été lauréat d'un appel à projets Émergence avec le projet TerriBio (pour TERRItoires d'interface et BIOdiversité urbaine en Île-de-France), centré sur l'analyse dela biodiversité urbaine comme vecteur de représentations sociales et comme objet pour l'action publique locale dans les projets d'aménagement. Plus récemment, en 2019, il a été lauréat, avec Emmanuelle Baudry (UMR Écologie, Systématique et Évolution ; Université Paris-Sud, CNRS, AgroParisTech), du plus important appel à projet de la $\mathrm{MSH}$, l'appel à projet Excellence, pour un projet qui s'inscrit dans la continuité du précédent, TerriBio Saclay.

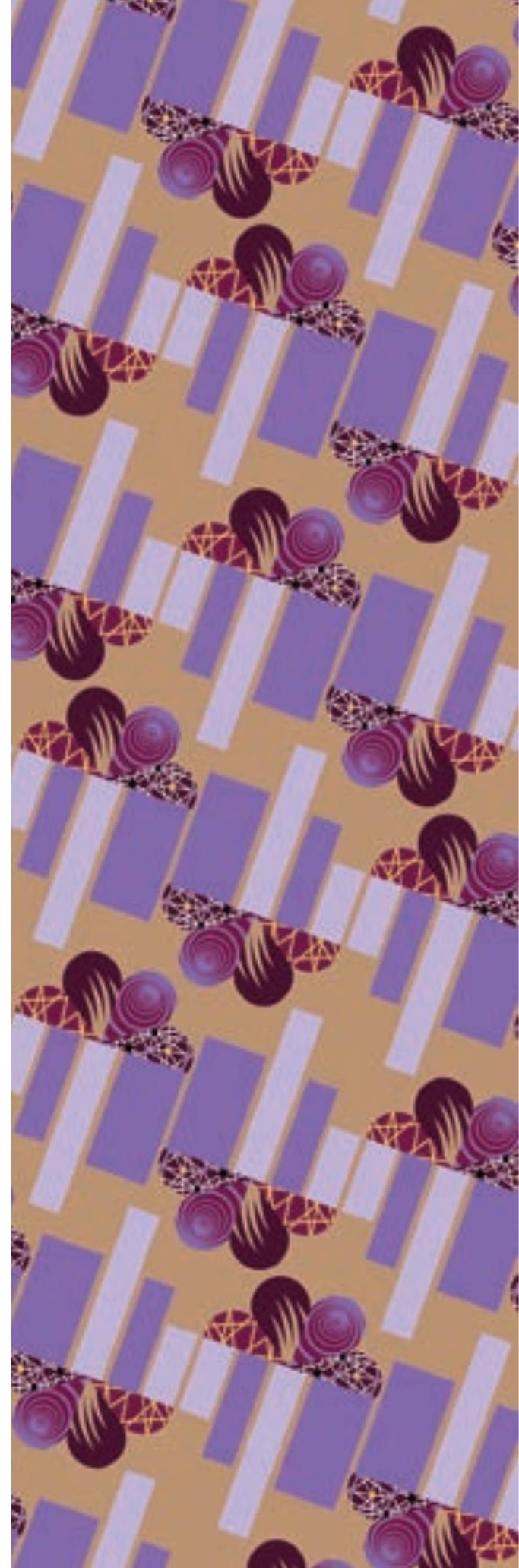




\section{Romain MELOT}

Directeur de recherche Inra/AgroParisTech, à I'UMR SAD-APT (UMR 1048) Codirecteur de l'équipe Proximités

- Un mot pour commencer sur la nature de vos travaux, au croisement de la sociologie et du droit...

Je suis en effet un sociologue du droit, au sens où je m'intéresse aux questions du foncier et des droits de propriété. Je traite de ces enjeux aussi bien sur le plan de la mise en ouvre du droit administratif (les contentieux de l'urbanisme et de l'environnement, les pratiques réglementaires en matière de planification) que sur le plan des litiges de droit privé (les contentieux du bail et de l'expropriation). En tant que chercheur à l'Inra, j'en suis venu à m'intéresser plus spécifiquement aux problématiques liées à l'usage des terres agricoles.

- Comme dans le projet TerriBio, pour lequel vous avez été lauréat de l'appel à projet Émergence. Pouvez-vous nous en rappeler la genèse et l'ambition?

Depuis 2016, dans le cadre du programme PSDR Île-de-France, je coordonne avec ma collègue Ségolène Darly, géographe à l'Université Paris 8, un projet intitulé « Cap-IDF ». Ce projet se propose d'étudier les différentes facettes de la production agricole - les exploitations professionnelles intervenant sur de grandes surfaces - mais aussi tout ce qui va se loger dans des interstices, entre les espaces bâtis et non bâtis, entre l'urbain et le rural - les micro exploitations des territoires périurbains, les jardins potagers de particuliers, etc. Retenu dans le cadre d'un appel à projets Émergence, TerriBio permet d'explorer plus 
précisément cette notion d'interstices urbains. Pour le sociologue du droit que je suis, ces espaces sont intéressants du fait même de leur ambiguïté, sinon complexité, mais aussi des questions de gouvernance et de gestion juridique qu'ils soulèvent : les uns sont la propriété d'une collectivité (commune ou intercommunalité), les autres de particuliers, qui n'ont pas les mêmes motivations au regard de la préservation de l'environnement. Les uns sont régis par des règles de droit public, leur gestion s'inscrivant dans des schémas d'aménagement (de type PLU ou PLUI), les autres (jardins de particuliers, espaces verts de copropriété...) relèvent du droit privé.

Pour y voir plus clair, nous produisons une cartographie détaillée de ces différents espaces. L'analyse de leur gouvernance et de la structuration de l'espace du territoire s'accompagne de recherches auprès des usagers du territoire, sur leur perception des aménités environnementales produites par les espaces ouverts. Des enquêtes ont porté également sur les trajectoires personnelles des utilisateurs du territoire, pour mieux cerner leurs motivations, leur rapport à la nature, ainsi que leur inscription dans les réseaux d'échange informels à l'échelle du voisinage.

\section{《 TerriBio permet d'explorer plus précisément cette notion d'interstices urbains. Pour le sociologue du droit que je suis, ces espaces sont intéressants du fait même de leur ambiguïté, sinon complexité, mais aussi des questions de gouvernance et de gestion juridique qu'ils soulèvent (...) I)}

- On devine l'enjeu au regard de la question de la densification de la ville...

En effet. C'est d'ailleurs pourquoi, avec ma collègue Ségolène Darly, nous travaillons étroitement avec des urbanistes et des architectes, qui ont une sensibilité aux sciences sociales. Je pense notamment à Béatrice Mariolle, qui a récemment codirigé l'ouvrage Densifier/Dédensifier avec Jean-Michel Léger et Béatrice Mariolle [Parenthèses, 2018], auquel Ségolène et moi avons d'ailleurs contribué. 
- À quelle suite a donné lieu ce premier projet?

Le projet TerriBio a d'abord fait l'objet d'un séminaire organisé en juin 2018 sur le thème "Jardins cultivés et biodiversité urbaine en Île-de-France ».

Puis, en 2019, j’ai été lauréat d'un appel à projet Excellence, pour le projet TerriBio Saclay, que je porte conjointement avec Emmanuelle Baudry, professeure à l'Université Paris-Sud et spécialiste d'écologie urbaine. Comme son nom le suggère, il s'inscrit dans le prolongement du précédent avec, cette fois, une focale sur le plateau de Saclay, un territoire intéressant s'il en est : en plus de faire l'objet d'une importante opération d'aménagement, il compte de nombreux espaces agricoles, naturels et forestiers préservés (préservés dans le cadre de la ZPNAF) et d'importantes zones pavillonnaires, parsemées d'espaces non bâtis et, donc, cultivables.

- Comment s'est déroulé le dialogue avec cette chercheure d'un tout autre domaine disciplinaire que le vôtre?

Il se trouve que nous nous connaissions déjà : nous étions responsables d'un projet PSDR (Pour et sur le développement régional). Pour mémoire, ce type de projet, porté par l'Inra, est décliné dans les régions, avec le soutien de celles-ci, en vue de mobiliser des chercheurs sur des enjeux de développement territorial et avec l'ambition de déboucher sur des préconisations, des résultats utiles aux acteurs socioéconomiques, les agriculteurs notamment. Emmanuelle coordonne également un projet de ce programme, intitulé "Dynamiques ", sur la biodiversité dans les espaces périurbains. Elle et moi avions déjà été amenés à discuter ensemble, mais nous ne serions probablement pas allés aussi loin dans notre collaboration s'il n'y avait eu la MSH de Paris-Saclay et cet appel à projet Excellence.

- Quel intérêt retenez-vous des appels à projet de la MHS comme ceux dont vous avez été lauréat?

Leur principal intérêt est de nous inciter, nous autres chercheurs en SHS, à nous rapprocher d'autres sciences, en l'occurrence, dans le cas des projets TerriBio, des sciences de la vie, et à dialoguer ainsi avec 
d'autres chercheurs ayant d'autres méthodes, d'autres concepts. En plus de favoriser une interdisciplinarité forte (entre SHS et sciences de la vie ou sciences exactes) et inter-établissement (entre l'Inra AgroParisTech, le CNRS, l'Université Paris-Sud et le Labex Basc), les appels à projet de la MSH ont encore ceci d'intéressant qu'ils incitent les SHS à être à l'initiative des projets et pas seulement à venir en appui de projets initiés par les autres sciences.

Un autre intérêt de ces appels à projet est leur déclinaison en fonction du degré de maturation de l'idée. Tandis que l'appel à projets Émergence permet à des collectifs de chercheurs de tester des idées, d'explorer un nouveau champ, de nouvelles thématiques, les appels à projets Maturation et Excellence font bénéficier de moyens plus importants, dès lors que les résultats sont probants. Cela permet d'inscrire des travaux de recherche dans la durée, en disposant de moyens à la mesure de la montée en puissance de ces travaux. Tandis que le projet labellisé Émergence a permis d'initier des enquêtes, le second projet, labellisé Excellence, permet d'en mener à une plus grande échelle, sur le plateau de Saclay.

\section{- Dans quelle mesure l'inscription de la MSH Paris-Saclay dans un territoire à forte composante agricole et maraîchère constitue un plus?}

Non seulement la MSH est inscrite dans un territoire qui correspondait aux enjeux de nos deux projets, mais encore elle est attachée à ce que nous poursuivions des recherches en lien avec les acteurs de ce territoire, le plateau de Saclay en l'occurrence. C'est ainsi que nous nous sommes rapprochés de Terre et Cité, une association qui œuvre notamment à l'animation de la ZPNAF (Zone de préservation des espaces agricoles, naturelles et forestiers). La collaboration a été d'autant plus fructueuse qu'elle a déjà une expérience dans l'implication de chercheurs dans ses propres projets. Nous l'avons impliquée dans la réalisation des enquêtes à base de questionnaires que nous souhaitions mener auprès de tous ceux qui concourent à la production alimentaire sur le plateau de Saclay, qu'ils soient professionnels ou non professionnels - exploitants agricoles, propriétaires de micro-fermes, de jardins potagers, partagés ou autres -, mais aussi des résidents du territoire, qui ne sont pas directement 
impliqués dans la production alimentaire, mais sont susceptibles d'avoir des choses à dire sur leur rapport avec ces espaces agricoles.

- À vous entendre, vous n'avez pas attendu la MSH pour pratiquer l'interdisciplinarité. Dans quelle mesure celle-ci vous a-t-elle non seulement permis de l'approfondir, mais encore convaincu de son intérêt?

Étant chercheur à l'Inra, j'ai effectivement l'habitude de travailler avec des agronomes en particulier. L'intérêt des appels à projet de la MSH est de m'avoir permis de travailler pour la première fois avec une écologue de l'urbain. Ces mêmes projets ont permis de solliciter d'autres personnes au parcours souvent atypique. Outre Emmanuelle Baudry, écologue de l'urbain, qui a elle-même travaillé avec des chercheurs au profil alliant plusieurs compétences, comme Anne-Caroline Prévot, une psychologue de l'environnement, je pense à Caroline Petit, une agronome et géographe, qui travaille sur les questions d'écologie territoriale et d'autonomie alimentaire locale ; ou à Pauline Frileux, de l'ENSP de Versailles, qui se définit comme "ethno-écologue » (elle est l'auteur d'un ouvrage récent sur les jardins pavillonnaires).

\section{《Leur principal intérêt est de nous inciter, nous autres chercheurs en SHS, à nous rapprocher d'autres sciences, (...) et à dialoguer ainsi avec d'autres chercheurs ayant d'autres méthodes, d'autres concepts}

- Quels sont les résultats les plus significatifs produits par ces projets?

Il est encore trop tôt pour communiquer dessus. Ce que l'on peut d'ores et déjà dire, c'est que le protocole des enquêtes directes par questionnaires que nous avons construit en commun se révèle a priori un bon support à une approche interdisciplinaire, en ce sens qu'il permet d'aborder aussi bien les pratiques que les représentations ou encore les trajectoires de vie. Autant de choses qui intéressent aussi bien les sociologues que les géographes. Les enquêtes ont aussi pour objectif de dresser un inventaire de la biodiversité présente sur le territoire, les pratiques culturales, etc. 
D'autres choses susceptibles d'intéresser cette fois les écologues, les agronomes et les autres spécialistes des sciences de la vie.

Je précise que notre projet permet de traiter d'un sujet d'actualité, à savoir l'entrée en vigueur, depuis le début de cette année, de la loi interdisant aux particuliers, propriétaires de jardins potagers, d'utiliser les pesticides. Or, souvent, dans les territoires périurbains, les jardiniers amateurs ont pour voisins des agriculteurs. Naturellement, les uns et les autres échangent sur l'impact de cette interdiction. Pour le sociologue que je suis, il est intéressant d'observer les conséquences que peuvent avoir ces changements contraints de pratique sur les échanges de voisinage avec les agriculteurs des environs. 


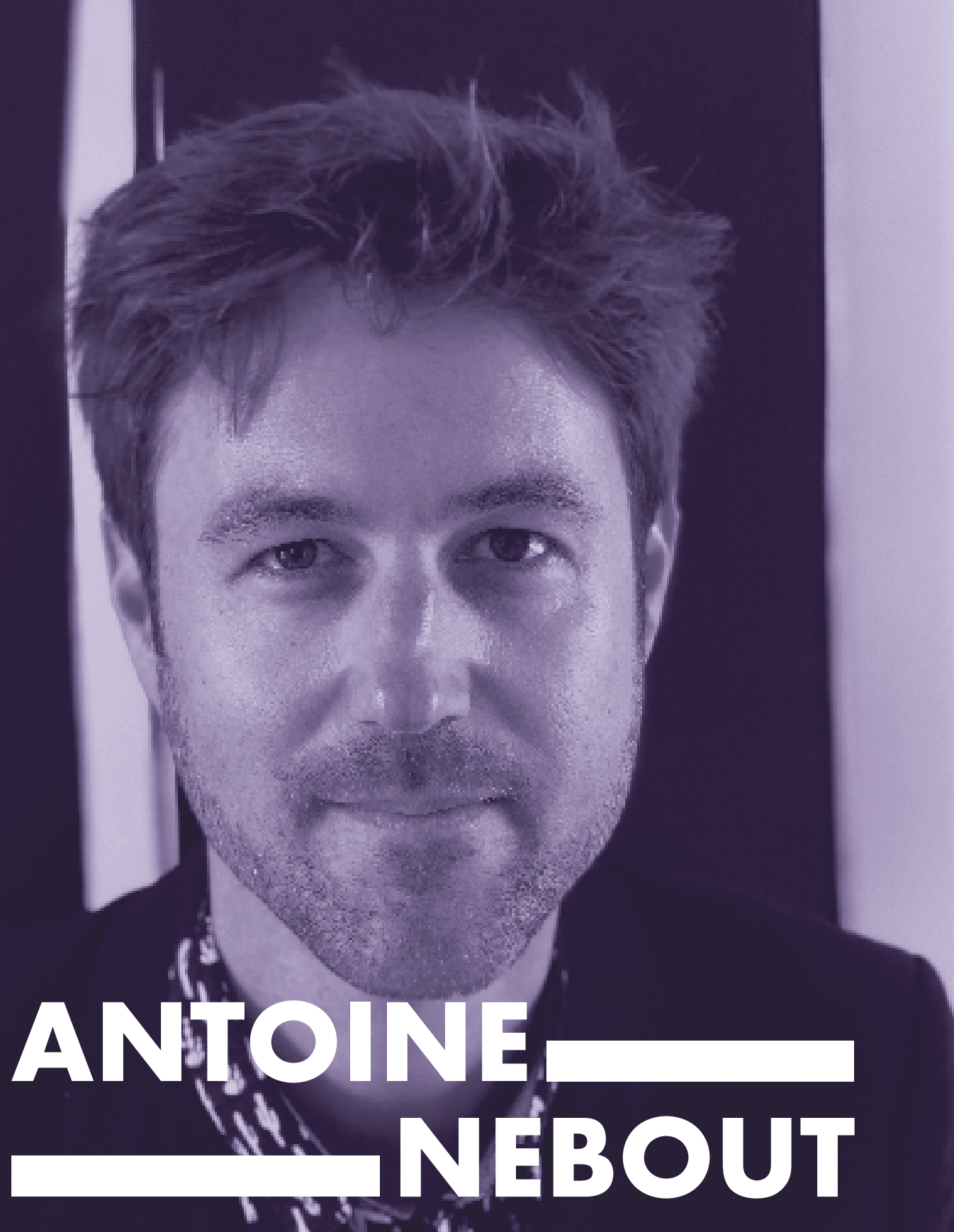

Quand des lécenomistes se penchent sur nos assiettles 
Chargé de recherche Inra à I'UR Alimentation et Sciences sociales (Aliss), Antoine Nebout est économiste. II s'intéresse notamment aux questions de risque, d'information du consommateur et aux questions relatives à l'essor du e-commerce.

En 2017, il a été lauréat d'un appel Maturation pour le projet $P$ sychofood, qui se propose d'étudier les déterminants psychologiques des comportements alimentaires. Objet d'un grand colloque final à la MSH en mai 2019, il connaît un prolongement au travers d'un autre projet, BEHealth, lauréat d'un appel à projet générique de l'ANR en juillet 2019.

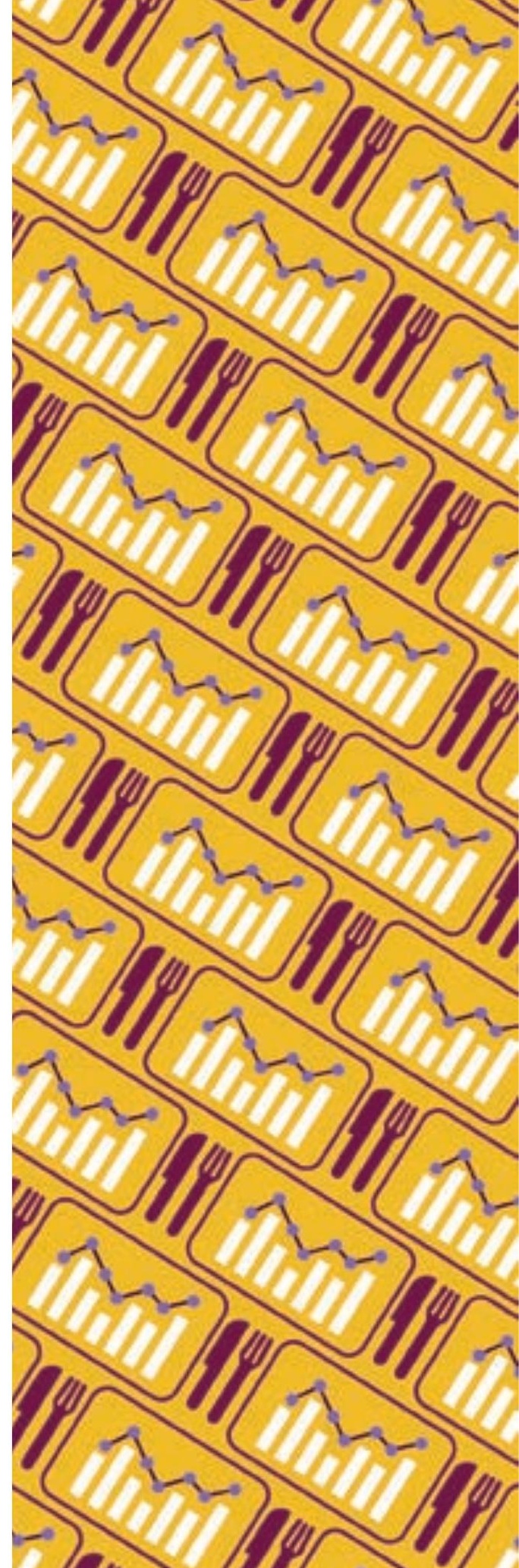




\section{Antoine NEBOUT}

Chargé de recherche Inra/AgroParisTech, à l'UR Aliss (UR 1303)

- Comment l'économiste de formation que vous êtes en est-il venu à étudier les comportements individuels?

Après deux années d'études à l'Ensae, qui forme les agents de la statistique publique, mais également des statisticiens-économistes du secteur privé, j’ai fait un master d'économie classique, à la London School of Economics. J'ai complété mon cursus avec un master en sciences cognitives (Cogmaster, EHESS), car je souhaitais m'ouvrir à d'autres disciplines mettant l'accent sur le comportement humain et la manière dont les individus prennent des décisions. La thèse sur la prise de décision dans le risque, l'incertitude et le temps que j'ai poursuivie au CNRS s'inscrivait bien dans la discipline économique, mais en s'appuyant sur la théorie de la décision, qui se trouve être à l'interface de plusieurs disciplines : l'économie, donc, mais aussi la psychologie et la philosophie. Naturellement, je me suis spécialisé en économie dite comportementale, qui s'emploie à comprendre en quoi les individus se comportent différemment de ce qui est attendu dans le modèle classique de l'bomo ceconomicus, censé agir dans le sens d'une optimisation de ses intérêts et de son utilité. Parmi les premiers à s'être intéressés à cette approche, il y eut Maurice Allais, premier prix Nobel d'économie français, les psychologues Amos Tversky et Daniel Kahneman, et les économistes Herbert Simon et Richard Thaler. 
- Comment en êtes-vous venu à vous intéresser plus spécifiquement aux comportements alimentaires?

Dans le cadre de ma thèse, je faisais de l'économie expérimentale, mais sans lien direct avec les comportements réels : je testais en laboratoire des modèles sophistiqués de décision, sur des populations d'étudiants d'universités. Cette approche de positivisme logique n'avait pas pour objectif d'expliquer des comportements réels, mais de mesurer les paramètres de ces modèles à partir de situations de choix stylisées. Lors de mon postdoctorat à l'Inserm, j'ai souhaité utiliser ces mesures pour expliquer les décisions médicales de médecins. Puis, après avoir intégré l'Inra et son laboratoire Aliss qui, comme son acronyme l'indique, s'intéresse à l'alimentation au regard des sciences sociales, j'ai entrepris d'appliquer les outils de l'économie comportementale à ce domaine, pour mieux éclairer les décisions en matière d'alimentation.

\section{$\ll$ (...) ce projet nous a permis de réunir des chercheurs en économie comportementale et en épidémiologie de la nutrition 》}

\section{- Du point de vue des seuls consommateurs?}

Dans le domaine de l'économie de l'alimentation, on distingue schématiquement deux catégories d'études. D'une part, celles qui mettent l'accent sur l'offre, en l'occurrence celle des entreprises de l'industrie agroalimentaire - soit les études en économie industrielle, qui portent sur les conditions de la concurrence, les stratégies industrielles, ... D'autre part, celles qui s'intéressent davantage à la demande des consommateurs, à la manière dont ils prennent leurs décisions d'alimentation, qu'elles portent sur l'achat ou le régime. Deux approches que mon laboratoire couvre. Pour ma part, c'est dans la seconde que je m'inscris.

- Les décisions relatives aux comportements alimentaires posent-elles des défis particuliers à l'économie comportementale?

Si leur étude soulève un défi particulier, c'est celui de pouvoir disposer de données suffisamment fiables sur les comportements alimentaires des 
individus. Des économistes utilisent des données récoltées par des sociétés privées spécialisées, en scannant tous les produits achetés par un panel de ménages, lesquels sont rémunérés en contrepartie. Ces données ont certes le mérite d'exister, mais elles ne portent que sur les comportements d'achat, sans permettre de décrire l'intégralité des comportements alimentaires. Pour notre part, et c'est tout l'enjeu du projet Psychofood que je supervise, nous nous appuyons sur un échantillon représentatif de la population française ; les questionnaires que nous adressons aux personnes les invitent à décrire précisément leur régime alimentaire complet : les catégories d'aliments qu'elles consomment, la fréquence de leur consommation sur une année, etc. Il s'agit là de données déclarées, avec tous les risques de biais qui peuvent en résulter. Le projet PsychAlim (CNRS/Inra) auquel je participe également a donc pour objectif d'évaluer l'impact du mode de collecte des données sur leur qualité. Notre défi de long terme est de parvenir à nous assurer d'une correspondance stricte entre le régime déclaré et le régime réel des individus. À terme, il est probable que de nouvelles technologies d'information et de communication soient mobilisées dans les projets de recherche.

- Dans quelle mesure la MSH vous a-t-elle permis d'approfondir vos thématiques de recherche actuelles?

En me donnant des moyens matériels et financiers pour mener à bien le projet Psychofood, que je viens d'évoquer. Retenu en 2017, dans le cadre de l'appel à projets Maturation, il nous a permis d'avancer dans l'étude des déterminants socioéconomiques, démographiques et psychologiques des comportements alimentaires des Français. Nous avons pu procéder à partir d'un questionnaire de fréquence alimentaire adapté de celui [qui est] développé par les équipes du Centre de recherche en épidémiologie et santé des populations (CESP) de l'Inserm et que nous avons soumis à un échantillon représentatif de la population française (composé de 2600 personnes invitées à répondre via des tablettes tactiles). Par nature interdisciplinaire, ce projet nous a permis de réunir des chercheurs en économie comportementale et en épidémiologie de la nutrition. Sur un plan plus matériel, le financement de la MSH nous a donné les moyens 
d'organiser des réunions et de participer à des conférences. Les premiers résultats ont notamment été présentés lors d'un workshop qui s'est tenu à la MSH Paris-Saclay au printemps.

D'une certaine façon, Psychofood m'aura également permis de faire mes gammes avant de me lancer dans un projet encore plus ambitieux : le projet BEHealth, lauréat d'un appel à projets JCJC de l'ANR. Il s'agit là encore de procéder à une collecte de données, au sein d'une cohorte épidémiologique, avec la possibilité d'appareiller nos données avec celles de la statistique publique et de l'assurance maladie. On ajoute ainsi une dose d'objectivité des plus précieuses, notamment dans le cadre de l'étude médicale de l'impact de l'alimentation et des comportements à risque sur la santé.

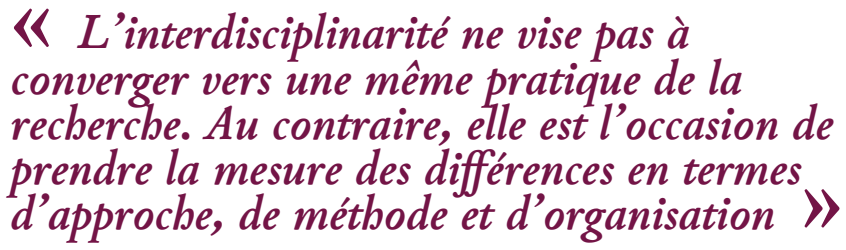

- Dans quelle mesure le Big Data change-t-il la donne?

Au sein du laboratoire Aliss, on utilise déjà des bases de données issues de recherche en Big Data. Des collègues s'intéressent en particulier aux nouvelles méthodologies qu'on pourrait développer à partir des données collectées via nos applications et nos smartphones ou sur le Web. Des projets sont en cours. Cependant, dans le cas de Psychofood comme de BEHealth, nous avons pris le parti de rester dans le cadre relativement classique, de cohortes et d'échantillons représentatifs à l'échelon national.

- Dans quelle mesure votre expérience de la MSH vous a-t-elle fait évoluer dans votre vision de l'interdisciplinarité?

Au cours de mon cursus, j'ai toujours eu une curiosité personnelle pour d'autres disciplines que la mienne et eu envie de faire dialoguer des disciplines ayant pour point commun la prise de décision individuelle. Dès la fin de mon postdoctorat, j'ai souhaité utiliser les outils de l'économie 
comportementale pour étudier l'alimentation. J'avais donc à cœur de me rapprocher d'autres équipes qui étudiaient l'alimentation au niveau de la population et notamment des épidémiologistes de la nutrition. J'avais repéré l'existence du panel ELIPSS - un outil mis en place dans le cadre d'un EquipEx à Sciences Po - qui permettait d'adresser via une tablette un questionnaire de 30 minutes à un échantillon représentatif de la population française. Ce que nous avons pu faire en répondant à l'appel à projets ELIPSS avec l'équipe interdisciplinaire du projet Psychofood.

Je n'ignore pas les freins auxquels se heurte la pratique de l'interdisciplinarité. Mais, comparé à ceux de maître de conférences ou d'assistant professeur, mon statut de chercheur me permet de disposer de plus de liberté et du temps nécessaire. Étant moins soumis, à court terme, à la pression de la publication et de l'enseignement, je peux assumer mon approche interdisciplinaire. Mettre en place des projets avec des chercheurs d'autres disciplines que la sienne reste quelque chose de compliqué et de chronophage. C'est dire aussi l'importance du rôle de la MSH qui contribue largement à faciliter la tâche des chercheurs sur le plan logistique.

- Ne vous beurtez-vous pas à des différences de méthode voire de fonctionnement?

Si, bien sûr. L'interdisciplinarité ne vise pas à converger vers une même pratique de la recherche. Au contraire, elle est l'occasion de prendre la mesure des différences en termes d'approche, de méthode et d'organisation. À l'occasion de Psychofood, j'ai pu observer que mes collègues épidémiologistes n'avaient pas les mêmes modes de fonctionnement que nous autres économistes, notamment dans les modalités de rétribution des personnes impliquées dans les projets. En économie, quand nous devons faire des études statistiques, nous nous en remettons à un des auteurs du papier, un statisticien/économètre, qui exploitera les données du projet. En épidémiologie, il faut financer le recrutement d'un ingénieur d'étude ou de recherche qui se chargera des analyses statistiques. Des différences de fonctionnement, qui peuvent engendrer parfois des incompréhensions. Il faut le savoir. 
De même, les modes de publications sont différents : les chercheurs en SHS ont l'habitude de publier des papiers relativement longs, d'au moins 20 pages, la publication s'inscrivant dans un processus de trois à cinq ans. Les épidémiologistes publient, eux, des articles relativement plus courts, se focalisant sur des résultats très précis ; leur publication dans des revues scientifiques est plus rapide et parfois payante. Bref, à chaque discipline, sa sociologie de la recherche, qu'il faut prendre le temps de découvrir. L'interdisciplinarité, c'est aussi cela : un nécessaire apprentissage des fonctionnements des autres chercheurs et équipes, et c'est en cela que c'est intéressant.

- Qu'en est-il de l'économiste de formation que vous êtes? Vous définissez-vous toujours ainsi après plusieurs années d'expériences de l'interdisciplinarité?

Je ne suis pas sûr de m'être jamais défini comme un économiste de formation. Comme je le rappelais, mes travaux ont été d'abord marqués par la théorie de la décision, laquelle n'est pas l'apanage de la seule science économique - elle intéresse aussi la psychologie et la philosophie. Au sein d'Aliss, je me retrouve au milieu de chercheurs, qui s'intéressent à l'économie industrielle, aux prix, ... Pour ma part, c'est bien au comportement individuel que je m’intéresse. Si, donc, je devais me définir, ce serait comme économiste comportemental, sinon comme chercheur en sciences comportementales, lesquelles sont par nature interdisciplinaires. 

Chargée de recherche CNRS en linguistique, Ioana Vasilescu travaille au Laboratoire d'informatique pour la mécanique et les sciences de l'ingénieur (Limsi, un laboratoire CNRS, associé à I'Université Paris-Sud), sur le traitement automatique de la communication parlée.

En 2017, elle a été lauréate d'un appel à projets Émergence avec le projet "HistorlA ", qui se propose d'explorer les changements sonores avec l'intelligence artificielle. La même année, ce projet faisait l'objet d'un colloque sur le thème " Linguistique et Big Data ". L'année suivante, elle était lauréate d'un appel à projets Maturation pour le projet " HistorlA.2 ». Destiné à approfondir l'étude de l'évolution des langues avec les moyens de l'intelligence artificielle, il a notamment contribué à la réalisation et la mise en ligne d'un Atlas sonore des langues régionales.

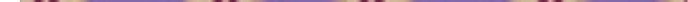




\section{Ioana VASILESCU}

Chargée de recherche CNRS au Limsi (UPR 3251)

- Pour commencer, pouvez-vous rappeler comment vous en êtes venue à ce domaine de recherche au croisement de plusieurs champs d'études, la linguistique et le numérique?

J'ai commencé par poursuivre des études en langues et littératures roumaine et française à Bucarest. Aussi, quand je suis arrivée en France, en 1996, ce fut avec un bagage à dominante philologique : en Roumanie, on privilégiait l'histoire des langues latines, la phonétique historique et la lexicographie dans une perspective étymologique (on s'attachait à étudier l'origine, l'évolution du sens et de la forme des mots). J'en ai gardé une passion pour l'évolution des langues et les lois qui la gouvernent. C'est tout naturellement que je me suis inscrite en Sciences du langage à l'université Lyon 2.

Mon ouverture sur les enjeux du langage automatique intervient à la fin des années 1990. En 1998, je me suis inscrite au master Ingénierie des langues proposé par l'Aupelf [Association des universités partiellement ou entièrement de langue française]. Ce master pour le moins exotique à l'époque (il était parmi les premiers à proposer ce croisement entre ingénierie et linguistique) a été pour moi comme une révélation. Des cours mettaient en relation langue et traitement informatique. Pour la première fois, j’ai eu un aperçu à la fois du fait qu'on étudiait la langue dans ce cadre applicatif et qu'il pouvait y avoir un impact de la variation langagière sur la performance de ces outils. Parmi les cours proposés, quelques-uns se tenaient au Limsi (les autres avaient lieu à 
Marne-la-Vallée et à l'ISTN du CEA). J'ai eu grand plaisir à fréquenter ce laboratoire, où je ne demandais qu'à avoir l'opportunité de travailler.

Depuis, beaucoup de chemin a été fait en matière de linguistique et de traitement automatique. Pour ma part, je n'ai eu de cesse de m'intéresser aux systèmes issus du monde de l'ingénierie, non pas tant pour en devenir une spécialiste, mais pour faire de la recherche en linguistique. Mon sujet de thèse de doctorat porta sur la comparaison entre humains et systèmes automatiques dans l'identification des langues. Puis j'ai eu l'immense chance de décrocher un poste CNRS au... Limsi.

\section{- Sur quoi portèrent vos travaux de recherche?}

J'ai poursuivi mes recherches sur la comparaison entre les humains et les systèmes automatiques, puis en portant davantage mon attention aux erreurs des systèmes de reconnaissance vocale et à la variation inhérente à la langue parlée. Progressivement, j'en suis arrivée à m’intéresser à la relation de la variation captée par ces systèmes comparée à l'évolution des langues, lorsqu'un élément de variation de stabilise et devient fait spécifique à une langue, adopté par tous les locuteurs. C'est ainsi qu'a pu s'instaurer un pont durable entre ma formation première, philologique, et le traitement automatique.

\section{- Quel défi cela représente-t-il en termes d'interdisciplinarité?}

Les défis sont multiples : tout d'abord, travailler avec des chercheurs venant d'autres horizons disciplinaires ou professionnels que le sien suppose de trouver un "langage » commun. Il s'agit aussi de définir un thème de recherche qui puisse intéresser chacun. À l'Université ParisSaclay, nous ne sommes que trois linguistes (tous chercheurs CNRS de la section 34, linguistique). C'est dire s'il nous faut trouver notre place. Au sein du Limsi, j'ai eu l'opportunité de travailler dans une équipe formidable en Traitement du langage parlé (TLP) avec des collègues dont les intérêts scientifiques allaient bien au-delà de la seule performance des systèmes. Nous avons pu développer des sujets portant à la fois sur le fonctionnement des langues et sur la relation entre spécificités linguistiques et performances automatiques. J'ai pu obtenir des résultats 
fiables et pertinents pour mieux comprendre des faits linguistiques jusqu'alors étudiés majoritairement sur des corpus de taille restreinte, dits « de laboratoire».

Un autre défi est propre à la reconnaissance de nos travaux au sein des sciences humaines. Ce n'est que depuis peu que les approches que je développe et qui impliquent de grands corpus et l'utilisation de systèmes automatiques en tant que véritables outils linguistiques sont reconnues par mes collègues linguistes comme véritablement "linguistiques». Reste qu'il demeure difficile d'obtenir des financements purement SHS.

- En quoi le Big Data a-t-il cbangé la donne par rapport à ce que pouvait recouvrir jusqu'ici l'ingénierie linguistique?

Lorsque je suis arrivée en France, mon laboratoire de recherche me mettait à disposition magnétophone et logiciels dont la licence coûtait cher, pour les besoins de mon travail de thèse. Créer et analyser son corpus de "laboratoire » était donc laborieux. Désormais, on dispose de quantités impressionnantes de données langagières, écrites et orales, sur Internet. À défaut, il est relativement facile de les produire, selon le besoin expérimental, et de les exploiter avec des outils libres. Un étudiant en master peut enregistrer les données de son travail de mémoire avec un simple smartphone et trouver des logiciels libres en tout genre sur Internet pour analyser ses données.

On ne peut prétendre faire abstraction de cette richesse : ces données existent, nous pouvons y avoir accès et fonder nos réflexions linguistiques théoriques sur l'existant.

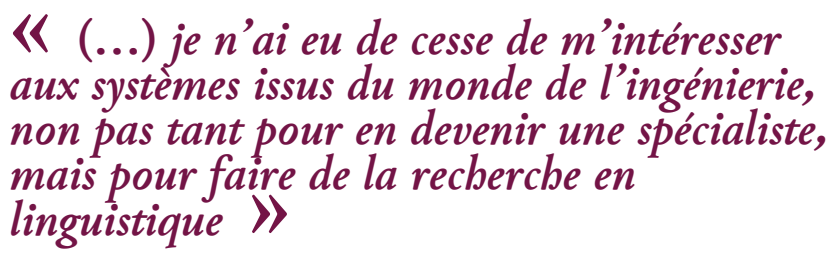




\section{- Sans incidence d'un point de vue méthodologique?}

Si. Passer du corpus restreint, de laboratoire, à des données massives implique une autre technique de travail, une autre manière d'aborder les faits de langue, et, donc, des résultats d'un type différent. Les grands corpus et les méthodes de travail scientifique associées enrichissent et valident des approches linguistiques précédentes. Il ne s'agit pas de nier tout le passé des données dites de « laboratoire », mais de reconnaître une nouvelle manière de faire de la linguistique. De ce point de vue, le Limsi a eu un rôle pionnier. Dès les années 2000 , mes collègues ont proposé des études sur la variation linguistique en s'appuyant sur les grands corpus utilisés pour entrainer les systèmes de reconnaissance vocale. Précisons cependant que grand corpus ne veut pas forcément dire Big Data. Celuici signifie une absence totale de maîtrise des caractéristiques du corpus. Or, en ce moment, nous utilisons plutôt des centaines d'heures - ce qui est déjà beaucoup - et nous savons typer les caractéristiques de nos données (journalistiques, conversations spontanées etc.).

- Vous avez été lauréate de plusieurs appels à projets de la MSH, notamment pour les projets HistorIA (appel à projets Émergence 2017) et HistorIA 2 (appel à projets Maturation 2018). Dans quelle mesure ont-ils contribué à l'approfondissement et l'élargissement de vos recherches?

Pour mémoire, le premier, HistorIA vise à mieux appréhender les mécanismes acoustiques et articulatoires qui régissent les changements sonores, à partir de données synchroniques, et ce en faisant appel à des outils qui relèvent de l'intelligence artificielle, notamment des systèmes de reconnaissance vocale. Il fait pour cela appel à de grands corpus oraux investigués avec des moyens numériques et statistiques. Ce projet a donné lieu en novembre 2017 à un colloque sur le thème «Linguistique et Big Data».

HistorIA.2 vise à mener plus loin cette réflexion et à approfondir l'étude de l'évolution des langues avec les moyens de l'intelligence artificielle à partir d'une exploitation de données massives multi-sources (synchroniques, diachroniques, dialectales). Ce projet a notamment contribué à la réalisation et la mise en ligne de l'Atlas sonore des langues 
régionales, dont les résultats permettent de répondre à un triple objectif : la sauvegarde du patrimoine linguistique, la validation de théories et le développement du machine learning pour la reconnaissance vocale.

S'il y a un premier enseignement à tirer de ces projets, c'est que les systèmes automatiques ne sont pas seulement des outils permettant de pré-traiter des données linguistiques, mais de véritables instruments de recherche permettant d'aller au plus profond des questions que les linguistes «classiques» se posent : comment évoluent les langues ? Comment d'une variation observable à un instant $\mathrm{t}$ on arrive à des faits linguistiques stables, à des changements linguistiques accomplis?

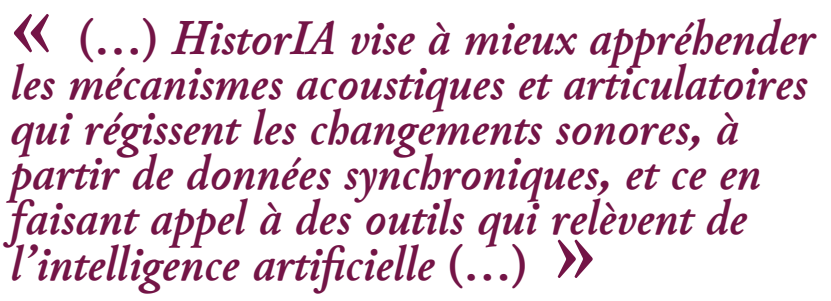

- Dans quelle mesure la MSH aura-t-elle permis de faire contribuer la linguistique aux bumanités numériques que, pour votre part, vous enseignez à Paris 3 Sorbonne Nouvelle - ENEAD?

Comme vous le savez, la question des humanités numériques est vaste et encore ambiguë. Il reste encore beaucoup à explorer dans ce domaine, en gardant à l'esprit qu'il s'agit d'un enjeu sociétal. Je pense que nous sommes tous d'accord pour considérer que ces «humanités numériques » doivent rimer avec inter- et pluridisciplinarité. Elles appellent la valorisation d'outils informatiques pour le stockage et l'analyse des données spécifiques aux humanités. Pour ce qui est de la linguistique, la MSH Paris-Saclay peut avoir un rôle phare, en montrant qu'il y a des opportunités de collaboration féconde entre elle et les technologies du numérique. Si les activités " historiques » du laboratoire Limsi concernent d'abord le traitement automatique des langues, les projets labellisés par la MSH ont apporté la démonstration que la perspective inverse - une approche des systèmes automatiques des langues à partir des questions que se posent les linguistes - est non seulement possible, 
mais encouragée dans l'environnement de recherche de Paris-Saclay. C'est d'ailleurs la volonté du laboratoire Limsi que de mettre lui-même en avant une approche interdisciplinaire des langues.

- On mesure l'intérêt des plateformes numériques, mais aussi d'opérateurs de la télécommunication et autres pour vos travaux de recherche. Comment apprébendez-vous leur valorisation?

Mes premiers travaux avaient la valorisation pour finalité. Je cherchais à mobiliser la linguistique pour améliorer les systèmes automatiques et les rendre plus viables. Désormais, ils consistent davantage à montrer comment des outils et des méthodes qui viennent d'un domaine applicatif (celui de l'ingénierie linguistique, donc) peuvent être récupérés par la linguistique classique. Concrètement, je me sers des systèmes automatiques pour mesurer la variation dans les langues via des paramétrisations spécifiques. Ils permettent de traiter des centaines ou des milliers d'heures d'enregistrements audio en très peu de temps et, de ce fait, offrir une vision très globale d'un motif de variation orale.

Les réponses qu'on obtient, si elles ne débouchent pas toutes seules sur des logiciels ou des brevets, contribuent à améliorer ces systèmes en intégrant cette dernière (via ce qu'on appelle les dictionnaires de prononciation). Elles permettent ainsi de réduire les erreurs de transcription automatique. Elles sont évidemment précieuses à la recherche fondamentale sur les langues et leurs évolutions. 


\section{Ć́니 A ZOLNSKI \\ Un fructueux dialogue entre droit et infornatique}




\section{Célia ZOLYNSKI \\ Professeure des universités à l'Université Panthéon-Sorbonne Membre de l'IRJS}

- Comment la juriste que vous êtes en est-elle venue à se placer à l'interface du droit et du numérique?

La thèse que j'ai faite il y a maintenant plus de quinze ans - je l'avais commencée à la fin des années 1990 - traitait de la question de la protection du droit d'auteur : je m'intéressais particulièrement à l'intervention du législateur dans la société de l'information à l'heure d'Internet. De nouvelles problématiques émergeaient alors avec les premiers échanges sur l'Internet grand public. Ces échanges concernaient non seulement le contenu relatif à la vie privée, mais aussi des œuvres de l'esprit. Je me suis donc, pour commencer, penchée sur les premiers textes législatifs qui visaient à encadrer les échanges de contenus couverts par les droits d'auteur et tout particulièrement sur les directives européennes.

Comme d'autres de mes collègues, je me suis ensuite naturellement intéressée à l'articulation entre les différentes libertés fondamentales dans cet environnement numérique : la liberté d'information et d'expression, la liberté d'entreprendre des opérateurs techniques et, plus généralement, le fonctionnement du marché à l'ère numérique. Des œuvres de l'esprit, j'en suis venue à élargir mes recherches au traitement de données à caractère personnel, puis à la régulation des systèmes algorithmiques. Mes recherches actuelles portent notamment sur la régulation des contenus haineux et des fausses nouvelles diffusés dans les réseaux sociaux, et plus généralement sur le cadre juridique propre à l'activité des plateformes, 
dans le prolongement des travaux que j'avais menés comme membre du Conseil national du numérique. Des réflexions que je poursuis désormais comme personnalité qualifiée au sein de la Commission nationale consultative des droits de l'Homme.

- À vous entendre, il y eut un mouvement de continuité dans votre démarche de chercheure depuis vos premiers travaux jusqu'à vos recherches actuelles, alors qu'on aurait pu penser que l'entrée dans l'ère du Big Data et des réseaux sociaux avait changé la donne, y compris du point de vue $d u$ droit. N'y a-t-il pas eu des défis à relever, ne serait-ce qu'au regard des sources et de la méthodologie?

Pour ma part, je considère qu'il y a eu à la fois de la continuité et de nouveaux défis. De la continuité dans le sens où, aujourd'hui encore, la circulation toujours plus croissante des contenus et des données engendre les mêmes problématiques que celles [qui sont] soulevées par Internet, à ses débuts, notamment en ce qui concerne le type de normes à penser pour s'adapter à la logique du numérique. On fait face aussi aux mêmes interrogations quant aux techniques à concevoir - par exemple, le système algorithmique à même de modérer de façon automatique certains contenus - ou aux régulations devant être mises en place, et, de manière plus générale, à la manière d'adapter le temps et les outils du droit à ceux du numérique.

Défis, ensuite, dans la mesure où il s'agit de se familiariser avec d'autres aspects techniques qui m'étaient étrangers. C'est en ce sens d'ailleurs que mon travail avec la MSH a été bénéfique. Il m'a permis d'échanger davantage avec des informaticiens ou d'autres experts des nouvelles technologies du numérique.

- Dans quel mesure l'environnement de l'Université Paris-Saclay a-t-il lui même été favorable?

J'allais justement y venir, car la création de l'Université Paris-Saclay a indéniablement permis de constituer au fil du temps une communauté scientifique autour de la science de la donnée, au travers notamment de l'Institut de la société numérique (ISN), porté par Nozha Boujemaa. Pour 
mémoire, cet institut avait pour vocation de cartographier la communauté des chercheurs concernés notamment par le numérique, dans l'ensemble des champs disciplinaires, et de favoriser des recherches interdisciplinaires. Mon groupe de recherche d'alors, qui traitait du numérique au prisme de la privacy, en fit naturellement partie. C'est ainsi que nous avons pu nous rapprocher de chercheurs en informatique (notamment de ce qui devait constituer plus tard l'équipe Petrus d'Inria), mais aussi de chercheurs en économie (Grazia Ceccere, par exemple, chercheure au laboratoire Litem - Université d'Évry, IMT Business School) et en droit (ceux du Dante de l'UVSQ et du Cerdi (Centre d'études et de recherche en droit de l'immatériel, de l'Université Paris-Sud).

La dynamique enclenchée par l'ISN devait déboucher sur la création de l'Institut DataIA, également porté par Nozah Boujemaa, et qui m'a été très utile pour lancer, avec Nicolas Anciaux, Directeur de recherche de l'équipe Petrus au centre Inria Saclay Île-de-France, le projet « RGPD et Cloud personnel : de l'Empowerment à la Responsabilité »- projet qui vise à réfléchir aux conditions d'une mise en pouvoir d'agir de l'utilisateur.

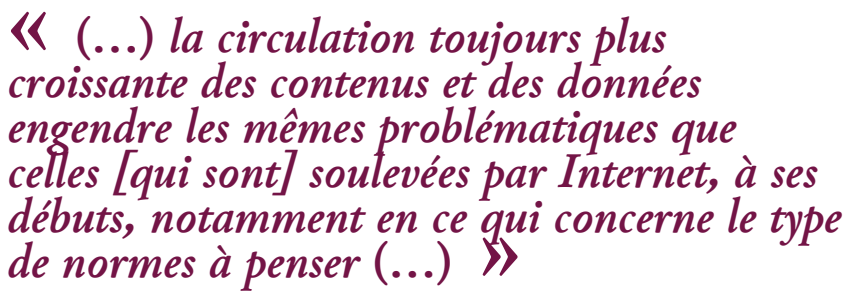

- Cartographie avez-vous dit. C'est précisément ce à quoi s'est employée la MSH pour mettre au jour la communauté des chercheurs en SHS à ParisSaclay...

En effet, et il faut à cet égard saluer le travail accompli par Stefano Bosi, qui en a eu l'initiative en plus de s'y être impliqué personnellement, en n'hésitant pas à prendre son bâton de pèlerin pour aller à la rencontre des responsables d'unités de recherche. Cette cartographie a largement contribué à mettre au jour l'existence, à Paris-Saclay, de SHS plus importantes qu'on pouvait le penser. Tout aussi essentiels sont les événements - workshops et séminaires - qu'il a encouragés : ils ont 
permis aux chercheurs de faire plus ample connaissance, d'identifier des sujets d'étude communs et, ce faisant, de donner chair à la communauté des SHS de Paris-Saclay.

- Quelle a été la valeur ajoutée de la MSH pour vos propres travaux de recherche?

La MSH m'aura permis de poursuivre des recherches sur le thème des algorithmes et des risques de discrimination. Grâce aux moyens mis à disposition, j'ai pu échanger avec d'autres laboratoires de recherche, des industriels et d'autres opérateurs du marché, et ainsi mettre à l'épreuve mes propres hypothèses de travail. Une approche interdisciplinaire est indispensable dans la recherche sur ces enjeux que sont la donnée, l'IA, etc. Seulement, mener des recherches réellement interdisciplinaires, cela demande du temps et de la disponibilité. À défaut de pouvoir allonger la durée de nos journées, la MSH nous simplifie la vie en mettant à dispositions des moyens logistiques.

- Dans quelle mesure la MSH contribue-t-elle à créer de la proximité dans les différents sens du terme où peut l'entendre l'économiste André Torre et plus globalement l'école de la proximité qu'il a contribué à fonder?

Même à l'heure du numérique, les chercheurs ont besoin de se rencontrer. Pour autant, je ne dirai pas que cela passe nécessairement par la création d'un lieu unique. Je crois davantage aux incitations à se montrer ouvert à d'autres approches. Ce qui passe par la valorisation des travaux interdisciplinaires dans le déroulement de la carrière du chercheur. À cet égard, du chemin reste à parcourir, en France du moins. Si mes collègues informaticiens d'Inria commencent à valoriser au sein de leur centre de recherche la contribution à des travaux interdisciplinaires menés au sein de l'Institut DataIA, dans le champ disciplinaire qui est le mien - le droit -, ce n'est pas encore suffisamment le cas. Pourtant, il est clair que les juristes ont besoin d'autres disciplines, à commencer par l'informatique, pour appréhender les enjeux du numérique. Personnellement, je ne maîtrisais pas encore les outils pour prétendre mener seule des recherches dans ce domaine. En disant cela, je ne veux pas dire que l'apport des 
informaticiens se bornerait à de l'assistance. L'enjeu est bien de pouvoir confronter mes hypothèses à d'autres regards, dont celui d'informaticiens en tant qu'ils sont force de propositions sur ces sujets.

\section{《 Au final, la qualité d'un travail interdisciplinaire se mesure à la capacité de faire émerger des notions qui fassent sens dans chacune des disciplines impliquées \}}

- Au-delà des projets de recherche, vous avez eu un rôle actif au sein de la $M S H$, en intégrant le Bureau...

En effet, j'en étais membre, depuis sa création jusqu'en 2018. Je l'étais aussi de celui de l'Institut DataIA. Je me suis donc employée à œuvrer à l'articulation des deux, pour les inciter à porter conjointement des projets dans le domaine des humanités numériques. Un premier travail a consisté à identifier les personnes compétentes sur ces sujets, et plus encore intéressées par la perspective de croiser leurs disciplines. Autant le reconnaitre, constituer une communauté de recherche interdisciplinaire, cela prend du temps, de même que comprendre en quoi le regard, l'expertise de l'autre peut être enrichissant. Il y a encore quelques années - c'est moins vrai aujourd'hui - les chercheurs avaient tendance à rester dans leurs champs disciplinaires respectifs en considérant que l'interdisciplinarité était a priori synonyme de recherche de moindre qualité, car moins spécialisée.

\section{- Comment promouvoir cette interdisciplinarité?}

Toute la difficulté, qu'on rencontre à chaque fois qu'on monte un projet ANR, H 2020 ou autre, c'est de mettre les disciplines à un égal niveau d'intervention, pour produire un travail en commun sans donner l'impression qu'une discipline serait au service des autres. Il est clair que les informaticiens avec lesquels nous travaillons ne sont pas là, j'y reviens, pour régler des problèmes de bugs, mais bien participer à la recherche au même titre que les autres. Pas plus que l'informatique, le juriste n'est là pour s'assurer de la conformité d'une innovation avec la législation en 
vigueur, à commencer par le RGPD. Ce n'est pas ainsi que je conçois l'interdisciplinarité. Il faut que chaque chercheur y trouve son compte et donc commencer par définir un objet à la fois commun et à même de nourrir leurs disciplines respectives.

Au final, la qualité d'un travail interdisciplinaire se mesure à la capacité de faire émerger des notions qui fassent sens dans chacune des disciplines impliquées. Ce qui va bien au-delà de l'exercice consistant à comprendre le sens dans lequel une même notion est entendue dans chacune d'elles. À cet égard, nos recherches disciplinaires ont été fructueuses.

- Pouvez-vous donner un exemple de notion " interdisciplinaire » ayant émergé de ces recherches?

Je pense à la notion d'« agentivité », consistant à considérer l'utilisateur comme un agent du système numérique, à même d'exploiter ses données personnelles pour produire quelque chose qui serve son intérêt, voire l'intérêt collectif. On retrouve là l'enjeu de l'empowerment que j'évoquais. Pour parvenir à cette notion, nous avons chacun puisé dans nos corpus disciplinaires pour converger ensuite progressivement vers un outil de compréhension commun.

- Précisons que vous avez été élue professeure à l'Université Paris 1 PanthéonSorbonne. Gardez-vous des liens avec Paris-Saclay et sa MSH?

Les liens n'ont pas été rompus. Un réseau s'est constitué que je n'hésite pas à solliciter quand une opportunité de projet se présente. Je continue à participer à des contributions communes. Par exemple, actuellement, je poursuis un projet ANR en collaboration avec l'équipe Petrus du Centre Inria Saclay Île-de-France. Malheureusement, les journées ne font que 24 heures. C'est dire s'il faut continuer à créer des facteurs d'incitation. 


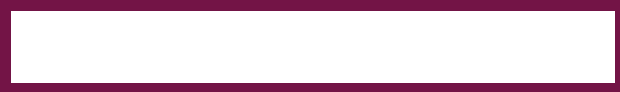

PROGRAMME

\section{DU COLLOQUE}

ANNIVERSAIRE DU

20 NOVEMBRE 2019 


\section{MSH PARIS-SACLAY}

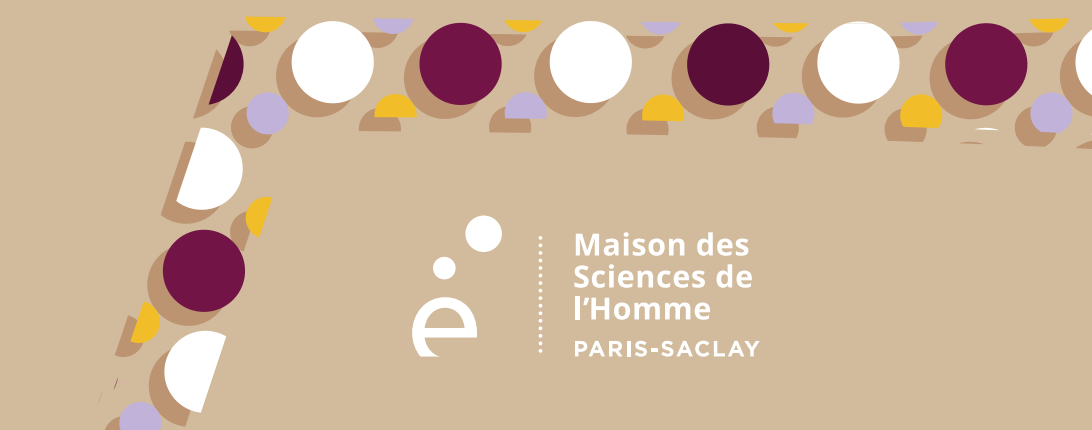

\section{ANS}

d'interdisciplinarité sur un Plateau

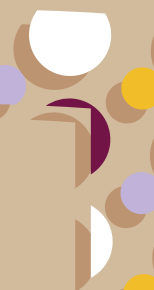

20 NOVEMBRE 2019

HÔTEL DE LAUZUN

ÎLE SAINT-LOUIS, 17 QUAI D'ANJOU 75004 PARIS
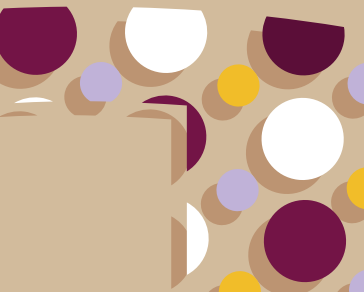

1
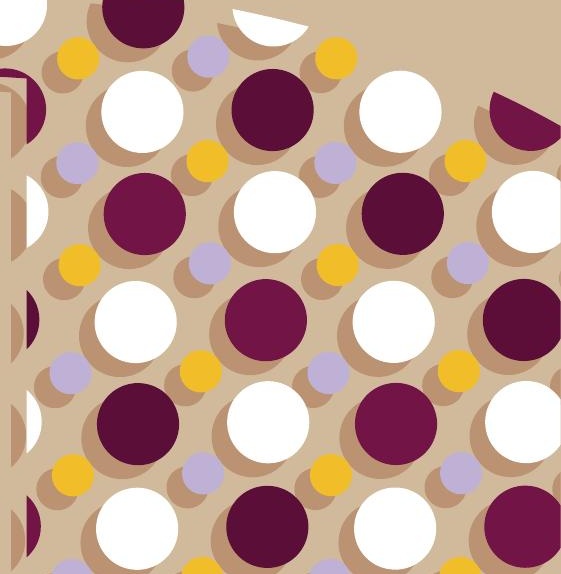

)

1
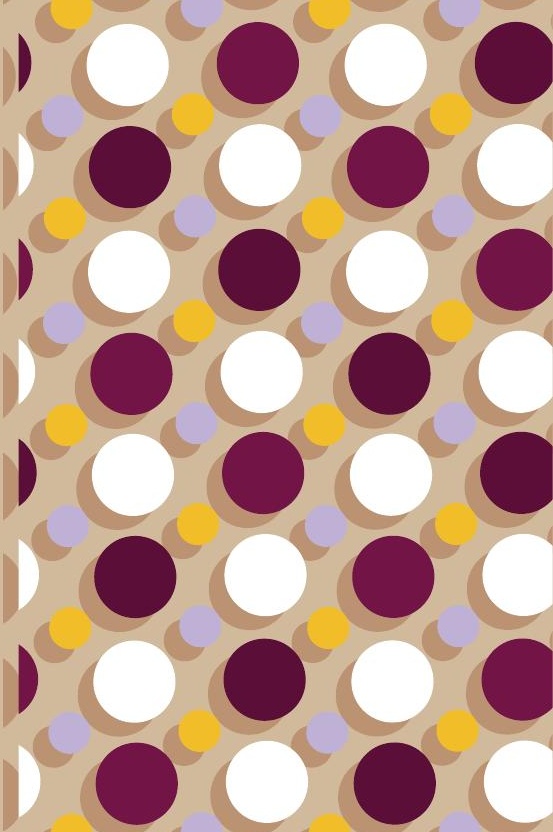

)

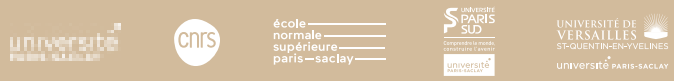
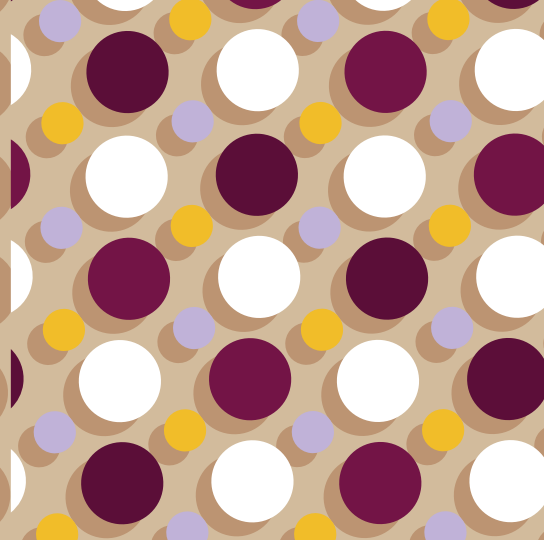


\section{0h - 10h30 : Accueil \& Ouverture}

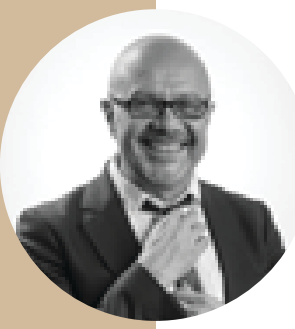

\section{André TORRE}

Directeur de la MSH Paris-Saclay

André Torre est directeur de la MSH Paris-Saclay, président de l'ERSA (European Regional Science Association) et rédacteur en chef de la Revue d'Économie régionale et urbaine. Ses recherches portent principalement sur les relations de proximité et les méthodes de coordination des acteurs territoriaux. Il étudie également les processus de développement et la gouvernance territoriale.

\section{Sylvie RETAILLEAU}

Présidente de l'Université Paris-Saclay

Sylvie Retailleau est Présidente de l'Université ParisSaclay et professeur à l'Université Paris-Sud. Elle a été Doyenne de la faculté des Sciences d'Orsay (20112016), avant de devenir Présidente de l'Université ParisSud (2016-2018).

Ses thèmes de recherche sont centrés sur l'étude théorique de dispositifs pour la nanoélectronique allant du transport au circuit élémentaire avec de nombreux contrats ANR, européens et industriels. Elle a dirigé l'opération «Composants quantiques intégrés pour la nanoélectronique » et a été directrice du PMIPS (Pôle Micro-nanoélectronique de Paris-Sud) du CNFM (Coordination nationale pour la formation en micronanoélectronique).

\section{Hamida DEMIRDACHE}

Directrice adjointe scientifique, InSHS CNRS

Hamida Demirdache est directrice adjointe scientifique de l'InSHS, en charge de la section Sciences du langage et des Maisons des sciences de l'Homme (MSH). Après 
un doctorat en syntaxe formelle réalisé au MIT sous la direction de Noam Chomsky, elle a travaillé sur le terrain pendant cinq ans sur deux langues autochtones de la côte nord-ouest du Pacifique, dans le cadre d'un projet qui explorait la question des limites et de la source de la diversité linguistique. Professeure à l'Université de Nantes, elle a fondé le Laboratoire de Linguistique de Nantes (LLiNG). Elle cherche à intégrer à travers ses travaux les apports de la linguistique théorique et de la linguistique expérimentale en développant simultanément les mêmes thématiques de recherche, que ce soit en syntaxe, en sémantique ou en acquisition du langage, dans une perspective comparative des langues, adultes et enfantines.

\section{1h : Conférence de Valérie Masson-Delmotte}

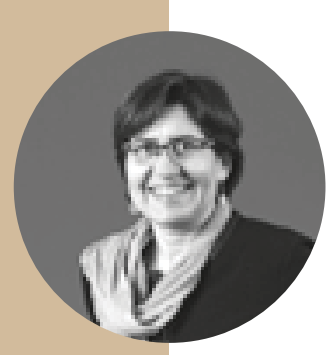

Valérie MASSON-DELMOTTE

Chercheure sénior CEA au LSCE/IPSL

Co-responsable du groupe I du GIEC

Membre du Haut Conseil pour le Climat

\section{Changement climatique, la montée en puissance de l'intégration des connaissances}

Valérie Masson-Delmotte est Directrice de recherches CEA au Laboratoire des sciences du climat et de l'environnement (LSCE) de l'Institut Pierre Simon Laplace à Paris-Saclay, ses recherches portent sur la compréhension du fonctionnement du climat, en particulier par l'étude des climats passés et ont été récompensées par de nombreux prix (Femme Scientifique, 2013 ; Prix Marta T. Muse, 2015 ; Médaille d'argent du CNRS, 2019 ; Médaille Milankovicz, 2020). Elle est élue en 2015 co-présidente du groupe de travail 


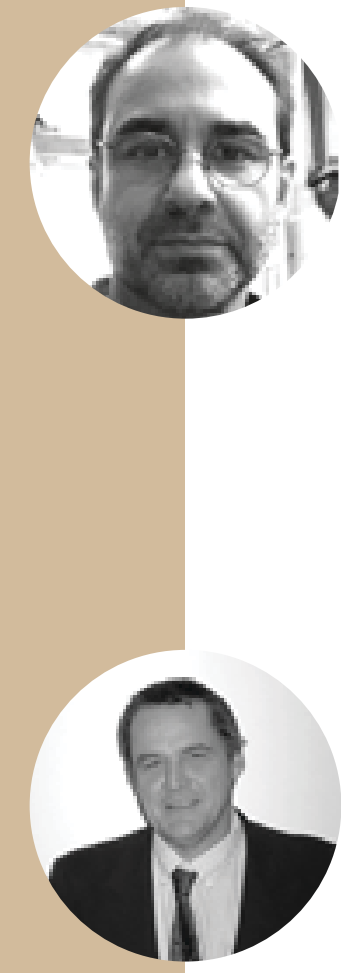

$\mathrm{n}^{\circ} 1 \mathrm{du}$ Groupe d'experts intergouvernemental sur l'évolution du climat (GIEC), et a supervisé les trois rapports spéciaux du GIEC de 2018 et 2019 ainsi que le $6^{\mathrm{e}}$ rapport sur les bases physiques du changement climatique, en préparation pour 2021. Elle est membre du Haut Conseil pour le Climat, créé en 2018. Elle est auteur d'ouvrages à destination des enfants et grand public et très attachée au partage des connaissances liées au changement climatique et aux options pour agir.

\section{Présidée par : Alexis CONSTANTIN}

Vice-Président recherche de l'UVSQ

Alexis Constantin est Agrégé des facultés de droit, Professeur à l'Université de Versailles-Saint-Quentinen-Yvelines et membre du laboratoire de Droit des affaires (Dante) de cette université. Il y enseigne notamment le droit des obligations (contrats et responsabilité civile), le droit des sociétés et le droit commercial international. Après avoir été Directeur de l'Institut d'études judiciaires, il est actuellement VicePrésident de l'UVSQ-Paris-Saclay, en charge de la recherche et du développement scientifique.

\section{Discutant : Jean-Paul VANDERLINDEN Directeur du CEARC/UVSQ}

Jean-Paul Vanderlinden mène actuellement ses recherches sur les enjeux de performativité des sciences sociales dans un contexte de changement climatique. Il travaille à l'interface entre les communautés locales, communauté artistique et communauté scientifique, analysant l'évolution conjointe des discours et des pratiques. Aujourd'hui, ses recherches prennent une tournure transdisciplinaire, les sciences sociales devenant le produit d'une co-construction avec des parties prenantes non scientifiques - utilisateurs des « sciences du climat » artistes et/ou communautés locales. Il est Professeur en sciences économiques en France depuis 2008 et dirige le laboratoire CEARC depuis 2013. Il est, et a été coordinateur et chercheur référant de plusieurs projets internationaux, européens et nationaux. 


\section{4h : Conférence de Laurence Devillers}

\section{Laurence DEVILLERS}

Professeure en Intelligence artificielle à l'Université Paris-Sorbonne IV/Limsi CNRS

\section{Intelligence artificielle $\&$ Robotique sociale et affective : défis pluridisciplinaires et éthiques}

Laurence Devillers, Professeure d'Intelligence artificielle et éthique à Sorbonne Université, dirige l'équipe « Dimensions affectives et sociales dans les interactions parlées » au Limsi (CNRS), elle est membre de la CERNA-Allistène, et auteure de plus de 150 publications scientifiques.

Établir une relation sociale et affective avec les machines n'est plus seulement un rêve d'auteur de science fiction, mais bien une thématique émergente de nombreux chercheurs, dont l'équipe de Laurence Devillers. Ces robots vont habiter nos maisons et également partager avec nous une histoire. Nos capacités d'empathie peuvent conduire à s'illusionner sur les capacités réelles des robots, leur irruption dans nos vies nécessite une réflexion éthique. Si les robots apprennent seuls comme des enfants, il est souhaitable de les programmer de façon éthique by design. À partir de son expertise de chercheure en interaction hommemachine, en informatique émotionnelle et éthique, Laurence Devillers propose de réfléchir à la place des robots dans la société, notamment dans les secteurs de la santé, du bien-être et de l'éducation. 


\section{Présidée par : Pierre-Paul ZALIO Président de l'ENS Paris-Saclay}

Sociologue et acteur du système français d'enseignement supérieur et de recherche, Pierre-Paul Zalio a conduit des recherches sur le patronat et les entrepreneurs avant de prendre la direction de l'ENS Paris-Saclay. Il pilote la reconstruction de cette école par Renzo Piano sur le plateau de Saclay, et est également un des acteurs du projet Paris-Saclay. Parmi ses engagements, il souhaite mettre la culture et la création contemporaine au cœur des dynamiques de recherche et de formation. Après des expériences internationales d'enseignement et de recherche notamment à Shanghai (Hashida), Moscou (MGU) et New-York (Columbia) il a contribué dernièrement à des projets de formation supérieure en Tunisie.

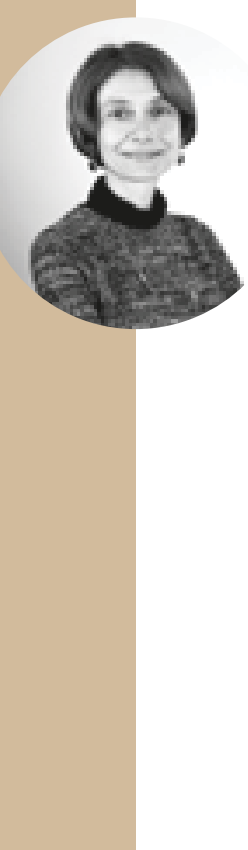

\section{Discutante : Paola TUBARO}

Chargée de recherche CNRS (LRI)

Paola Tubaro a rejoint en 2016 le CNRS, où elle est affectée au Laboratoire de recherche en informatique d'Orsay, après une carrière d'enseignante-chercheure à l'Université de Greenwich (Londres). Économiste de formation, elle mène des recherches interdisciplinaires visant à éclairer des phénomènes socioéconomiques complexes à l'aide de la science des données, de la simulation informatique multiagents et de l'analyse de réseaux sociaux. Ses recherches ont porté sur la vie privée sur Internet, les communautés de santé en ligne, et plus récemment, l'économie des plateformes numériques et les transformations du travail y afférant. Elle s'intéresse également à l'éthique de la recherche sur les réseaux sociaux et aux nouvelles méthodologies liées aux Big Data. Elle enseigne la science des réseaux à l'ENS et à l'Ensae. 


\section{5h30 : Table ronde L'avenir de l'interdisciplinarité en SHS}

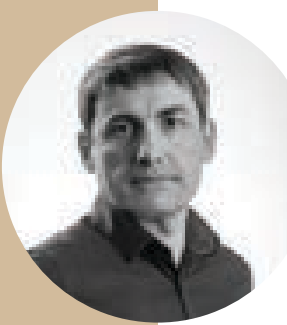

\section{Stefano BOSI}

Vice-Chancelier des Universités de Paris Ancien Directeur de la MSH Paris-Saclay

Stefano Bosi est Professeur d'économie à l'Université d'Évry Val-d'Essonne, ses travaux de recherche portent sur la théorie de l'équilibre général appliquée à divers domaines comme l'économie monétaire et financière, et l'économie de l'environnement. Au plan institutionnel, il s'est fortement impliqué dans la gestion de la science : après avoir été Directeur adjoint scientifique (DAS) des sections 34 (sciences du langage) et 37 (économie et sciences de gestion) du CNRS, il a dirigé l'unité de recherche en économie (EPEE) de l'Université d'Évry, dont il sera aussi le Vice-Président en charge des Relations institutionnelles et présidera le Conseil académique.

En septembre 2016, il succède à Claude Didry à la direction de la MSH Paris-Saclay.

\section{Pascal BULÉON}

Membre du directoire du GIS RnMSH

Directeur de la MRSH Normandie - Caen

Pascal Buléon est Directeur de recherches CNRS, conduit des programmes de recherche sur les questions et développement des territoires, de géographie politique. Il a impulsé et dirige des dispositifs de recherche pluridisciplinaires sur le document numérique. Directeur de la MRSH, Maison de la recherche en Sciences humaine et sociales de Caen. Il préside la Très Grande Infrastructure de recherche françaises PROGEDO pour la culture des données. Il est membre du directoire du Réseau national des MSH (RnMSH). 


\section{Claude DIDRY}

Directeur de recherche CNRS

Ancien Directeur de la MSH Paris-Saclay

Claude Didry est Directeur de recherche CNRS, sociologue, spécialiste de droit du travail. Il a été en charge du chantier de préfiguration de la MSH ParisSaclay qu'il a ensuite dirigée, de sa création jusqu'en août 2016.

Il est, depuis, chercheur au Centre Maurice Halbwachs (CNRS, ENS Ulm, EHESS), après avoir été membre du laboratoire IDHES (antenne de Cachan). Il n'en continue pas moins à cultiver des liens avec la $\mathrm{MSH}$ : les 19-20 septembre 2019, il organisait, avec son soutien, un workshop en l'honneur de l'économiste Robert Salais.

\section{7h : Conférence de Marin Dacos}

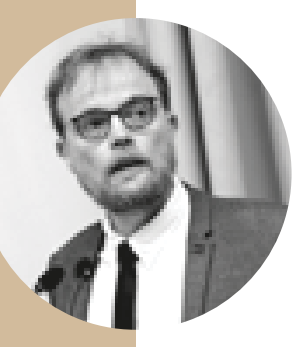

\section{Marin DACOS}

Conseiller scientifique pour la science ouverte au MESRI

\section{La science ouverte : pourquoi, comment, jusqu'où ?}

Marin Dacos est le conseiller pour la Science ouverte auprès du Directeur général de la recherche et de l'innovation au ministère de l'Enseignement supérieur, de la recherche et de l'innovation. Il a reçu la Médaille de l'innovation du CNRS en 2016. Il ouvre depuis plus de vingt ans pour une diffusion des résultats de la recherche en libre accès pour l'ensemble de la société. Il a fondé et dirigé OpenEdition Center, une infrastructure nationale de recherche qui développe OpenEdition, un portail électronique en sciences 


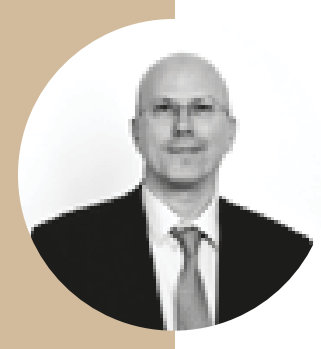

humaines et sociales qui est devenu une référence à l'échelle européenne. Il a publié des livres et articles sur la science ouverte, l'édition électronique scientifique, les humanités numériques.

\section{Présidée par : François-Joseph RUGGIU Directeur de I'InSHS CNRS}

Ancien élève de l'École normale supérieure, agrégé et docteur en histoire, François-Joseph Ruggiu est Professeur à la faculté des Lettres de Sorbonne Université. Spécialiste d'histoire de la famille et des populations en France et en Europe à l'époque moderne, il étudie également l'histoire des sociétés américaines de la période coloniale. Depuis 2010, FrançoisJoseph Ruggiu a occupé de multiples fonctions au sein de l'Institut des sciences humaines et sociales (InSHS) du CNRS en tant que Directeur adjoint scientifique en charge notamment des unités d'histoire, mais aussi des dossiers liés à la valorisation, à la communication, à l'information scientifique et technique ou encore à la politique de site et à la création du Campus Condorcet. Depuis 2017, il est le directeur de l'InSHS du CNRS. 



\section{CRÉDITS}

\section{COUVERTURE ET ILLUSTRATIONS}

(C) Léa Avril pour la MSH Paris-Saclay

\section{BÂTIMENT LAPLACE - ENS PARIS-SALCAY}

(C) Éric Valdenaire pour la MSH Paris-Saclay

\section{COLLOQUE INAUGURAL MSH PARIS-SACLAY}

STEFANO BOSI/ANDRE TORRE

(C) Laurent Zylberman/Graphix-Images

\section{ANTONIO CASILLI}

(C) Hermance Tryai

\section{ANAÏS FLÉCHET}

(C) Sarah Blum

\section{NATHALIE DELPRAT}

(C) Sylvie Pons 



La construction d'un grand pôle scientifique sur le plateau de Saclay est avant tout comprise comme la création d'un fort potentiel de recherche technologique. Pourtant, les Sciences de l'Homme et de la Société ont un rôle majeur à y jouer, par leur volume et par leur place essentielle en termes d'activités et de dispositifs d'innovation.

La MSH Paris-Saclay, créée en 2015, apporte sa contribution à ce défi par son engagement au service des équipes du périmètre saclaysien. Le travail réalisé lui permet d'occuper une place centrale dans la promotion et l'organisation de leurs recherches interdisciplinaires, de développer une position d'interface entre les SHS et de s'ouvrir aux autres disciplines (sciences de la vie, sciences exactes, sciences de l'ingénieur).

Cet ouvrage a pour but de présenter le travail réalisé au cours de ces cinq premières années, à partir d'un bilan des recherches et d'interviews dans lesquels les trois directeurs successifs reviennent sur leur parcours. Dix chercheuses et chercheurs emblématiques des projets passés et en cours apportent également leurs témoignages, afin d'éclairer à la fois la diversité des thèmes de recherche et la variété des résultats obtenus.
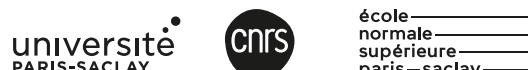

supérieure-

paris-saclay

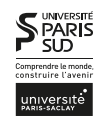

UNIVERSITÉ DE VERSAILLES unIVerSITe் PARIS-SACLAY
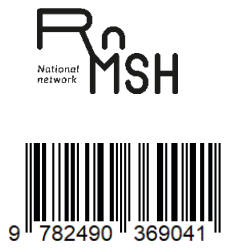